PREPARED For SUbMission to JHEP

\title{
Two-photon exchange corrections to elastic electron-proton scattering at large momentum transfer within the SCET approach
}

\author{
N. Kivel ${ }^{1}$ and $M$. Vanderhaeghen \\ Helmholtz Institut Mainz, Johannes Gutenberg-Universität, D-55099 Mainz, Germany \\ Institut für Kernphysik, Johannes Gutenberg-Universität, D-55099 Mainz, Germany
}

\begin{abstract}
We calculate the two-photon exchange (TPE) corrections in the region where the kinematical variables describing the elastic ep scattering are moderately large momentum scales relative to the soft hadronic scale. For such kinematics we use the QCD factorization approach formulated in the framework of the soft-collinear effective theory (SCET). Such technique allows us to develop a description for the soft-spectator scattering contribution which is found to be important in the region of moderately large scales.

Together with the hard-spectator contribution we present the complete factorization formulas for the TPE amplitudes at the leading power and leading logarithmic accuracy. The momentum region where both photons are hard is described by only one new nonperturbative SCET form factor. It turns out that the same form factor also arises for wide-angle Compton scattering which is also described in the framework of the SCET approach. This allows us to estimate the soft-spectator contribution associated with the hard photons in a model independent way.

The main unknown in our description of the TPE contribution is related with the configuration where one photon is soft. The nonperturbative dynamics in this case is described by two unknown SCET amplitudes. We use a simple model in order to estimate their contribution.

The formalism is then applied to a phenomenological analysis of existing data for the reduced cross section as well as for the transverse and longitudinal polarization observables.
\end{abstract}

\footnotetext{
${ }^{1}$ On leave of absence from St. Petersburg Nuclear Physics Institute, 188350, Gatchina, Russia
} 


\section{Contents}

1 Introduction 1

2 Elastic lepton-nucleon scattering at large $Q^{2} \quad 4$

3 Soft spectator interaction for TPE in SCET $\quad 8$

4 Calculation of the hard coefficient function $\quad 14$

5 Estimate of the soft spectator contribution with $\mathcal{F}_{1}$ from wide-angle $\begin{array}{ll}\text { Compton scattering data } & 18\end{array}$

5.1 QCD factorization for the wide-angle Compton scattering process 18

5.2 Phenomenological analysis of the WACS observables and extraction of the $\begin{array}{ll}\text { ratio } \mathcal{R} & 24\end{array}$

6 Estimate of the amplitudes $g_{1,3}$ in a hadronic model 25

$\begin{array}{lll}7 & \text { Phenomenological analysis } & 31\end{array}$

$\begin{array}{lll}7.1 & \text { Reduced cross section } & 33\end{array}$

$\begin{array}{lll}7.2 & \text { Recoil polarization observables } & 39\end{array}$

8 Conclusions $\quad 41$

$\begin{array}{lll}\text { A Tree level matching } & 44\end{array}$

B Power counting analysis of the SCET operators arising in elastic $e p$ $\begin{array}{ll}\text { scattering } & 45\end{array}$

C Calculation of the one-loop diagrams in Fig.5 52

D Cancellation of the contribution from the hard-collinear regions in the box diagrams $\quad 54$

E Compensation of the QED IR-divergencies in the elastic cross section $\quad 55$

\section{Introduction}

The electromagnetic form factors of the nucleon have been explored extensively during the past 50 years with ever increasing accuracy. The tool to extract the electromagnetic form factors is provided by the one-photon $(1 \gamma)$ exchange approximation to elastic electronnucleon scattering. Precision measurements of the proton electric to magnetic form factor 
ratio at larger $Q^{2}$ using polarization experiments [1-6] have revealed significant discrepancies in recent years with unpolarized experiments using the Rosenbluth technique [7-9], when analyzing both within the $1 \gamma$-exchange framework. This discrepancy between polarized and unpolarized measurements has generated a lot of activity, both theoretical and experimental, over the past decade. The most plausible solution of this problem is the correct calculation of the radiative corrections $(\mathrm{RC})$ to elastic lepton-proton scattering. The well-known calculations in Ref. [10] were performed in the so-called soft photon approximation which allows one to compute the two-photon exchange (TPE) diagrams. This calculation has been recently reconsidered and improved in a series of works [11-17] within a hadronic framework. A more detailed review of the subject can be fond in Refs.[18-20]. However with increasing energy, calculations using hadronic degrees of freedom become less and less reliable. At large energy $s$ and momentum transfer $t \equiv-Q^{2}$ one has to properly take into account the interactions of multiple hard photons with constituents inside the proton. Such dynamics was not considered in the pioneering papers [10] which have been published before the formulation of the underlying microscopic theory of the strong interactions QCD.

A consistent description of the hard and soft QCD dynamics can be carried out using the factorization approach which was applied for the analysis of many exclusive reactions, see e.g. [21-23]. However even this consideration may be sometimes complicated and challenging. The well known example is the description of the nucleon form factors (FFs) at large momentum transfers. For many years a proper theoretical framework for this regime has been the subject of theoretical debates. There are two different points of view on the description of QCD dynamics at large $Q^{2}$. They are related with the different mechanisms of the underlying scattering which we describe as a hard and soft spectator scattering. The hard spectator scattering mechanism was studied long time ago, see e.g. [21-23] and references therein, and results in the well known factorization formula for the FF $F_{1}$. On the other hand the soft spectator scattering has only been estimated using various phenomenological approaches such as light-cone wave functions and QCD sum rules [24-30]. Such estimates show that at moderate values of $Q^{2} \leq 10 \mathrm{GeV}^{2}$ a dominant contribution to the nucleon FFs originates from the soft overlap mechanism.

Recently we suggested the factorization formula for the nucleon FFs [31] which systematically includes both contributions. We used the soft collinear effective theory (SCET) framework in order to describe the factorization of the soft spectator scattering contribution. In this approach the factorization of the soft spectator scattering contribution at moderate values of $Q^{2}$ can be defined in terms of SCET FFs which can be rigorously defined in the intermediate effective theory SCET-I. If the hard-collinear virtualities are relatively small then the further factorization can not be performed and the SCET FFs must be considered as nonperturbative functions.

In the present work we follow the same approach in order to describe the factorization of the short and long distances for the two-photon exchange (TPE) contribution shown on the $l h s$ in Fig.1. This allows us to perform an unambiguous and consistent separation of the different regions in the QED loops in Fig.1 associated with hard and soft configurations of the photons. We restrict our considerations to the region where the Mandelstam variables 
are much larger then the typical QCD scale $s \sim-t \sim-u \gg \Lambda^{2}$, where $\Lambda \sim 0.5 \mathrm{GeV}$ is a soft hadronic scale. In full analogy with the nucleon FFs, the leading power behavior of the

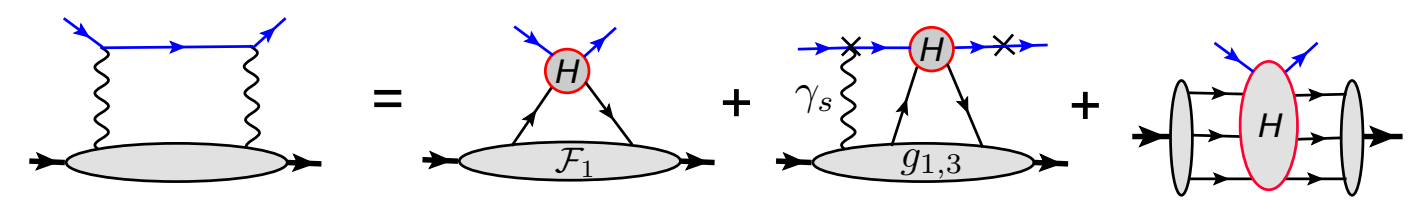

Figure 1. The factorization of the TPE contribution in elastic ep scattering. The crossed box diagram on the lhs is not shown for simplicity. The possible attachments of the soft photon $\gamma_{s}$ to the lepton lines on the rhs are shown by crosses.

TPE amplitudes can be described by two different configurations associated with the soft and hard spectator contributions. Schematically, the structure of the leading contributions is shown in Fig.1. The first two graphs on the rhs describe the soft spectator contribution and the third one corresponds to the hard spectator configuration. The hard subprocesses, which include one or two hard photons are shown as blobs with the symbol $H$. The hard spectator configuration has already been studied in Ref.[32, 33]. The analysis of the soft spectator terms is new. The soft QCD dynamics for the corresponding diagram is described by a SCET FF denoted by $\mathcal{F}_{1}$ and amplitudes $g_{1,3}$ which are defined in SCET-I. At leading order in the QCD coupling, we obtain these new SCET amplitudes which do not appear in the factorization formulas for the nucleon FFs. It turns out that $\mathcal{F}_{1}$ can be fixed from the wide-angle Compton scattering using the universality of its definition in SCET-I. The amplitudes $g_{1,3}$ involve matrix element which cannot be related to known objects, and at present can only be estimated within some model approach. As a first step we consider an estimate using the effective theory with hadronic degrees of freedom. In this way the TPE contribution is completely defined. We then perform phenomenological studies of the TPE effects and compare our results with existing experimental data.

Our paper is organized as follows. Section 2 is devoted to the general properties of elastic ep scattering. We specify notations and kinematics, discuss the general properties of the amplitudes, as well as the structure of the reduced cross section and asymmetries. In section 3 we derive the leading order SCET-I factorization formula for the soft spectator scattering contribution in the TPE amplitudes. In the next section we perform the matching and compute the one-loop, leading order in $\alpha_{s}$ hard coefficient functions. Section 5 is devoted to the extraction of the second unknown $\mathrm{FF} \mathcal{F}_{1}$ from the data for wide-angle Compton scattering. In section 6 we discuss the the SCET amplitudes $g_{1,3}$ which describe the TPE contribution when one of the photons is soft. We use a simple hadronic model in order to estimate this contribution.

In section 7 we use the obtained result for the phenomenological analysis and estimate the effect of the TPE contribution for different observables. The summary of our obtained results is presented in section 8. In Appendices A-E we present more details of some calculations. 


\section{Elastic lepton-nucleon scattering at large $Q^{2}$}

We start by briefly reviewing the main definitions and some results for the process $e(k)+$ $p(p) \rightarrow e\left(k^{\prime}\right)+p\left(p^{\prime}\right)$. In order to describe the electron-nucleon elastic scattering process we introduce the following notations

$$
P=\frac{1}{2}\left(p+p^{\prime}\right), \quad K=\frac{1}{2}\left(k+k^{\prime}\right), q=p^{\prime}-p=k-k^{\prime},
$$

and define the Mandelstam variables

$$
s=(p+k)^{2}, t=q^{2}=-Q^{2}, u=\left(p-k^{\prime}\right)^{2}, \quad \tau=\frac{Q^{2}}{4 m^{2}},
$$

where $m$ is the nucleon mass. For further use, we introduce two more convenient variables

$$
\begin{gathered}
\varepsilon=\left(1+2(1+\tau) \tan ^{2} \frac{\theta}{2}\right)^{-1}=\frac{(s-u)^{2}+t\left(4 m^{2}-t\right)}{(s-u)^{2}-t\left(4 m^{2}-t\right)}, \quad 0<\varepsilon<1, \\
\nu=(K \cdot P)=\frac{s-u}{4},
\end{gathered}
$$

where $\theta$ is the electron Lab scattering angle. One can choose any two independent variables for the description of the physical amplitudes of the process. It is customary to use the variables $Q^{2}$ and $\varepsilon$ for a description of the cross sections and related observables.

Then the general parametrization of the $e p$-scattering amplitude reads [34]

$$
\left.\left\langle p^{\prime}, k^{\prime} \text { out }\right| \text { in } k, p\right\rangle=i(2 \pi)^{4} \delta(p+k-p-k) A_{e p},
$$

where

$$
A_{e p}=\frac{e^{2}}{Q^{2}} \bar{u}\left(k^{\prime}\right) \gamma^{\mu} u(k) \bar{N}\left(p^{\prime}\right)\left[\gamma^{\mu} \tilde{G}_{M}\left(\varepsilon, Q^{2}\right)-\frac{P^{\mu}}{m} \tilde{F}_{2}\left(\varepsilon, Q^{2}\right)+\frac{P^{\mu}}{m^{2}} \not K \tilde{F}_{3}\left(\varepsilon, Q^{2}\right)\right] N(p) .
$$

In the one-photon exchange approximation, this amplitude is given by the well known formula

$$
A_{e p}^{\gamma}=\frac{e^{2}}{Q^{2}} \bar{u}\left(k^{\prime}\right) \gamma^{\mu} u(k) \bar{N}\left(p^{\prime}\right)\left[\gamma^{\mu} G_{M}\left(Q^{2}\right)-\frac{P^{\mu}}{m} F_{2}\left(Q^{2}\right)\right] N(p) .
$$

The following difference can be considered as definition of the TPE corrections arising from the QED next-to-leading corrections

$$
\begin{aligned}
A_{e p}-A_{e p}^{\gamma} & \equiv A_{e p}^{\gamma \gamma}=\frac{e^{2}}{Q^{2}} \bar{u}\left(k^{\prime}\right) \gamma^{\mu} u(k) \\
& \bar{N}\left(p^{\prime}\right)\left[\gamma^{\mu} \delta \tilde{G}_{M}\left(\varepsilon, Q^{2}\right)-\frac{P^{\mu}}{m} \delta \tilde{F}_{2}\left(\varepsilon, Q^{2}\right)+\frac{P^{\mu}}{m^{2}} K_{K} \tilde{F}_{3}\left(\varepsilon, Q^{2}\right)\right] N(p),
\end{aligned}
$$

with

$$
\delta \tilde{G}_{M}\left(\varepsilon, Q^{2}\right)=\tilde{G}_{M}\left(\varepsilon, Q^{2}\right)-G_{M}\left(Q^{2}\right), \delta \tilde{F}_{2}\left(\varepsilon, Q^{2}\right)=\tilde{F}_{2}\left(\varepsilon, Q^{2}\right)-F_{2}\left(Q^{2}\right) .
$$

The amplitudes $\delta \tilde{G}_{M}, \quad \delta \tilde{F}_{2}$ and $\tilde{F}_{3}$ obtain different contributions from all diagrams associated with the QED radiative corrections to elastic ep-scattering. In the present paper 
we only consider the calculation of the leading in $1 / Q^{2}$ corrections arising from the TPE contribution, see Fig.1.

Some useful constraints on the behavior of these amplitudes can be established from the consideration of their analytical properties. For that purpose, following [16] we introduce the functions:

$$
\begin{gathered}
G_{1}\left(\nu, Q^{2}\right)=\delta \tilde{G}_{E}\left(\varepsilon, Q^{2}\right)+\frac{\nu}{m^{2}} \tilde{F}_{3}\left(\varepsilon, Q^{2}\right) \\
G_{2}\left(\nu, Q^{2}\right)=\delta \tilde{G}_{M}\left(\varepsilon, Q^{2}\right)+\frac{\nu}{m^{2}} \tilde{F}_{3}\left(\varepsilon, Q^{2}\right) \\
G_{3}\left(\nu, Q^{2}\right)=\tilde{F}_{3}\left(\varepsilon, Q^{2}\right)
\end{gathered}
$$

where we use the shorthand notation $\varepsilon \equiv \varepsilon\left(\nu, Q^{2}\right)$ and defined $\delta \tilde{G}_{E} \equiv \delta \tilde{G}_{M}-(1+\tau) \delta \tilde{F}_{2}$.

An analysis of $t$-channel helicity amplitudes for the $e p \rightarrow e p$ process shows that in the Regge limit $s \rightarrow \infty, Q^{2} / s \rightarrow 0$, which is equivalent to $\nu \rightarrow \infty, Q^{2} / \nu \rightarrow 0$, the functions $G_{i}$ vanish

$$
\lim _{\nu \rightarrow \infty} G_{i}\left(\nu, Q^{2}\right)=0 .
$$

This higher energy behavior allows one to write down unsubtracted dispersion relations for the amplitudes $G_{i}$ as [16]:

$$
G_{i}\left(\nu, Q^{2}\right)=\int_{\nu_{t h}}^{\infty} d \nu^{\prime} \frac{\operatorname{Im} G_{i}\left(\nu^{\prime}, Q^{2}\right)}{\nu^{\prime}-\nu}-\int_{-\infty}^{-\nu_{t h}} d \nu^{\prime} \frac{\operatorname{Im} G_{i}\left(\nu^{\prime}, Q^{2}\right)}{\nu^{\prime}-\nu} .
$$

The Regge limit can easily be translated into a boundary condition for the practically important variable $\varepsilon$, and corresponds to the limit $\varepsilon \rightarrow 1$ at fixed $Q^{2}$. From Eqs.(2.11 -2.13) one then obtains:

$$
\begin{gathered}
\lim _{\varepsilon \rightarrow 1}\left[\delta \tilde{G}_{E, M}\left(\varepsilon, Q^{2}\right)+\frac{\nu}{m^{2}} \tilde{F}_{3}\left(\varepsilon, Q^{2}\right)\right]=0, \\
\lim _{\varepsilon \rightarrow 1} \tilde{F}_{3}\left(\varepsilon, Q^{2}\right)=0 .
\end{gathered}
$$

Eqs. $(2.16,2.17)$ imply that for certain observables the TPE corrections must vanish in the forward limit.

The analytical expressions for various observables are well known in the literature, see e.g. [34]. For the convenience of the reader we provide some of them here. The unpolarized cross section reads

$$
\frac{d \sigma}{d \Omega_{\mathrm{Lab}}}=\frac{d \sigma_{\mathrm{NS}}}{d \Omega_{\mathrm{Lab}}} \frac{\tau}{\varepsilon(1+\tau)} \sigma_{R}(\varepsilon, Q)
$$

with the structureless part

$$
\frac{d \sigma_{\mathrm{NS}}}{d \Omega_{\mathrm{Lab}}}=\frac{4 \alpha^{2}}{Q^{4}} \cos ^{2} \frac{\theta}{2} \frac{E^{\prime 3}}{E} .
$$

The variables $E$ and $E^{\prime}$ in Eq.(2.19) denote the incoming and outgoing electron Lab energies. The elastic contribution to the reduced cross $\sigma_{R}$ section is given by:

$$
\sigma_{R}^{e l}(\varepsilon, Q)=G_{M}^{2}+\frac{\varepsilon}{\tau} G_{E}^{2}+2 G_{M} \operatorname{Re}\left[\delta \tilde{G}_{M}+\varepsilon \frac{\nu}{m^{2}} \tilde{F}_{3}\right]+2 \frac{\varepsilon}{\tau} G_{E} \operatorname{Re}\left[\delta \tilde{G}_{E}+\frac{\nu}{m^{2}} \tilde{F}_{3}\right] .
$$


The elastic contribution to the polarization observables measured in the recoil polarization experiments reads

$$
\begin{gathered}
\sigma_{R}^{e l} P_{t}=-\sqrt{\frac{2 \varepsilon(1-\varepsilon)}{\tau}}\left\{G_{E} G_{M}+G_{E} \operatorname{Re} \delta \tilde{G}_{M}+G_{M} \operatorname{Re}\left(\delta \tilde{G}_{E}+\frac{\nu}{m^{2}} \tilde{F}_{3}\right)\right\}, \\
\sigma_{R}^{e l} P_{l}=\sqrt{1-\varepsilon^{2}}\left\{G_{M}^{2}+2 G_{M} \operatorname{Re}\left(\delta \tilde{G}_{M}+\frac{\varepsilon}{1+\varepsilon} \frac{\nu}{m^{2}} \tilde{F}_{3}\right)\right\},
\end{gathered}
$$

where $P_{l, t}$ correspond to the recoil proton polarization along or perpendicular to its momentum, respectively. Besides the elastic contributions, shown in Eqs.(2.20-2.22), the observables also include the contribution from the inelastic processes corresponding with the emission of soft photons which provide the cancellation of IR-divergent terms in the elastic next-to-leading QED amplitudes.

Our task is to compute the TPE amplitudes in the limit of large $Q^{2} \gg \Lambda^{2}$ and, if possible, for arbitrary values of $\varepsilon$. However such task includes the analysis of different kinematical regions which can be associated with different underlying QCD dynamics. Therefore we will split the $\varepsilon$-interval into three regions which can be described as follows.

The forward limit $\varepsilon \sim 1$ which can be associated with the Regge limit, in which $s \rightarrow \infty$ and $Q^{2}$ is fixed. In this case as one can conclude from Eqs. $(2.16,2.17)$ and Eq. $(2.20)$ the TPE corrections to the reduced cross section are vanishing.

The second situation is associated with the backward scattering where $s \sim Q^{2} \gg|u| \sim$ $\Lambda^{2}$ and corresponds to the region of small $\varepsilon \ll 1$. From the unpolarized data we expect that in this case the effect of TPE corrections are largest. However, the development of a theoretical approach in a systematic way for this region is a difficult task.

The third region is described by the kinematics where all Mandelstam variables are large and of the same order:

$$
s \sim|u| \sim Q^{2} \gg \Lambda^{2} .
$$

This region can be associated with wide-angle scattering. In this situation one can try to compute the TPE amplitudes by performing an expansion with respect to the large scale $Q^{2}$ with a fixed ratio $Q^{2} / s$. Below we are going to realize this idea using the QCD factorization approach. In this case the values of $\varepsilon$ are restricted to some interval $\varepsilon_{\min }<\varepsilon<\varepsilon_{\max }$ where we define the boundaries $\varepsilon_{\min }$ and $\varepsilon_{\max }$ from the phenomenological criteria that the minimal value of $|u|$ is given by $\left|u_{\text {min }}\right|=2.5 \mathrm{GeV}^{2}$, which guarantees some suppression of subleading power corrections, see Table 2 . The upper boundary can be formally defined by

\begin{tabular}{|c|c|c|c|c|}
\hline$Q^{2}, \mathrm{GeV}^{2}$ & 3 & 4 & 5 & 6 \\
\hline$\varepsilon_{\min }$ & 0.60 & 0.52 & 0.45 & 0.42 \\
\hline
\end{tabular}

the relevance of the Regge dynamics. For simplicity we will not introduce $\varepsilon_{\max }$ assuming an extrapolation to the value $\varepsilon=1$.

The idea to apply the QCD factorization approach for the wide-angle region has already been used in $[32,33]$ where the hard spectator scattering contribution was computed 
at leading order. Because both photons in this case are hard one needs only one-gluon exchange as shown in Fig.2. The nonperturbative input is described by the nucleon distribution amplitudes (DAs), see the details in Refs.[32, 33]. This calculation allows us to
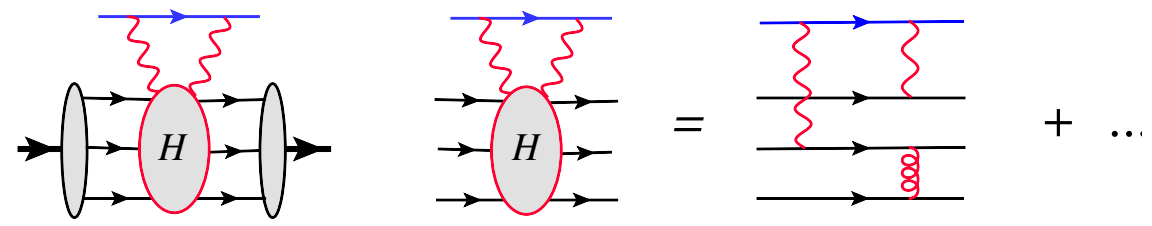

Figure 2. Reduced diagram describing the hard spectator scattering contribution and the leading order diagram with one gluon exchange.

estimate the asymptotic behavior of TPE amplitudes at large $Q^{2}$

$$
\delta G_{M} \sim \frac{\nu}{m^{2}} \delta F_{3} \sim \frac{\Lambda^{4}}{Q^{4}}, \quad \delta F_{2} \sim \frac{\Lambda^{6}}{Q^{6}} .
$$

The behavior of the amplitudes $\delta G_{M}$ and $\delta F_{3}$ is similar to the Dirac FF $F_{1} \sim \Lambda^{4} / Q^{4}$ and can also be described within the collinear factorization approach. The helicity flip amplitudes $\delta F_{2}$ is suppressed by a power $Q^{-2}$ similar to the corresponding Pauli FF $F_{2}$ and can not be described by collinear factorization due to end-point divergencies. Therefore qualitatively, upon neglecting the logarithmic structure, the situation is quite similar to the nucleon FFs.

On the other hand the analysis of the soft spectator scattering for the nucleon FFs shows that these terms are not suppressed by inverse powers of $Q[35,36]$ and therefore can also provide sizable contributions especially in the region of intermediate $Q$ where $Q \Lambda \sim m^{2}$. In Refs.[31, 37] we investigated the soft spectator contribution and suggested the generalization of the factorization which includes both hard and soft spectator terms. Taking into account that the TPE dynamics is quite similar to the FF case one may expect that the same situation is relevant for this case too. Then the soft spectator scattering must also be included into the consideration when calculating the TPE amplitudes.

Such an attempt has been developed in $[38,39]$ where the diagrams in Fig.2 have been evaluated within the framework of the so-called handbag approach [55]. In this model
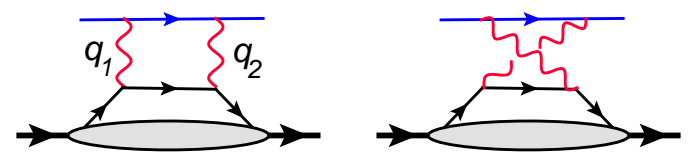

Figure 3. Diagrams describing the soft spectator scattering. Both photons interact with the same quark.

the overlap of the hadronic states are described by the generalized parton distribution (GPD) introduced as matrix element of the light-cone twist-2 matrix element. The GPD which arises here at large $Q$ is considered as a natural generalization of the matrix element which appears in the description of the deeply virtual Compton scattering (DVCS) at small momentum transfer $Q \sim \Lambda$. However in DVCS kinematics, GPDs describe the 
soft dynamics of the system constructed from collinear partons with a small invariant mass (small $Q \sim \Lambda$ ). But at large momentum transfer $(Q \gg \Lambda)$ one is faced with a different situation which may be better associated with the dynamics of jets. In that case the factorization of the soft dynamics is described within the framwork of soft collinear effective theory (SCET) [40-45]. In Ref. [31] we carried out a detailed consideration of the nucleon FFs within the SCET formalism. In the present paper we will show that for the TPE amplitude the situation is quite similar, allowing for a QCD factorization along the same lines.

\section{Soft spectator interaction for TPE in SCET}

The main feature of the SCET factorization is the presence of two large scales: hard $\sim Q^{2}$ and hard-collinear $\sim \Lambda Q$. As a first step, one integrates out the hard modes and passes from the QCD to the effective theory SCET-I. If the value of the hard-collinear scale is not large, for instance, $\Lambda Q \sim m^{2}$ then one can not go on further with the factorization. We shall define such a region of momentum transfers, $Q^{2} \sim 2.5-16 \mathrm{GeV}^{2}$, as intermediate momentum transfer region. At present, this region covers all existing and planned experiments of nucleon FFs.

Our task is to construct a SCET-I factorization for the TPE amplitudes in this region. Such a factorization scheme includes the description of the soft spectator contribution within SCET-I and also, if it is necessary, the hard spectator contributions. These terms can be considered as part of the so-called SCET-II factorization. However, as their factorization scheme is different, e.g. they do not have the Sudakov suppression, they can be considered separately.

Technically the SCET factorization is constructed by matching of QCD diagrams onto appropriate operators in the SCET-I effective theory. The matrix elements of the SCETI operators describe the low scale processes which can not be computed in perturbative QCD. The simple power counting arguments allow one to expect that the leading order SCET-I contribution is given by the diagrams in Fig.2. Obviously, these contributions are leading order with respect to the strong coupling $\alpha_{s}$ but subleading with respect to the electromagnetic constant $\alpha$. Suppose that the incoming and outgoing quarks are hardcollinear. Then in the hard region where all momenta of the particles in the box diagrams are hard $q_{1,2}^{\mu} \sim Q$ this process can be described by perturbative QCD and we obtain the leading order contribution into coefficient function in front of certain SCET-I operators. However, this region is only a part of the full result. For instance, one can also expect that the contribution from the region where only one photon is hard but the second is soft, for instance, $q_{1}^{\mu} \sim \Lambda, q_{2}^{\mu} \sim Q$ can also be relevant. Therefore the SCET factorization must properly take into account all possible dominant regions associated with factorization of the hard modes.

In the following we use two light-cone vectors $n=(1,0,0,-1)$ and $\bar{n}=(1,0,0,1)$ and choose the Breit frame where the initial and final proton are collinear to the $z$-axis. Then 
the light-cone expansion of the external momenta read

$$
\begin{gathered}
q=Q \frac{n}{2}-Q \frac{\bar{n}}{2}, \quad p \simeq Q \frac{\bar{n}}{2}, \quad p^{\prime} \simeq Q \frac{n}{2}, \\
k \simeq \frac{\bar{z}}{z} Q \frac{\bar{n}}{2}+\frac{1}{z} Q \frac{n}{2}+k_{\perp}, \quad k^{\prime} \simeq \frac{1}{z} Q \frac{\bar{n}}{2}+\frac{\bar{z}}{z} Q \frac{n}{2}+k_{\perp},
\end{gathered}
$$

where we introduced for convenience the dimensionless parameter $z=-t / s$, with $\bar{z} \equiv 1-z$. The Breit system is convenient for the description of the large $Q^{2}$ behavior of the nucleon FFs and it is natural to use it for the consideration of the TPE contributions too.

Let us briefly describe the SCET notations used in our paper. We will use the SCET formulation in coordinate space developed in [44]. For the SCET fields we use the following notations. The fields $\xi_{n}, A_{\mu}^{(n)}$ and $\xi_{\bar{n}}, A_{\mu}^{(\bar{n})}$ denote hard-collinear quark and gluon fields associated with momentum $p^{\prime}$ and $p$, respectively, see Eq.(3.1). As usually, the hardcollinear quark fields satisfy

$$
\not h \xi_{n}=0, \not h \xi_{\bar{n}}=0 .
$$

The fields $q$ and $A_{\mu}^{(s)}$ denote the soft quarks and gluons which also enter in the SCET Lagrangian. These fields describe particles with soft momenta $k_{\mu} \sim \Lambda .{ }^{1}$ We also use the convenient notation for the gauge invariant combinations often appearing in SCET such as

$$
\chi_{n}(\lambda \bar{n}) \equiv W_{n}(\lambda \bar{n}) \xi_{n}(\lambda \bar{n})
$$

where the hard-collinear gluon Wilson line (WL) reads :

$$
W_{n}(z)=\mathrm{P} \exp \left\{i g \int_{-\infty}^{0} d s \bar{n} \cdot A^{(n)}(z+s \bar{n})\right\} .
$$

In the QED sector we also split the fields according to the same SCET prescription. Let us notice that the pure QED radiative corrections (electron vertex and self-energy) can be computed exactly and for such calculations such representations are not required. However in case of the TPE corrections, the hard and soft photons correspond with different dynamics, making the SCET representation useful.

In the wide-angle kinematics we have four directions with a large energy flow. It is therefore useful to introduce two more auxiliary light-cone vectors associated with the lepton momenta: $k$ and $k^{\prime}$

$$
\bar{v}^{\mu}=\frac{2 k^{\mu}}{Q}, \quad v^{\mu}=\frac{2 k^{\prime \mu}}{Q},(\bar{v} \cdot v)=2
$$

Then, an arbitrary vector can be decomposed as

$$
V^{\mu}=(V \cdot \bar{v}) \frac{v^{\mu}}{2}+(V \cdot v) \frac{\bar{v}^{\mu}}{2}+V_{T}
$$

\footnotetext{
${ }^{1}$ In Ref.[44] these modes are introduced as ultra-soft. In this work we use the different terminology suggested in Refs.[46, 47] .
} 
where we denoted the transverse components with respect to the $v, \bar{v}$ basis by $V_{T}$ with subscript $T$, in order to distinguish them from the transverse components in the $n, \bar{n}$ basis which we denote as $V_{\perp}$. Using the vectors $\bar{v}, v$ we introduce the hard-collinear lepton fields $\zeta_{\bar{v}}$ and $\zeta_{v}$, satisfying :

$$
\not \zeta_{v}=0, \not \zeta_{\bar{v}}=0,
$$

and the soft photon field by $B_{\mu}^{(s)}$. This is a minimal set of the auxiliary fields in QED which we need for our considerations. We do not introduce the collinear photon fields because they are not required for a description of the TPE amplitudes. We always use the Feynman gauge for the gluon and photon fields.

We start our consideration from the one-photon exchange. In the following, we will only consider the soft-spectator scattering contribution. The soft-spectator scattering for nucleon FFs has been studied in [31,37]. We briefly repeat the results relevant for our calculations. To the leading order, the SCET expression for the amplitude $A_{e p}$ can be written as

$$
A_{e p}^{\gamma} \simeq \frac{4 \pi \alpha}{Q^{2}} \bar{u}\left(k^{\prime}\right) \gamma^{\mu} u(k) C_{+}(Q, \mu)\left\langle p^{\prime}\left|O_{+}^{\mu}(0)\right| p\right\rangle_{\mathrm{SCET}}
$$

where the leading power SCET-I operator reads

$$
O_{+}^{\mu}(0)=\sum_{q} e_{q}\left\{\bar{\chi}_{n}(0) \gamma_{\perp}^{\mu} \chi_{\bar{n}}(0)+\bar{\chi}_{\bar{n}}(0) \gamma_{\perp}^{\mu} \chi_{n}(0)\right\}
$$

and $C_{+}^{\mu \nu}$ denotes the hard coefficient function. Let us note that the operator in Eq.(3.10) has an additional term compared to the operator considered in [31]. This term given by $\bar{\chi}_{\bar{n}} \gamma_{\perp}^{\mu} \chi_{n}$ in Eq.(3.10) can be associated with the scattering of the hard photon on the antiquark. Such contribution describes the hadronization of the hard-collinear antiquark into proton. Because the proton is dominated by three quark state such term is suppressed by the powers of the hard-collinear scale in the transition from SCET-I to SCET-II. If the hard-collinear scale is not large and we restrict our consideration only to the SCET-I theory, we can not neglect such operators. The relative sign between the two terms in Eq.(3.10) is dictated by $C$-parity. The subscript SCET in Eq.(3.9) means that the matrix element has to be computed with the SCET Lagrangian $\mathcal{L}_{\mathrm{SCET}}=\mathcal{L}_{\mathrm{SCET}}^{(n)}+\mathcal{L}_{\mathrm{SCET}}^{(\bar{n})}+\mathcal{L}_{\text {soft }}$ which represent by the sum of Lagrangians describing each collinear sector. The explicit expressions for $\mathcal{L}_{\text {SCET }}^{(n)}$ with the fields defined in position space can be found in Refs. [44, 45]. The SCET matrix element in Eq.(3.9) describes the interaction of the jets of the hard-collinear particles represented by $\chi_{n, \bar{n}}$ with the soft background described by the soft quark and gluons in the SCET Lagrangians. This dynamics can also be understood as a soft-overlap of the initial and final hadronic states. The parametrization of this matrix element reads

$$
\left\langle p^{\prime}\left|O_{+}^{\mu}(0)\right| p\right\rangle_{\mathrm{SCET}}=\bar{N}\left(p^{\prime}\right) \frac{\not h_{h}}{4} \gamma_{\perp}^{\mu} N(p) f_{1}(Q, \mu),
$$

where $f_{1}$ is the SCET FF. By construction it depends on the hard-collinear scale, referred to by the argument $Q$, whereas the second argument $\mu$ denotes the renormalization scale. Performing the matching it is convenient to put $\mu=Q$ and then to evolve it down to 
the values of the hard-collinear scale $\mu \sim \sqrt{\Lambda Q}$. The evolution is described by the renormalization of the SCET operator, see e.g. [48]. In what follow we suggest to use simple notation

$$
f_{1}(Q, \mu=Q) \equiv f_{1}(Q) .
$$

Taking into account that the tree level coefficient function reads

$$
C_{+}(Q, \mu=Q)=1+\mathcal{O}\left(\alpha_{s}\right)
$$

one obtains

$$
A_{e p}^{\gamma}=\frac{4 \pi \alpha}{Q^{2}} \bar{u}\left(k^{\prime}\right) \gamma^{\mu} u(k) \quad \bar{N}\left(p^{\prime}\right) \frac{\not h \hbar}{4} \gamma_{\perp}^{\mu} N(p) f_{1}(Q) .
$$

Comparing this with the $A_{\gamma}$ in Eq.(2.7) one finds (see details in Appendix A)

$$
G_{M}\left(Q^{2}\right)=f_{1}(Q), \quad F_{2}\left(Q^{2}\right)=\frac{4 m^{2}}{Q^{2}} f_{1}(Q) .
$$

The Pauli FF $F_{2}$ is suppressed by the factor $m^{2} / Q^{2}$ due to one unit of helicity flip. Moreover, this expression is not complete because it also includes the contribution from the subleading operator, see e.g.[31], which we do not consider now for simplicity. ${ }^{2}$

The generalization of this approach to next-to-leading order in QED can be done along the same lines. It is convenient to include the soft-photon field into the SCET Lagrangian similar to the soft-gluon field and perform the expansion at the last step. Then for the elastic amplitude we can write

$$
A_{e p} \simeq\left\langle p^{\prime}, k^{\prime}\left|T\left\{\tilde{C}_{+}^{\mu \nu} * O_{+}^{\mu} O_{e}^{\nu}+\tilde{C}_{-}^{\mu \nu} * O_{-}^{\mu} O_{e}^{\nu}\right\}\right| k, p\right\rangle_{\mathrm{SCET}},
$$

where the hard coefficient functions $C_{ \pm}^{\mu \nu}$ describe the hard subprocesses, the asterisk denotes the convolution integrals in position space and the SCET operators $O_{ \pm}^{\mu}$ describe the dynamics of hard-collinear and soft particles and read:

$$
\begin{aligned}
O_{ \pm}^{\mu} \equiv O_{ \pm}^{\mu}\left(\lambda_{1}, \lambda_{2}\right)= & \sum_{\text {flavors }}\left\{\bar{\chi}_{n}\left(\lambda_{1} \bar{n}\right) \gamma_{\perp}^{\mu} \chi_{\bar{n}}\left(\lambda_{2} n\right) \pm \bar{\chi}_{\bar{n}}\left(\lambda_{2} n\right) \gamma_{\perp}^{\mu} \chi_{n}\left(\lambda_{1} \bar{n}\right)\right\} \\
& O_{e}^{\nu} \equiv O_{e}^{\nu}\left(\eta_{1}, \eta_{2}\right)=\bar{\chi}_{v}\left(\eta_{1} \bar{v}\right) \gamma^{\nu} \chi_{\bar{v}}\left(\eta_{2} v\right),
\end{aligned}
$$

where we introduced

$$
\bar{\chi}_{v}=\bar{\zeta}_{v} W_{v}, \chi_{\bar{v}}=W_{\bar{v}}^{\dagger} \zeta_{\bar{v}}
$$

with the hard-collinear photon WLs

$$
\begin{aligned}
& W_{\bar{v}}(z)=\mathrm{P} \exp \left\{-i e \int_{-\infty}^{0} d t v \cdot B_{h c}^{(\bar{v})}(z+v t)\right\} \\
& W_{v}(z)=\mathrm{P} \exp \left\{-i e \int_{-\infty}^{0} d t \bar{v} \cdot B_{h c}^{(v)}(z+\bar{v} t)\right\} .
\end{aligned}
$$

\footnotetext{
${ }^{2}$ In [31] the kinematical power corrections to the SCET FF $f_{1}$ were missed. The complete leading power contribution is given by the sum $F_{2}=4 m^{2} f_{1} / Q^{2}+m^{2} C_{B} * f_{2} / Q^{2}$ where $C_{B}$ is the subleading coefficient functions, in the notation of Ref. [31].
} 
Taking into account that these WLs are not required for the TPE calculation we can skip them assuming

$$
O_{\zeta}^{\nu}\left(\eta_{1}, \eta_{2}\right) \simeq \bar{\zeta}_{v}\left(\eta_{1} \bar{v}\right) \gamma^{\nu} \zeta_{\bar{v}}\left(\eta_{2} v\right)
$$

We also assume a similar simplification for the operators $O_{ \pm}^{\mu}$. This implies that we reduce our considerations only to TPE diagrams in Fig. $2 .{ }^{3}$ The operators $O_{ \pm}^{\mu}$ represent the operators which arise at leading power and leading order in $\alpha_{s}$. The other operators are subleading and provide only subleading contributions according to SCET power counting or are subleading in $\alpha_{s}$. A more detailed consideration of this observation is provided in Appendix B.

The $C$-even operator $O_{-}^{\mu}$ can appear only due to the hard two-photon exchange. The corresponding hard coefficient function $\tilde{C}_{-}^{\mu \nu}$ can be associated with the hard region in the TPE diagrams in Fig.2, i.e. $\tilde{C}_{-}^{\mu \nu} \sim \mathcal{O}\left(\alpha^{2}\right)$. Hence computing the matrix element $\left\langle O_{-}^{\mu} O_{e}^{\nu}\right\rangle$ we can neglect the soft photons in the SCET Lagrangian because they provide only higher order $\mathcal{O}\left(\alpha^{3}\right)$ contributions. Therefore the leptonic matrix element can be factorized as :

$$
\left\langle p^{\prime}, k^{\prime}\left|T\left\{\tilde{C}_{-}^{\mu \nu} * O_{-}^{\mu} O_{e}^{\nu}\right\}\right| k, p\right\rangle_{\mathrm{SCET}}=\left\langle k^{\prime}\left|O_{e}^{\nu}\right| k\right\rangle_{\mathrm{SCET}} * \tilde{C}_{-}^{\mu \nu} *\left\langle p^{\prime}\left|O_{-}^{\mu}\right| p\right\rangle_{\mathrm{SCET}}
$$

The hadronic matrix element gives the new SCET FF which we define as follows :

$$
\left\langle p^{\prime}\left|O_{-}^{\mu}\left(\lambda_{1}, \lambda_{2}\right)\right| p\right\rangle_{\mathrm{SCET}}=e^{i\left(p^{\prime} \bar{n}\right) \lambda_{1}-i(p n) \lambda_{2}} \bar{N}\left(p^{\prime}\right) \frac{\not h \pitchfork}{4} 4 \gamma_{\perp}^{\mu} N(p) \mathcal{F}_{1}(Q, \mu) .
$$

The dependence on $Q$ and $\mu$ has to be understood in the same way as in case FF $f_{1}$ defined in Eq.(3.11). Substituting this into Eq.(3.23) yields

$$
\begin{aligned}
&\left\langle p^{\prime}, k^{\prime}\left|T\left\{\tilde{C}_{-}^{\mu \nu} * O_{-}^{\mu} O_{e}^{\nu}\right\}\right| k, p\right\rangle_{\mathrm{SCET}}= \\
& \frac{e^{2}}{Q^{2}} \bar{u}_{v} \gamma^{\nu} u_{\bar{v}} \bar{N}\left(p^{\prime}\right) \frac{\text { hh }_{h}}{4} \gamma_{\perp}^{\mu} N(p) C_{-}^{\mu \nu}\left(z, Q^{2}, \mu_{F}, \mu\right) \mathcal{F}_{1}(Q, \mu),
\end{aligned}
$$

where $C_{-}^{\mu \nu}$ is the Fourier transformation of the coefficient function $\tilde{C}_{-}^{\mu \nu}$ defined in position space, $u_{v, \bar{v}}$ denote large components of the electron spinors $\psi u_{v}=0, \not{\psi} u_{\bar{v}}=0$. Notice that such approximations is exact in the limit of massless electrons, $m_{e}=0$. The coefficient function $C_{-}^{\mu \nu}$ depends on the two different factorization scales $\mu_{F}$ and $\mu$. The factorization scale $\mu_{F}$ describes the factorization of the QED loop and is closely associated with the virtualities of the photons. The factorization scale $\mu$ describes the factorization for QCD degrees of freedom. We will set $\mu=Q$ and assume $\mathcal{F}_{1}(Q, \mu=Q) \equiv \mathcal{F}_{1}(Q)$. The contribution given in Eq.(3.25) corresponds to the first graph on rhs of Fig.1.

Consider now the first term in Eq.(3.16). This contribution must be computed taking into account the presence of the soft photons in the SCET Lagrangians. The situation in the leptonic sector can be simplified if we factorize the soft photons using the fields redefinition, see e.g.[49]

$$
\bar{\zeta}_{v}\left(\eta_{1} \bar{v}\right)=\bar{\zeta}_{v}^{(0)}\left(\eta_{1} \bar{v}\right) Y_{v}^{\dagger}(0), \quad \zeta_{\bar{v}}\left(\eta_{2} v\right)=S_{\bar{v}}(0) \zeta_{\bar{v}}^{(0)}\left(\eta_{2} v\right),
$$

\footnotetext{
${ }^{3}$ Let us note that each individual box diagram has the regions associated with collinear photons. However, in the sum these contributions cancel due to gauge invariance.
} 
where the soft photon WLs reads

$$
\begin{gathered}
Y_{v}^{\dagger}(0)=\mathrm{P} \exp \left\{-i e \int_{0}^{\infty} d t v \cdot B^{(s)}(t v)\right\} \\
S_{\bar{v}}(0)=\mathrm{P} \exp \left\{-i e \int_{-\infty}^{0} d t \bar{v} \cdot B^{(s)}(t \bar{v})\right\} .
\end{gathered}
$$

The new electron fields $\zeta_{v, \bar{v}}^{(0)}$ do not interact with the soft photons anymore and therefore the leptonic matrix element is factorized

$$
\begin{aligned}
\left\langle p^{\prime}, k^{\prime}\left|T\left\{\tilde{C}_{+}^{\mu \nu} * O_{+}^{\mu} O_{e}^{\nu}\right\}\right| k, p\right\rangle_{\mathrm{SCET}} \simeq \tilde{C}_{+}^{\mu \nu} *\left\langle p^{\prime}\left|T\left\{O_{+}^{\mu}\left(\lambda_{1}, \lambda_{2}\right) Y_{v}^{\dagger}(0) S_{\bar{v}}(0)\right\}\right| p\right\rangle_{\mathrm{SCET}} \\
\\
\times\left\langle k^{\prime}\left|\left\{\bar{\zeta}_{v}^{(0)}\left(\eta_{1} \bar{v}\right) \gamma^{\nu} \zeta_{\bar{v}}^{(0)}\left(\eta_{2} v\right)\right\}\right| k\right\rangle_{\mathrm{SCET}},
\end{aligned}
$$

and can be easily computed ${ }^{4}$

$$
\left\langle k^{\prime}\left|\left\{\bar{\zeta}_{v}^{(0)}\left(\eta_{1} \bar{v}\right) \gamma^{\nu} \zeta_{\bar{v}}^{(0)}\left(\eta_{2} v\right)\right\}\right| k\right\rangle_{\mathrm{SCET}}=e^{i \eta_{1}\left(\bar{v} \cdot k^{\prime}\right)-i \eta_{2}(v \cdot k)} \bar{u}_{v} \gamma^{\nu} u_{\bar{v}}
$$

The hadronic matrix element can be considered as a generalization of the leading order matrix element defined in Eq.(3.11) in the presence of the soft photons created by lepton source. Therefore we can write

$$
\begin{aligned}
\left\langle p^{\prime}\right| T\{ & \left.O_{+}^{\mu}\left(\lambda_{1}, \lambda_{2}\right) Y_{v}^{\dagger}(0) S_{\bar{v}}(0)\right\}|p\rangle_{\mathrm{SCET}}=e^{i\left(p^{\prime} \bar{n}\right) \lambda_{1}-i(p n) \lambda_{2}} \bar{N}\left(p^{\prime}\right) \frac{\not h t h}{4} \gamma_{\perp}^{\sigma} N(p) \\
& \times\left\{g_{\perp}^{\mu \sigma}\left[f_{1}(Q)+\frac{\alpha}{\pi} g_{1}(z, Q)\right]+v_{\perp}^{\sigma} v_{\perp}^{\mu} \frac{\alpha}{\pi} \frac{4(z-2)}{z^{2}} g_{3}(z, Q)\right\}
\end{aligned}
$$

where the SCET amplitudes $g_{1,3}$ represent the next-to-leading QED correction arising from the interaction of the soft photons with the hard-collinear and soft spectator quarks. The coefficient $4(z-2) / z^{2}$ is introduced for convenience. These functions also depend on the factorization scales $\mu_{F}$ and $\mu$ which we do not show in Eq.(3.31) for simplicity. Substituting (3.30) and (3.31) into (3.29) we obtain

$$
\begin{aligned}
\left\langle p^{\prime}, k^{\prime}\right| T\left\{\tilde{C}_{+}^{\mu \nu}\right. & \left.* O_{+}^{\mu} O_{e}^{\nu}\right\}|k, p\rangle_{\mathrm{SCET}}=C_{+}^{\mu \nu} \bar{u}_{v} \gamma^{\nu} u_{\bar{v}} \bar{N}\left(p^{\prime}\right) \frac{\not h \hbar}{4} \gamma_{\perp}^{\sigma} N(p) \\
& \times\left\{g_{\perp}^{\mu \sigma}\left[f_{1}(Q)+\frac{\alpha}{\pi} g_{1}(z, Q)\right]+v_{\perp}^{\sigma} v_{\perp}^{\mu} \frac{\alpha}{\pi} \frac{4(z-2)}{z^{2}} g_{3}(z, Q)\right\} .
\end{aligned}
$$

The contributions with $g_{1,3}$ in Eq.(3.32) corresponds to the second graph on rhs of Fig.1. The coefficient function $C_{+}^{\mu \nu}$ is fixed by the one-photon approximation. Using Eqs.(3.9) one obtains

$$
C_{+}^{\mu \nu}=g^{\mu \nu} \frac{4 \pi \alpha}{Q^{2}} C_{+}(Q, \mu)=g^{\mu \nu} \frac{4 \pi \alpha}{Q^{2}}+\mathcal{O}\left(\alpha_{s}\right)+\mathcal{O}\left(\alpha^{2}\right) .
$$

The terms of order $\mathcal{O}\left(\alpha^{2}\right)$ includes pure QED corrections like electron and photon self energies, photon-electron vertex corrections and also hadron vertex and self-energy which

\footnotetext{
${ }^{4}$ Here we do not describe the formal details related to transition from SCET-I to SCET-II in the leptonic sector.
} 
we will not recompute in this work. Using Eq.(3.33), the TPE contribution to the amplitude $A_{e p}^{\gamma \gamma}$ can be written as

$$
\begin{gathered}
A_{e p}^{2 \gamma}=\frac{4 \pi \alpha}{Q^{2}} \bar{u}_{v} \gamma^{\nu} u_{\bar{v}} \bar{N}\left(p^{\prime}\right) \frac{\not \hbar \hbar}{4} \gamma_{\perp}^{\sigma} N(p)\left\{g_{\perp}^{\nu \sigma}\left[f_{1}(Q)+\frac{\alpha}{\pi} g_{1}(z, Q)\right]+\frac{1}{s} K_{\perp}^{\sigma} P^{\nu} \frac{\alpha}{\pi} g_{3}(z, Q)\right\} \\
+\bar{u}_{v} \gamma^{\nu} u_{\bar{v}} \bar{N}\left(p^{\prime}\right) \frac{\not h \hbar}{4} \gamma_{\perp}^{\mu} N(p) C_{-}^{\mu \nu}\left(z, Q^{2}\right) \mathcal{F}_{1}(Q),
\end{gathered}
$$

where we used that

$$
v_{\perp}^{\sigma} v_{\perp}^{\mu} \frac{\alpha}{\pi} \frac{4(z-2)}{z^{2}} \bar{u}_{v} \gamma^{\mu} u_{\bar{v}} \bar{N}\left(p^{\prime}\right) \frac{\not h \hbar}{4} \gamma_{\perp}^{\sigma} N(p)=\frac{1}{s} K_{\perp}^{\sigma} P^{\mu} \bar{u}_{v} \gamma^{\mu} u_{\bar{v}} \bar{N}\left(p^{\prime}\right) \frac{\not h \pitchfork}{4} \gamma_{\perp}^{\sigma} N(p) .
$$

The expression in (3.34) is our final expression for the soft spectator scattering contribution describing elastic ep-scattering amplitudes with the TPE corrections as in Fig.2. In order to use this formula in phenomenological applications, we have to compute the hard coefficient function $C_{-}^{\mu \nu}$ and estimate the SCET FFs which enter in Eq.(3.34).

\section{Calculation of the hard coefficient function}

The calculation of the hard coefficient function $C_{-}^{\mu \nu}$ can be done by matching of the box diagram in Fig.4. In order to perform the matching we consider quark instead of proton

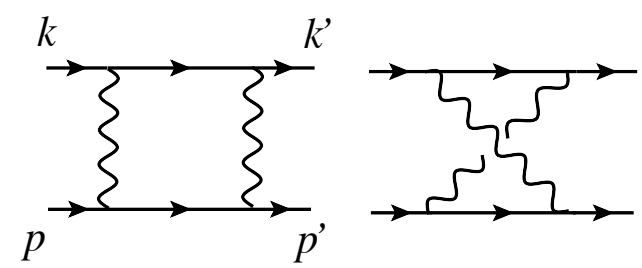

Figure 4. Two-photon exchange diagrams with external momenta required for calculation of the hard coefficient function $C_{-}^{\mu \nu}$

states. ${ }^{5}$. The expressions for the diagrams with massless quarks are well known, see e.g. $[38,39,50]$ and read

$$
A_{e q}^{2 \gamma}=\frac{4 \pi \alpha}{Q^{2}} \bar{u}_{v} \gamma_{\mu} u_{\bar{v}} \bar{q}_{n}\left\{\gamma^{\mu} \frac{\alpha}{\pi} e_{q}^{2} \tilde{f}_{1}+\frac{1}{s} P^{\nu} \not K \frac{\alpha}{\pi} e_{q}^{2} \tilde{f}_{3}\right\} q_{\bar{n}}+\bar{v}_{n}\{\ldots\} v_{\bar{n}},
$$

where and $q_{n, \bar{n}}$ and $v_{n, \bar{n}}$ denote the SCET spinors for the quark and antiquark, respectively. The amplitudes $\tilde{f}_{i}$ read (recalling $z=-t / s$ )

$$
\begin{gathered}
\operatorname{Re} \tilde{f}_{1}=\ln \bar{z} \ln \frac{s}{\lambda^{2}}+\frac{1}{2} \ln ^{2} \bar{z}-\frac{1}{4} \frac{z \ln ^{2} z}{1-z}-\frac{z}{4} \ln ^{2} \frac{\bar{z}}{z}-\frac{1}{2} \ln \bar{z}-\frac{z \pi^{2}}{4}+\frac{\pi^{2}}{2}, \\
\operatorname{Re} \tilde{f}_{3}=\frac{2-z}{2 \bar{z}^{2}} \ln ^{2} z-\frac{2-z}{2} \ln ^{2} \frac{\bar{z}}{z}+\frac{1}{\bar{z}} \ln z+\ln \frac{\bar{z}}{z}-\frac{2-z}{2} \pi^{2},
\end{gathered}
$$

\footnotetext{
${ }^{5}$ More explicitly, we must consider quark plus antiquark states. However antiquark contributions can be easily restored from the quark calculation. We also consider one flavor with electric charge $e_{q}$.
} 
where $\lambda^{2}$ denotes the artificial photon mass which plays the role of IR scale. Let us note that $\lambda^{2}$ is an IR QCD regulator and is not related with the IR QED regularization. We also do not consider the imaginary parts because all discussed observables are sensitive only to the real part of the TPE amplitudes.

From the discussion in the previous section, it follows that the corresponding amplitude can be written as

$$
\begin{aligned}
A_{e q}^{2 \gamma} & =\frac{4 \pi \alpha}{Q^{2}} \bar{u}_{v} \gamma^{\nu} u_{\bar{v}}\left(\bar{q}_{n} \gamma_{\perp}^{\sigma} q_{\bar{n}}+\bar{v}_{n} \gamma_{\perp}^{\sigma} v_{\bar{n}}\right) \\
& \times\left\{\frac{\alpha}{\pi} g_{1}^{q}(z, Q)+\frac{1}{s} P^{\nu} K_{\perp}^{\sigma} g_{3}^{q}(z, Q)+C_{-}^{\sigma \nu}\left(z, Q^{2}\right) \mathcal{F}_{1}^{q}(Q)\right\},
\end{aligned}
$$

where the FFs $\mathcal{F}_{1}^{q}$ and $g_{1,3}^{q}$ denote the SCET amplitudes computed with quarks. FF $\mathcal{F}_{1}^{q}$ is easily computed from the tree level diagrams and reads

$$
\mathcal{F}_{1}^{q}=e_{q}^{2}+\mathcal{O}\left(\alpha_{s}\right)
$$

The calculation of $g_{1}^{q}$ involves one-loop diagrams shown in Fig.5. These diagrams must be
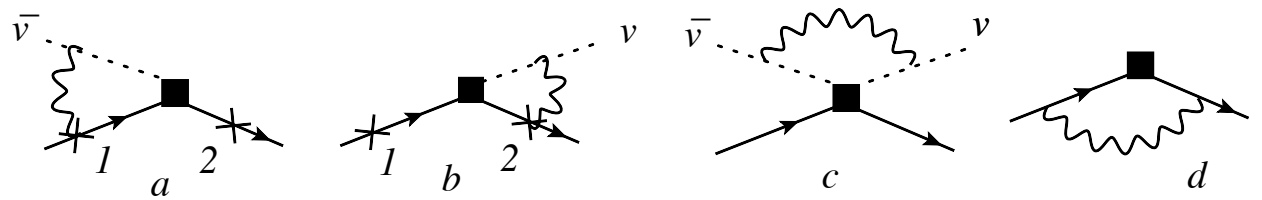

Figure 5. The one-loop diagrams $a, b$ contribute to the SCET perturbative function $g_{1}^{q}$ associated with the TPE contribution. The dashed line with label $v$ and $\bar{v}$ denote the soft WLs, the crossed quark lines show the different attachments of the soft gluon. The diagrams $c$ and $d$ are related to the vertex corrections and therefore were neglected.

computed in SCET-I which describes the coupling of the soft photon with the hard-collinear quarks. The one-loop diagrams in Fig.5 have an UV-divergence which is regularized using dimensional regularization $D=4-2 \varepsilon$. We present the details of this calculation in Appendix C. After UV renormalization the result reads

$$
g_{1}^{q}(z, Q)=\ln \bar{z} \ln \frac{\mu_{F}^{2}}{\lambda^{2}} \mathcal{F}_{1}^{q}, g_{3}^{q}(z, Q)=0 .
$$

Formally, such contribution arises form the mixing between the operators $Y_{v}^{\dagger} S_{v} O_{+}^{\mu}$ and $O_{-}^{\mu}$ due to soft photon interactions.

It is convenient to define the two independent coefficient functions $C_{M, 3}$ as

$$
C_{-}^{\mu \nu}\left(z, Q^{2}\right)=g_{\perp}^{\mu \nu} \frac{\alpha}{\pi} C_{M}\left(z, Q^{2}, \mu_{F}\right)+\frac{1}{s} P^{\nu} K_{\perp}^{\mu} \frac{\alpha}{\pi} C_{3}(z) .
$$

Using the explicit results for $\mathcal{F}_{1}^{q}$ and $g_{1,3}^{q}$ and comparing two expressions for $A_{e q}^{2 \gamma}$ given in Eqs.(4.1) and (4.4) we obtain

$$
g_{\perp}^{\mu \nu} \frac{\alpha}{\pi} C_{M}\left(z, Q^{2}, \mu_{F}\right)+\frac{1}{s} P^{\nu} K_{\perp}^{\mu} \frac{\alpha}{\pi} C_{3}\left(z, Q^{2}\right)=g_{\perp}^{\mu \nu} \frac{\alpha}{\pi}\left(\tilde{f}_{1}-\ln \bar{z} \ln \frac{\mu_{F}^{2}}{\lambda^{2}}\right)+\frac{1}{s} P^{\nu} K_{\perp}^{\mu} \frac{\alpha}{\pi} \tilde{f}_{3},
$$


which yields

$$
\begin{gathered}
C_{M}\left(z, Q^{2}, \mu_{F}\right)=\ln \bar{z} \ln \frac{s}{\mu_{F}^{2}}+\frac{1}{2} \ln ^{2} \bar{z}-\frac{1}{4} \frac{z \ln ^{2} z}{1-z}-\frac{z \ln ^{2} \bar{z} / z}{4}-\frac{1}{2} \ln \bar{z}-\frac{z \pi^{2}}{4}+\frac{\pi^{2}}{2}, \\
C_{3}\left(z, Q^{2}\right)=\frac{2-z}{2 \bar{z}^{2}} \ln ^{2} z-\frac{2-z}{2} \ln ^{2} \frac{\bar{z}}{z}+\frac{1}{\bar{z}} \ln z+\ln \frac{\bar{z}}{z}-\frac{2-z}{2} \pi^{2} .
\end{gathered}
$$

Notice that the soft scale $\lambda^{2}$ cancel in the final expression for $C_{M}$ as it should be. In Eq.(4.9) we write $\ln \left[s / \mu_{F}^{2}\right]$ with the total energy $s$ for convenience.

Therefore our final result for the soft spectator contribution to the TPE amplitude can be written as

$$
\begin{array}{r}
A_{e p}^{2 \gamma}=\frac{4 \pi \alpha}{Q^{2}} \bar{u}_{v} \gamma^{\mu} u_{\bar{v}} \bar{N}\left(p^{\prime}\right) \frac{\not \hbar h}{4} \gamma_{\perp}^{\mu} N(p)\left\{f_{1}(Q)+\frac{\alpha}{\pi} g_{1}\left(z, Q, \mu_{F}\right)+\frac{\alpha}{\pi} C_{M}\left(z, Q^{2}, \mu_{F}\right) \mathcal{F}_{1}(Q)\right\} \\
+\bar{u}_{v} \not P u_{\bar{v}} \bar{N}\left(p^{\prime}\right) \frac{\not h \hbar}{4} \not K_{\perp} N(p) \frac{1}{s} \frac{\alpha}{\pi}\left\{g_{3}(z, Q)+C_{3}\left(z, Q^{2}\right) \mathcal{F}_{1}(Q)\right\} .
\end{array}
$$

Let us now discuss the complete factorization formula describing the TPE contribution. We suggest that the TPE amplitudes in Eq.(2.8) in the region of wide-angle scattering (2.23) can be described by the following tentative factorization formula

$$
\begin{aligned}
\delta \tilde{G}_{M}^{2 \gamma}\left(\varepsilon, Q^{2}\right) & =\delta \tilde{G}_{M}^{(s)}\left(\varepsilon, Q^{2}\right)+\delta \tilde{G}_{M}^{(h)}\left(\varepsilon, Q^{2}\right), \\
\tilde{F}_{3}\left(\varepsilon, Q^{2}\right) & =\tilde{F}_{3}^{(s)}\left(\varepsilon, Q^{2}\right)+\tilde{F}_{3}^{(h)}\left(\varepsilon, Q^{2}\right), \\
\delta \tilde{F}_{2}^{2 \gamma}\left(\varepsilon, Q^{2}\right) & =\delta \tilde{F}_{2}^{(s)}\left(\varepsilon, Q^{2}\right)+\delta \tilde{F}_{2}^{(h)}\left(\varepsilon, Q^{2}\right),
\end{aligned}
$$

where the indices $(s)$ and $(h)$ denote the contributions related to the soft and hard spectator scattering, respectively.

The hard spectator contributions describe the scattering where the all spectator quarks are involved into the hard subprocess. Schematically these contributions are shown by the third diagram on $r h s$ in Fig.1. Schematically one can write

$$
\begin{aligned}
& F_{3}^{(h)}=\mathbf{\Psi}\left(x_{i}\right) * H_{3}\left(z, Q^{2} ; x_{i}, y_{i}\right) * \mathbf{\Psi}\left(y_{i}\right), \\
& \delta \tilde{G}_{M}^{(h)}=\mathbf{\Psi}\left(x_{i}\right) * H_{M}\left(z, Q^{2} ; x_{i}, y_{i}\right) * \boldsymbol{\Psi}\left(y_{i}\right),
\end{aligned}
$$

where symbols $H_{3, M}$ denote the hard coefficient functions and the asterisk is used as notation for the convolution integrals over the collinear fractions $x_{i}, y_{i}$. The leading order coefficient functions $H_{3, M}$ were computed in Refs. [32, 33]. The hard spectator contributions are dominated by one hard gluon exchange, see Fig.2, and are of order $\alpha_{s}$. The nucleon distribution amplitude $\boldsymbol{\Psi}\left(x_{i}\right)$ describes the nonperturbative overlap of the collinear quarks with the nucleon state. The hard spectator contribution to the helicity flip amplitude $F_{2}^{(h)}$ can be written in a similar way but it is power suppressed. Furthermore, its the convolution integrals are ill defined due to the end-point singularities. Therefore we will not consider the helicity flip amplitude in the present publication. 
The soft spectator contributions describe the scattering where the spectator quarks are soft. These TPE contributions have been discussed above. Comparing Eq. (2.8) with Eq. (4.11) (the matching is quite similar to one described in Appendix A) we obtain

$$
\begin{gathered}
\delta \tilde{G}_{M}^{(s)}\left(\varepsilon, Q^{2}\right)=\frac{\alpha}{\pi}\left\{g_{1}\left(z, Q, \mu_{F}\right)+C_{M}\left(z, Q^{2}, \mu_{F}\right) \mathcal{F}_{1}(Q)\right\}, \\
\frac{\nu}{m^{2}} \tilde{F}_{3}^{(s)}=\frac{\alpha}{\pi} \frac{\nu}{s}\left\{g_{3}(z, Q)+C_{3}\left(z, Q^{2}\right) \mathcal{F}_{1}(Q)\right\},
\end{gathered}
$$

where we assume $z \equiv z(\varepsilon)$ and $\nu$ defined in Eq. (2.4).

The soft contribution to the helicity flip amplitude is also suppressed by the power of the large scale $1 / Q^{2}$ and can be written as (for simplicity we do not write the arguments on the $r h s)$

$$
\delta \tilde{F}_{2}^{(s)}\left(\varepsilon, Q^{2}\right)=\left[\delta \tilde{F}_{2}^{(s)}\right]_{\mathrm{subl}}+\frac{\alpha}{\pi} \frac{4 m^{2}}{Q^{2}}\left[g_{1}+C_{M} \mathcal{F}_{1}+\frac{\nu}{s}\left\{C_{3} \mathcal{F}_{1}+g_{3}\right\}\right] .
$$

In this expression we computed only the kinematical corrections similar to the Pauli FF $F_{2}$ in Eq.(3.15). The complete answer includes also contribution with the matrix elements of the subleading SCET operators $\left[\delta \tilde{F}_{2}^{(s)}\right]_{\text {subl }}$ which we do not consider for simplicity.

As we can see from Eqs. $(4.17,4.18)$ the two leading power amplitudes are described by the three nonperturbative functions: the SCET FF $\mathcal{F}_{1}(Q)$ and the SCET amplitudes $g_{1,3}(z, Q)$. If both photons are hard then the soft spectator scattering is described by the pure QCD sector. In this case the hard-collinear dynamics involves only the one hard scale $Q$ and is described by the SCET FF $\mathcal{F}_{1}(Q)$. Therefore the $\varepsilon$-dependence is described by the hard coefficient functions $C_{3, M}$ and can be computed in perturbative QCD. The situation is different when the one photon is soft. Such soft photon interacts with the hard-collinear and soft constituents and therefore the soft-overlap contribution is more complicated and is described by the SCET amplitudes $g_{1,3}\left(z, Q^{2}\right)$. We recall that we assume that the hardcollinear scale is not large and we can not use it for perturbative calculations. In this case the $\varepsilon(z)$-dependence originates from the soft dynamics and can not be computed from pQCD.

Another difficulty is related to the separation of the amplitudes for the soft and hard spectator contributions. The simple sums as in Eqs.(4.12-4.14) are motivated by the structure of the SCET-I operators. However the overlap of the soft and collinear sectors in SCET-II makes such separation ambiguous. As a rule this leads to the end-point singularities in the collinear integrals defining the hard spectator terms. In Ref.[37] it was discussed that such situation even occurs for the FF $F_{1}$ where one could expect that the hard spectator contribution is well defined. In general, the separation between the soft and hard spectators contributions can be well formulated only within a certain regularization scheme which allows to treat the soft and collinear sectors separately and consistently. From this point of view our results are still not complete because we did not provide such a separation scheme. This question will be discussed in the next two sections.

Finally let us note that the QED dynamics considered here is closely connected with the underlying QCD dynamics. In particular the energy and virtuality of the soft photons can 
not be smaller than the typical QCD soft scale $\Lambda$, because otherwise the photon interacts with the total proton charge and can not resolve the constituent charges inside the target. Therefore our IR regulator $\lambda$ (introduced for convenience as photon mass) must cancel in the hard coefficient function which describes the scattering of the hard photons. The standard QED IR-divergence is included in the SCET amplitude $g_{1}$ which is defined via the matrix element in Eq.(3.31).

Let us also note that each separate diagram in Fig.4 also receives contributions associated with the collinear regions (one of the photons is collinear to one of the external momenta). Moreover these collinear regions overlap with the soft one. However in the sum of the diagrams, the collinear contributions cancel and this cancellation is required by gauge invariance because the electric charges of the quarks and leptons are different. A detailed discussion of this point can be found in Appendix D.

In conclusion, we suggested a factorization formula for the TPE soft spectator scattering contribution. We performed the matching and computed the coefficient functions in leading order in approximation $\alpha_{s}$. In order to apply this result in a phenomenological analysis, we next need to define the SCET amplitudes which arise in our approach.

\section{Estimate of the soft spectator contribution with $\mathcal{F}_{1}$ from wide-angle Compton scattering data}

\subsection{QCD factorization for the wide-angle Compton scattering process}

The SCET FF $\mathcal{F}_{1}(Q)$ defined in Eq.(3.24) describes the long distance interactions in the soft spectator contribution with the hard photons. In order to estimate this quantity for different values of $Q^{2}$ one can use the universality of its definition. In other words if one can find another process which is described by the same SCET FF then one can use the corresponding observables in order to estimate $\mathcal{F}_{1}(Q)$. In our case such analysis can be carried out for the wide-angle Compton scattering (WACS). The cross section of this reaction and a polarization asymmetry in the region of large Mandelstam variables (large scattering angles $\theta_{c m} \sim 90^{\circ}$ in the cms frame ) have been measured at JLab [51].

The theoretical description of this reaction has been presented long time ago [53, 54] and it is based on the dominance of the hard spectator scattering which leads to the collinear factorization for the dominant amplitudes. In this case the situation is very similar to the situation with the nucleon electromagnetic FFs. At large energy and momentum transfer $s \sim-t \sim-u \gg \Lambda^{2}$ the leading power contribution to the WACS amplitude $T_{i}$ can be described as a collinear convolution of the hard coefficient function $H_{i}(s, t)$ with the nucleon DAs $\Psi$

$$
T_{i}^{(h)}(s, t)=\mathbf{\Psi}\left(y_{i}\right) * H_{i}\left(s, t ; x_{i}, y_{i}\right) * \mathbf{\Psi}\left(x_{i}\right)
$$

where the asterisk denotes the convolution integrals with respect to the collinear fractions $x_{i}, y_{i}$. The reduced diagram describing the hard spectator scattering is shown in Fig.6. Besides the hard spectator scattering, one can easily find that the soft spectator scattering can also contribute to the same power and that this situation is quite similar to the one 


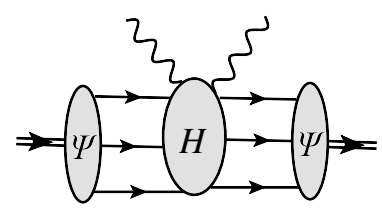

Figure 6. Reduced diagram describing the hard spectator scattering contribution in the WACS.

discussed for the $\mathrm{FF} F_{1}$ in $[31,35]$. For instance, one can investigate the soft region for the diagram in Fig.7 in the same way as it was done for the analogous diagram in case of the FF $F_{1}$ in $[31,35]$. Such analysis allows to see that the contribution from the domain

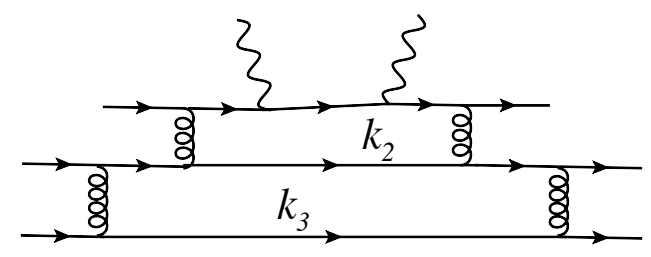

Figure 7. Example of the diagram which has the leading power contribution from the region with the soft spectators $k_{2 \mu} \sim k_{3 \mu} \sim \Lambda$

where momenta $k_{2 \mu} \sim k_{3 \mu} \sim \Lambda$ are soft is suppressed by the same power of $Q^{2}$ as from the domain where these momenta are hard: $k_{2 \mu} \sim k_{3 \mu} \sim Q$. This observation provides the arguments that the complete factorization is described by the soft and hard spectator contributions where the last one is described by the collinear factorization suggested in [53]. Moreover, a phenomenological analysis of the experimental data [51, 52] allows one to conclude that the dominant contribution in the cross section is provided by the soft spectator scattering mechanism. The best description of the existing WACS data has been achieved using the so-called handbag or GPD approach [55-58] which can be considered as a model for the soft spectator scattering. However, the systematic analysis within this approach is rather problematic because the parametrization of the soft overlap contribution by the GPD matrix element is not consistent with the IR-structure of the QCD diagrams, see for instance [57].

In the current section we consider the description of the WACS in the SCET framework. Such consideration is quite similar to the analysis of the nucleon FFs carried out in [31].

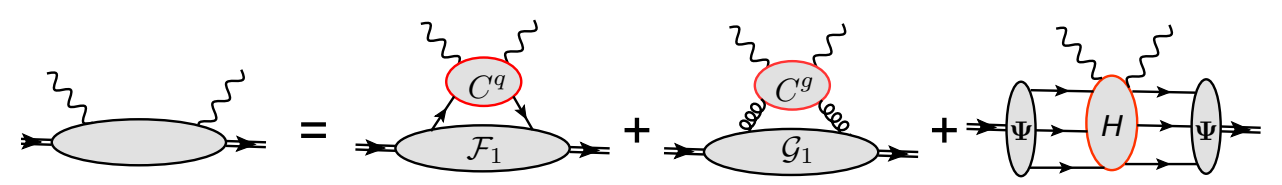

Figure 8. Schematic representation of the WACS factorization. The soft-overlap contributions are described by the SCET FFs $\mathcal{F}_{1}$ and $\mathcal{G}_{1}$.

We expect that in the region $s \sim-t \sim-u \gg \Lambda^{2}$ the WACS amplitudes, denoted by $T_{i}$, can be described within SCET approach by the following tentative factorization formula

$$
T_{i}(s, t)=T_{i}^{(s)}(s, t)+T_{i}^{(h)}(s, t),
$$


where the hard spectator contribution $T^{(h)}$ is given by Eq.(5.1). The soft spectator term reads :

$$
T_{i}^{(s)}(s, t)=C_{i}^{q}(s, t, \mu) \mathcal{F}_{1}(Q, \mu)+C_{i}^{g}(s, t, \mu) \mathcal{G}_{1}(Q, \mu)
$$

where the hard coefficient functions $C_{i}^{q, g}$ describe a hard subprocess, the functions $\mathcal{F}_{1}$ and $\mathcal{G}_{1}$ describe the hard-collinear and soft interactions and can be defined in the framework of the SCET-I approach. We provide their explicit definitions below. In Fig. 8 we illustrate the WACS factorization in terms of reduced diagrams.

In order to proceed further let us introduce the following definitions. The amplitude of the process $\gamma(k)+p(p) \rightarrow \gamma\left(k^{\prime}\right)+p\left(p^{\prime}\right)$ can be written as [59]

$$
\left.\left\langle p^{\prime}, k^{\prime} \text { out }\right| \text { in } k, p\right\rangle=i(2 \pi)^{4} \delta\left(p+k+p^{\prime}+k^{\prime}\right) \varepsilon^{* \mu}\left(k^{\prime}\right) \varepsilon^{\nu}(k) e^{2} T^{\mu \nu},
$$

with

$$
T^{\mu \nu}=i \int d^{4} x e^{-i(k x)}\left\langle p^{\prime}\left|T\left\{J_{e m}^{\mu}(0) J_{e m}^{\nu}(x)\right\}\right| p\right\rangle
$$

where $J_{e m}^{\mu}$ represents the quark electromagnetic current

$$
J_{e m}^{\mu}(x)=\sum_{q} e_{q} \bar{q}(x) \gamma^{\mu} q(x)
$$

The hadronic matrix element $T^{\mu \nu}$ is parametrized in terms of six independent scalar amplitudes $T_{i}$ as

$$
\begin{aligned}
T^{\mu \nu}= & \bar{N}\left(p^{\prime}\right)\left\{-\frac{P^{\prime \mu} P^{\prime \nu}}{P^{\prime 2}}\left(T_{1}+\not K T_{2}\right)-\frac{N^{\mu} N^{\nu}}{N^{2}}\left(T_{3}+\not K T_{4}\right)\right. \\
& \left.+\frac{P^{\prime \mu} N^{\nu}-P^{\prime \nu} N^{\mu}}{P^{\prime 2} K^{2}} i \gamma_{5} T_{5}+\frac{P^{\prime \mu} N^{\nu}+P^{\prime \nu} N^{\mu}}{P^{\prime 2} K^{2}} i \gamma_{5} \not K T_{6}\right\} N(p) .
\end{aligned}
$$

In the last formula we used

$$
\begin{gathered}
P=\frac{1}{2}\left(p+p^{\prime}\right), P^{\prime}=P-K \frac{(P . K)}{K^{2}}, K=\frac{1}{2}\left(k+k^{\prime}\right), \\
N^{\mu}=\varepsilon[\mu \alpha \beta \gamma] P^{\alpha} \frac{1}{2}\left(p-p^{\prime}\right)^{\beta} K^{\gamma}, \quad \varepsilon_{0123}=+1, \\
s=(p+k)^{2}, t=\left(p^{\prime}-p\right)^{2}=-Q^{2}, z=\frac{-t}{s} \simeq \frac{1}{2}\left(1-\cos \theta_{c m}\right) .
\end{gathered}
$$

One can see from Eq.(5.7) that three amplitudes $T_{2,4,6}$ describe the Compton scattering without nucleon helicity flip, while the three others describe the amplitudes with helicity flip.

We again consider a Breit-like frame and therefore the light-cone expansions of the momenta can be easily obtained from Eq. (3.2) substituting the lepton momenta $k$ and $k^{\prime}$ by photon momenta $q$ and $q^{\prime}$, respectively. Therefore the formulation of the appropriate SCET degrees of freedom in the collinear sectors associated with $n$ and $\bar{n}$ light-cone directions is the same as discussed in Section 3. 
In the following we only discuss the factorization for the soft spectator contribution. The SCET-I factorization implies that the $T$-product of the electromagnetic currents in Eq.(5.5) can be represented as the convolution of the coefficient function with a certain SCET-I operator constructed from the SCET-I fields $\xi_{n}, A_{\mu}^{(n)}, \xi_{\bar{n}}, A_{\mu}^{(\bar{n})}$. This schematically can be written as

$$
T\left\{J_{e m}^{\mu}(0) J_{e m}^{\nu}(x)\right\}=\sum_{i} \tilde{C}_{i}^{\mu \nu} * O_{i}\left[\xi_{n}, A^{(n)}, \xi_{\bar{n}}, A^{(\bar{n})}\right],
$$

where the asterisk denotes the convolution integrals in the coordinate space. We assume that the dominant contribution in the wide-angle kinematics is provided by appropriate operators with the minimal dimension according to SCET-I counting rules, see Refs. [4045]. Furthermore in our analysis of the soft spectator scattering contribution we consider as leading power terms only the contributions associated with the SCET-I counting, i.e. we consider the powers of the large scale $Q$ arising from factorization of the hard modes. We do not consider the full power counting analysis which includes also the SCET-II counting. We consider the region where the hard-collinear scale is still small, and therefore we can not perform an expansion with respect to this scale.

In our case we find that the set of the suitable operators which is consistent with the symmetries and has the minimal SCET-I dimension consists of the quark operator $O_{-}^{\alpha}\left(\lambda_{1}, \lambda_{2}\right)$ defined in Eq.(3.17) and of the pure gluon operator $O_{g}^{\alpha \beta}\left(\lambda_{1}, \lambda_{2}\right)$ which includes the transverse gluon fields $A_{\mu \perp}^{(n)}, A_{\mu \perp}^{(\bar{n})}$ and can be written as

$$
O_{g}^{\alpha \beta}\left(\lambda_{1}, \lambda_{2}\right)=\operatorname{Tr}\left\{\left[W_{n}^{\dagger}\left(\lambda_{1} \bar{n}\right) D_{\alpha \perp} W_{n}\left(\lambda_{1} \bar{n}\right)\right]\left[W_{\bar{n}}^{\dagger}\left(\lambda_{2} n\right) D_{\beta \perp} W_{\bar{n}}\left(\lambda_{2} n\right)\right]\right\},
$$

where the covariant derivative $i D_{\mu}=i \partial_{\mu}+g A_{\mu}^{(n, \bar{n})}$ acts on the fields only inside the square brackets [...]. Below we restrict our discussion only to the leading order in $\alpha_{s}$. Then we can skip the discussion of the gluon operator because the corresponding hard coefficient function arises only at the next-to-leading order. Therefore at leading order, we only have a contribution only due to the quark operator $O_{-}^{\alpha}\left(\lambda_{1}, \lambda_{2}\right)$ that yields

$$
T\left\{J_{e m}^{\mu}(0) J_{e m}^{\nu}(x)\right\} \simeq \tilde{C}_{h}^{\mu \nu \alpha} * O_{-}^{\alpha}\left(\lambda_{1}, \lambda_{2}\right) .
$$

Substituting this into the matrix element in Eq.(5.5) and using Eq.(3.24) we obtain

$$
T^{\mu \nu} \simeq C_{h}^{\mu \nu \alpha}(s, t) \bar{N}\left(p^{\prime}\right) \frac{\not h \hbar}{4} \gamma_{\perp}^{\alpha} N(p) \mathcal{F}_{1}(Q),
$$

where $C_{h}^{\mu \nu \alpha}(s, t)$ corresponds to the coefficient function in momentum space. From Eq.(5.12) we can conclude that all six amplitudes $T_{i}$ introduced in Eq.(5.5) are described in terms of one SCET FF $\mathcal{F}_{1}$. However helicity flip structures in this case can be obtained only as subleading contributions. We also observe that the dependence on the total energy $s$ is completely described by the hard coefficient function.

In order to compute $C_{h}^{\mu \nu \alpha}$ one has to perform the matching for the tree level diagrams shown in Fig.9. We skip the details of this relatively simple calculation and provide the 


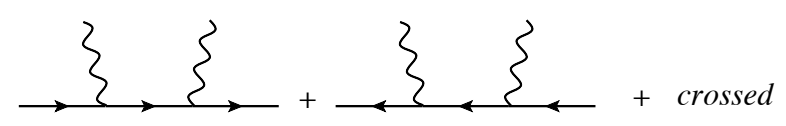

Figure 9. Leading order diagrams to the hard coefficient function describing the soft spectator contribution in WACS.

results for the WACS amplitudes defined in Eq.(5.7). For the invariant amplitudes $T_{i}^{(s)}$ we obtain $(Q=\sqrt{-t}, m$ is nucleon mass $)$

$$
\begin{gathered}
T_{2}^{(s)}=-T_{4}^{(s)}=-\frac{2 s+t}{s(s+t)} \mathcal{F}_{1}(Q), \quad T_{6}^{(s)}=-\frac{t}{s(s+t)} \mathcal{F}_{1}(Q), \\
T_{1}^{(s)}=\frac{m}{-t} \frac{1}{2} \frac{(2 s+t)^{2}}{s(s+t)} \mathcal{F}_{1}(Q)+\bar{T}_{1}^{(s)}, T_{3}^{(s)}=\frac{m}{t} \frac{1}{2} \frac{(2 s+t)^{2}}{s(s+t)} \mathcal{F}_{1}(Q)+\bar{T}_{3}^{(s)}, T_{5}^{(s)}=\bar{T}_{5}^{(s)} .
\end{gathered}
$$

where the terms $\bar{T}_{i}^{(s)}$ appearing on rhs of Eqs.(5.14) denote contributions associated with the subleading SCET operators which have not been considered in our analysis. From these equations one can conclude that helicity flip amplitudes $T_{1,3,5}^{(s)}$ are suppressed by a power of $1 / Q$ (to see this explicitly one has to pass to dimensionless amplitudes in Eq.(5.7)).

The separation of the hard and soft contributions as described in Eq.(5.2) is not simple because it usually implies an additional regularization. Such regularization is required in order to separate the soft and collinear sectors and to describe correctly the so-called large rapidity logarithms, see for instance Refs.[60-63] and references therein. Such problem is clearly seen when one computes the hard spectator contributions, resulting in collinear convolution integrals which are divergent in the end-point region. This is a clear indication that one has to consider the overlap of the soft and hard spectator contributions.

In Ref.[37] the soft and hard spectator contributions describing the FF $F_{1}$ has been investigated and it was demonstrated that in this case the problem of the soft-collinear overlap also exists. We suppose that collinear factorization describing the hard spectator contribution in the TPE and WACS is also violated due to specific end-point divergencies. Therefore we suppose that the most optimal way to proceed further is to explore the socalled physical subtraction scheme suggested in $[64,65]$ and used in the description of different $B$-decay processes.

The idea of this approach is to exclude the $\mathrm{FF} \mathcal{F}_{1}$ from the expression for the physical amplitudes and write the relations between the physical amplitudes directly. In our case, using Eqs.(5.3) (neglecting the gluon contribution at leading order) and Eqs.(5.1) we can write

$$
\mathcal{F}_{1}(Q)=\frac{T_{2}(s, t)}{C_{2}(s, t)}-\mathbf{\Psi}\left(y_{i}\right) * \frac{H_{2}\left(s, t ; x_{i}, y_{i}\right)}{C_{2}(s, t)} * \mathbf{\Psi}\left(x_{i}\right) .
$$

Such formal expression implies that we use some regularization in order to define the divergent quantities in both sides of (5.15). Substituting expression (5.15) into Eq. (5.3) 
for $T_{4,6}$ we obtain

$$
\begin{aligned}
T_{i}(s, t) & =C_{i}(s, t) \frac{T_{2}(s, t)}{C_{2}(s, t)} \\
& +\boldsymbol{\Psi}\left(y_{i}\right) *\left\{H_{i}\left(s, t ; x_{i}, y_{i}\right)-\frac{H_{2}\left(s, t ; x_{i}, y_{i}\right)}{C_{2}(s, t)}\right\} * \boldsymbol{\Psi}\left(x_{i}\right), i=4,6 .
\end{aligned}
$$

The end-point singularities arising in the collinear convolution integrals $\boldsymbol{\Psi} * H_{i} * \boldsymbol{\Psi}$ must cancel in the expression in Eq.(5.16) because the other terms with the physical amplitudes do not have any problems. Hence we can study the relations between the physical amplitudes $T_{i}$ systematically order by order in $\alpha_{s}$. Taking into account that the coefficient functions $H_{i}$ are of order $\alpha_{s}^{2}$ and therefore assuming that the corresponding corrections in Eq. (5.16) are small we obtain the leading order relations

$$
\begin{aligned}
& T_{4}(s, t)=C_{4}(s, t) \frac{T_{2}(s, t)}{C_{2}(s, t)}=-T_{2}(s, t)+\mathcal{O}\left(\alpha_{s}\right), \\
& T_{6}(s, t)=C_{6}(s, t) \frac{T_{2}(s, t)}{C_{2}(s, t)}=\frac{t}{2 s+t} T_{2}(s, t)+\mathcal{O}\left(\alpha_{s}\right) .
\end{aligned}
$$

where the coefficient functions $C_{2,4,6}$ have been computed above to the leading order accuracy. Let us also note that the next-to-leading corrections to these relations arise from the corrections to the coefficient functions $C_{2,4,6}$.

The nice feature is that the SCET FF $\mathcal{F}_{1}$ does not depend on $s$. Therefore this allows one to substitute Eq.(5.15) into other expressions involving the $\mathrm{FF} \mathcal{F}_{1}$, allowing to make predictions for different values of $s$. This is important for our case because the cross sections for the elastic $e p$-scattering have been measured for different values of $s$.

From Eqs. $(5.17,5.18)$ one can easily find that to leading order accuracy the ratios $T_{i} / C_{i}$ satisfy

$$
\frac{T_{2}(s, t)}{C_{2}(s, t)} \simeq \frac{T_{4}(s, t)}{C_{4}(s, t)} \simeq \frac{T_{6}(s, t)}{C_{6}(s, t)} .
$$

Therefore it is convenient to introduce the following notation

$$
\mathcal{R}(s, t)=\frac{T_{2}(s, t)}{C_{2}(s, t)}=-\frac{s(s+t)}{2 s+t} T_{2}(s, t)+\mathcal{O}\left(\alpha_{s}\right) .
$$

Contrary to the SCET FF $\mathcal{F}_{1}$ the ratio $\mathcal{R}$ is a well defined quantity free from the rapidity regularization dependence. ${ }^{6}$ This ratio formally depends on the two variables $s$ and $t$. However, in the region where the QCD factorization is applicable and where power and higher order in $\alpha_{s}$ corrections are small the dependence on $s$ must be small, i.e. we must observe that

$$
\frac{d \mathcal{R}(s, t)}{d s} \simeq 0
$$

which provides a good criterium of the applicability of our approximations. The ratio $\mathcal{R}$ for the different values of $s$ can be extracted from the cross section for WACS measured at JLab [51]. Then this ratio can be used for the calculation of the TPE amplitude defined in Eqs. $(4.17,4.18)$.

\footnotetext{
${ }^{6}$ We assume that the coefficient function $C_{2}$ in Eq.(5.20) is computed at factorization scale $\mu^{2}=-t$.
} 


\subsection{Phenomenological analysis of the WACS observables and extraction of the ratio $\mathcal{R}$}

The unpolarized cross section describing Compton scattering reads [59]

$$
\frac{d \sigma}{d t}=\frac{\pi \alpha^{2}}{\left(s-m^{2}\right)^{2}} W_{00},
$$

with

$$
\begin{aligned}
W_{00} & =\frac{1}{2}\left(s-m^{2}\right)\left(m^{2}-u\right)\left(\left|T_{2}\right|^{2}+\left|T_{4}\right|^{2}\right)+\left(m^{4}-s u\right)\left|T_{6}\right|^{2} \\
& +m(s-u) \operatorname{Re}\left[T_{1} T_{2}^{*}+T_{3} T_{4}^{*}\right]+\frac{1}{2}\left(4 m^{2}-t\right)\left(\left|T_{1}\right|^{2}+\left|T_{3}\right|^{2}\right)-t\left|T_{5}\right|^{2} .
\end{aligned}
$$

Substituting the expressions for $T_{i}$ into Eq. (5.23) and neglecting the subleading power corrections we obtain

$$
\frac{d \sigma}{d t} \simeq \frac{2 \pi \alpha^{2}}{\left(s-m^{2}\right)^{2}}\left(\frac{1}{1-z}+1-z\right)|\mathcal{R}|^{2}=\frac{d \sigma^{\mathrm{KN}}}{d t}|\mathcal{R}|^{2},
$$

where $d \sigma^{\mathrm{KN}}$ is the Klein-Nishina cross section corresponding with a point-like massless particle. This formula is very similar to the one obtained in the handbag approach [55] except for the definition of the form factor.

There are two more observables which describe the correlations of the recoil polarization with the polarization of the photons

$$
K_{L L}=\frac{\sigma_{\|}^{R}-\sigma_{\|}^{L}}{\sigma_{\|}^{R}+\sigma_{\|}^{L}}, \quad K_{L S}=\frac{\sigma_{\perp}^{R}-\sigma_{\perp}^{L}}{\sigma_{\perp}^{R}+\sigma_{\perp}^{L}},
$$

where $\|(\perp)$ refers to a longitudinally (transversely) polarized nucleon and $R(L)$ denotes a right (left) handed photon. For the first one we obtain (again neglecting the power corrections)

$$
K_{L L} \simeq K_{L L}^{\mathrm{KN}}=\frac{2 z(2-z)}{(2-z)^{2}+z^{2}} .
$$

In this case the SCET FF cancels out and we simply reproduce the Klein-Nishina result for the point-like massless particle. The second asymmetry $K_{L S}$ also depends on the nucleon helicity flip amplitudes $T_{1,3,5}$. In order to estimate $K_{L S}$ we use for these amplitudes the expressions obtained in Eqs. (5.14) where we neglect the contributions associated with the subleading SCET operators $\bar{T}_{i}^{(s)}=0$. Then the leading order expression reads

$$
K_{L S}^{\mathrm{LO}} \simeq-\frac{m}{\sqrt{s}} \sqrt{\frac{z}{1-z}} \frac{(2-z)(3 z-2)}{(2-z)^{2}+z^{2}}
$$

where $m$ denotes the nucleon mass. These asymmetries have been measured in JLab [52] at $s=6.9 \mathrm{GeV}^{2}$ and $t=-4.0 \mathrm{GeV}^{2}$

$$
K_{L L}^{\exp }=0.678 \pm 0.083, \quad K_{L S}^{\exp }=0.114 \pm 0.078 .
$$


Using our approximate formulas (5.26) and (5.27) we obtain for these kinematics

$$
K_{L L}^{\mathrm{KN}}=0.70, \quad K_{L S}^{\mathrm{LO}}=0.066 .
$$

The expression for the longitudinal polarization is in good agreement while the transverse polarization is about a factor two smaller but still within the error bars. Notice however, that in these kinematics the value of the $u \simeq-1.14 \mathrm{GeV}^{2}$ is not large compared to the nucleon mass, so that potentially one can expect relatively large power corrections. Therefore the good agreement with experiment is probably due to a compensation of such corrections in higher orders. Nevertheless this analysis of the recoil polarizations allows us to make the following qualitative conclusion: it seems that the symmetry relations (5.17) and (5.18) provides a reasonable approximation for WACS. Therefore it allows us to use the data for the unpolarized cross section and formula (5.24) in order to extract information about the function $\mathcal{R}$.

In order to extract the values $|\mathcal{R}|$ from the data we take the data for the WACS from [51] for which $|t| \geq 2.5 \mathrm{GeV}^{2}$ and $|u| \geq 2.5 \mathrm{GeV}^{2}$. Then for these points we extract the quantity

$$
\left|\mathcal{R}^{\exp }\right|=\sqrt{\frac{d \sigma^{\exp }(s, t)}{d \sigma^{\mathrm{KN}}(s, t)}} .
$$

In Fig.11 we show the results for the $\left|\mathcal{R}^{\exp }\right|$ extracted from the data at three different values of energy: $s=6.8,8.9$ and $10.9 \mathrm{GeV}^{2}$ and $2.5<-t<6.5 \mathrm{GeV}^{2}$. As one can see from this plot, the extracted values $\left|\mathcal{R}^{\exp }\right|$ do not show any dependence on the values of $s$ as required by factorization. Therefore we fit the $Q^{2}$ dependence of the data by the simple empirical function

$$
\mathcal{R}(Q)=\frac{c}{\ln ^{2} Q^{2} / \Lambda^{2}},
$$

which gives $c=0.10 \pm 0.01$ and $\Lambda=1.08 \pm 0.03 \mathrm{GeV}$.

In order to see the sensitivity of our approach on the possible power corrections we also perform the same extractions keeping the exact kinematics in the formula for the cross section. In this case we see that the obtained values for $\left|\mathcal{R}^{\exp }\right|$ are larger, especially in the region of smaller $-t$ and the extracted values also show higher sensitivity on $s$. The solid line in this panel shows the same fit (5.31) which in this case yields $c=0.13 \pm 0.01$ and $\Lambda=1.09 \pm 0.02 \mathrm{GeV}$. From this analysis we can conclude that the data at smaller values of $|u|$ or $|t|$ potentially may obtain sizable corrections from the power suppressed contributions. However because our calculation is incomplete this estimate can only be considered as a qualitative approximation. In our numerical calculation of TPE amplitudes we will use the results obtained without subleading power corrections.

\section{Estimate of the amplitudes $g_{1,3}$ in a hadronic model}

Unfortunately the extraction of the amplitudes $g_{1,3}$ from another measurable process is at present not feasible. Therefore one has to build a model in order to estimate these quantities. 


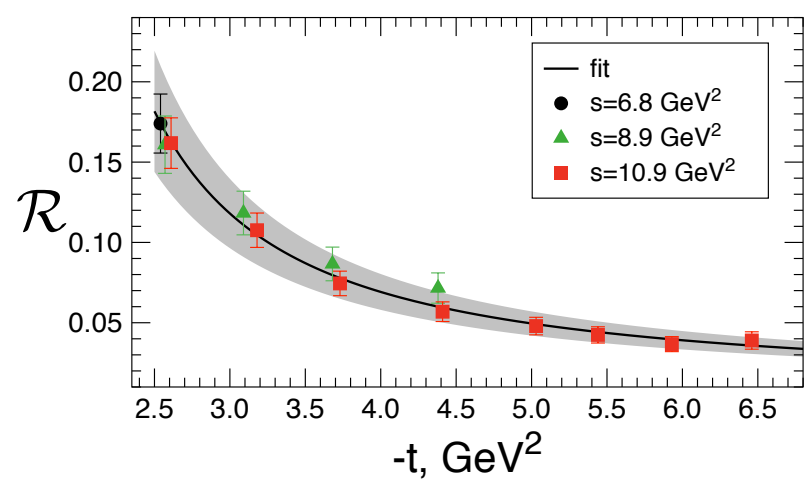

Figure 10. The ratio $\mathcal{R}$ extracted from the WACS data [51] and the fit (5.31) without kinematic power corrections. The gray band shows the $1 \sigma$ error bands.

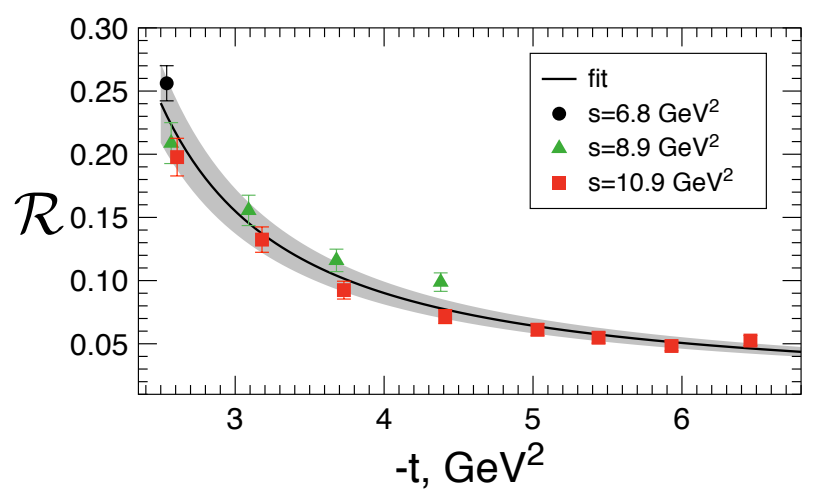

Figure 11. The same as in Fig.10 but including the kinematic power corrections.

As we discussed above, the splitting of the physical amplitudes into soft and hard spectator contributions implies a certain rapidity regularization which allows one to consider the collinear and soft sectors independently. In the present paper we do not consider such scenario for $g_{1,3}$ and perform the numerical estimates of the hard spectator contributions defined in Eqs. $(4.16,4.15)$ using the nucleon distribution amplitude which does not provide the end-point singularities in the collinear convolution integrals. This allows us to consider the SCET matrix element which defines the amplitudes $g_{1,3}$ in (3.31) as a well defined quantity.

The SCET amplitude $g_{1}$ depends on the factorization scale $\mu_{F}$. This factorization scale arises from the factorization of the QED box graphs where $\mu_{F}$ separates the hard photon configuration from the regions with soft virtualities. The evolution equation with respect to this scale can be obtained from the QED renormalization of the SCET operator $Y_{v}^{\dagger} S_{v} O_{+}^{\mu}$, see Eq.(4.6). Equivalently it can be obtained from the requirement that the physical amplitude does not depend on $\mu_{F}$. Using Eq.(4.11) yields

$$
\mu_{F} \frac{d}{d \mu_{F}} A_{e p}^{2 \gamma}=0 \Leftrightarrow \mu_{F} \frac{d}{d \mu_{F}} g_{1}\left(z, Q, \mu_{F}\right)+\mu_{F} \frac{d}{d \mu_{F}} C_{M}\left(z, Q^{2}, \mu_{F}\right) \mathcal{F}_{1}=0
$$


From the expression for $C_{M}$ in Eq.(4.9) we obtain

$$
\mu_{F} \frac{d}{d \mu_{F}} g_{1}\left(z, Q, \mu_{F}\right)=2 \ln \bar{z} \mathcal{F}_{1}(Q) .
$$

The solution of this evolution equation reads

$$
g_{1}\left(z, Q, \mu_{F}\right)=g_{1}\left(z, Q, \mu_{0}\right)+\ln \bar{z} \ln \frac{\mu_{F}^{2}}{\mu_{0}^{2}} \mathcal{F}_{1}(Q),
$$

where $g_{1}\left(z, Q, \mu_{0}\right)$ denotes some initial condition. The logarithmic contribution in Eq.(6.3) can be absorbed into the coefficient function $C_{M}$. In order to estimate the contribution associated with the amplitude $g_{1}$ we have to fix $\mu_{F}=\mu_{0}$ and evaluate $g_{1}\left(z, Q, \mu_{0}\right)$.

Let us firstly consider an appropriate choice of the scale $\mu_{0}$. As we have mentioned before, in factorizing the hard modes we pass to the SCET-I which describes the hardcollinear dynamics. Therefore one can expect that it is natural to assume that $\mu_{0} \sim \sqrt{\Lambda Q}$. However the contributions associated with the hard-collinear photons cancel in the sum of the TPE diagrams. This allows us to assume that the factorization scale $\mu_{F}$ separates the hard and soft electromagnetic configurations in the box diagrams. Using this interpretation we can fix the scale $\mu_{0}$ to be of order $\Lambda$. With such choice $g_{1}\left(\mu_{0} \simeq \Lambda\right)$ describes the TPE configurations where the soft photon carries momentum $q_{i \mu} \lesssim \Lambda$. Notice that in this case the virtuality of the involved active quark remains hard-collinear as it should be. Such choice for $\mu_{F}$ explains its decoupling from the QCD factorization scale $\mu \sim \sqrt{\Lambda Q}$ which enters in the definition of the QCD SCET FFs. Let us also mention that choosing $\mu_{0} \simeq \Lambda$ one obtains the large logarithm in the coefficient function: $C_{M}\left(\mu_{F}=\mu_{0}\right) \sim \mathcal{F}_{1} \ln \bar{z} \ln \frac{s}{\mu_{0}^{2}}$. However the combination $\frac{\alpha}{\pi} \ln \frac{s}{\mu_{0}^{2}}$ is still quite small for the practically relevant values of $s$ therefore we do not need to sum such contributions.

Taking into account that the amplitude $g_{1}$ describes the highly asymmetrical configuration where one of the photons carries the momentum $q_{i \mu} \lesssim \Lambda$ one can conclude that it must also include the IR-sensitive QED contribution. Such term is very important for the correct treatment of the QED IR-divergencies. In what follows we suppose that the soft photon virtualities which are relevant for the QED IR-divergent part are much smaller than the QCD soft virtualities of order $\Lambda: q_{i}^{2} \ll \Lambda^{2}$. Such ultrasoft photons can interact only with the point-like proton and their dynamics can be described in the framework of some effective theory with the nucleon degrees of freedom. These arguments suggest that we can try to estimate $g_{1}\left(\mu_{0} \sim \Lambda\right)$ using a model with a nucleon and excited resonance states. We may expect that such model can provide a reasonable estimate if the dominant contribution to $g_{1,3}\left(\mu_{0} \sim \Lambda\right)$ arises from the region of ultrasoft photon. From this point of view such calculation is quite similar to hadronic model calculations performed before, with the one important difference: the virtuality of the soft photon is constrained by $\mu_{0}^{2} \sim \Lambda^{2}$ and the hard dynamics is factorized into the hard coefficient function $C_{+}$.

In order to perform our calculation we proceed as follows. First, we insert the inter- 
mediate nucleon states approximating the matrix element

$$
\begin{aligned}
& \left\langle p^{\prime}\left|T\left\{O_{+}^{\mu},\left(Y_{v}^{\dagger} S_{\bar{v}}-1\right)\right\}\right| p\right\rangle_{\mathrm{SCET}} \simeq \\
& \quad \int \frac{d^{D} r}{(2 \pi)^{D}} \sum_{R, s}\left\langle p^{\prime}\left|O_{+}^{\mu}\right| r, s\right\rangle_{\mathrm{SCET}} \frac{i}{r^{2}-m_{R}^{2}}\left\langle r, s\left|\left(Y_{v}^{\dagger} S_{\bar{v}}-1\right)\right| p\right\rangle \\
& \quad+\int \frac{d^{D} r}{(2 \pi)^{D}} \sum_{R, s}\left\langle p^{\prime}\left|\left(Y_{v}^{\dagger} S_{\bar{v}}-1\right)\right| r, s\right\rangle \frac{i}{r^{2}-m_{R}^{2}}\left\langle r, s\left|O_{+}^{\mu}\right| p\right\rangle_{\mathrm{SCET}},
\end{aligned}
$$

where $R$ denotes the nucleon resonance state and $s$ describes its polarization. In Eq.(6.4) we wrote the combination $Y_{v}^{\dagger} S_{\bar{v}}-1$ in order to stress that we consider only the next-to-leading QED contributions. Let us again mention that we consider only the TPE interactions of the soft photons with hadrons neglecting the electron and hadron vertex contributions. The matrix elements with the soft photon WLs can be computed in the effective theory with the nucleon degrees of freedom.

$$
\left\langle r, s\left|\left(Y_{v}^{\dagger} S_{\bar{v}}-1\right)\right| p\right\rangle \simeq \int d x\left\langle r, s\left|T\left\{\left(Y_{v}^{\dagger} S_{\bar{v}}-1\right), J_{R}^{\alpha}(x) i e B_{\alpha}^{(s)}(x)\right\}\right| p\right\rangle,
$$

where $J_{R}^{\alpha}(x)$ denotes the electromagnetic current of the hadron $R$. Contracting the photon fields we obtain

$$
\begin{aligned}
& T\left\{\left(Y_{v}^{\dagger} S_{\bar{v}}-1\right), i e B_{\alpha}^{(s)}(x)\right\} \simeq \\
& T\left\{-i e \int_{-\infty}^{0} d t \bar{v} \cdot B^{(s)}(t \bar{v})-i e \int_{0}^{\infty} d t v \cdot B^{(s)}(t v), i e B_{\alpha}^{(s)}(x)\right\} \\
& \quad=e^{2} \int \frac{d^{D} l}{(2 \pi)^{D}} e^{-i(l x)} \frac{(-i)}{\left[l^{2}+i \varepsilon\right]}\left\{\frac{i \bar{v}^{\alpha}}{[-(\bar{v} l)+i \varepsilon]}+\frac{i v^{\alpha}}{[(v l)+i \varepsilon]}\right\} .
\end{aligned}
$$

Substitution (6.7) into (6.5) gives

$$
\begin{aligned}
& \left\langle r, s\left|\left(Y_{v}^{\dagger} S_{\bar{v}}-1\right)\right| p\right\rangle \simeq \\
& \quad e^{2}\left\langle r, s\left|J_{R}^{\alpha}(0)\right| p\right\rangle \int \frac{d^{D} l}{\left[l^{2}+i \varepsilon\right]} \delta^{D}(r-l+p)\left\{\frac{\bar{v}^{\alpha}}{[-(\bar{v} l)+i \varepsilon]}+\frac{v^{\alpha}}{[(v l)+i \varepsilon]}\right\} .
\end{aligned}
$$

An analogous expression can also be obtained for the second matrix element in Eq.(6.4). Substituting these expressions into Eq.(6.4) and integrating over $d^{D} r$ yields

$$
\begin{aligned}
\left\langle p^{\prime}\left|T\left\{O_{+}^{\mu},\left(Y_{v}^{\dagger} S_{\bar{v}}-1\right)\right\}\right| p\right\rangle_{\mathrm{SCET}} \simeq \frac{e^{2} \mu_{\mathrm{uv}}^{2 \varepsilon}}{(2 \pi)^{D}} \int \frac{d^{D} l}{\left[l^{2}+i \varepsilon\right]} & \left\{\frac{\bar{v}^{\alpha}}{[-(\bar{v} l)+i \varepsilon]}+\frac{v^{\alpha}}{[(v l)+i \varepsilon]}\right\} \\
& \times \sum_{R} M_{R}^{\mu \alpha}(l),
\end{aligned}
$$

where

$$
\begin{aligned}
M_{R}^{\mu \alpha}(l) & =\sum_{s}\left\langle p^{\prime}\left|O_{+}^{\mu}\right| p+l, s\right\rangle_{\mathrm{SCET}} \frac{i}{(p+l)^{2}-m_{R}^{2}}\left\langle p+l, s\left|J_{R}^{\alpha}(0)\right| p\right\rangle \\
& +\sum_{s}\left\langle p^{\prime}\left|J_{R}^{\alpha}(0)\right| p^{\prime}-l, s\right\rangle \frac{i}{\left(p^{\prime}-l\right)^{2}-m_{R}^{2}}\left\langle p^{\prime}-l, s\left|O_{+}^{\mu}\right| p\right\rangle_{\mathrm{SCET}} .
\end{aligned}
$$


This representation can be easily understood as a calculation of the diagrams in Fig.12. We use dimensional regularization with $D=4-2 \varepsilon$ in order to regularize the UV-divergencies, see further discussion.

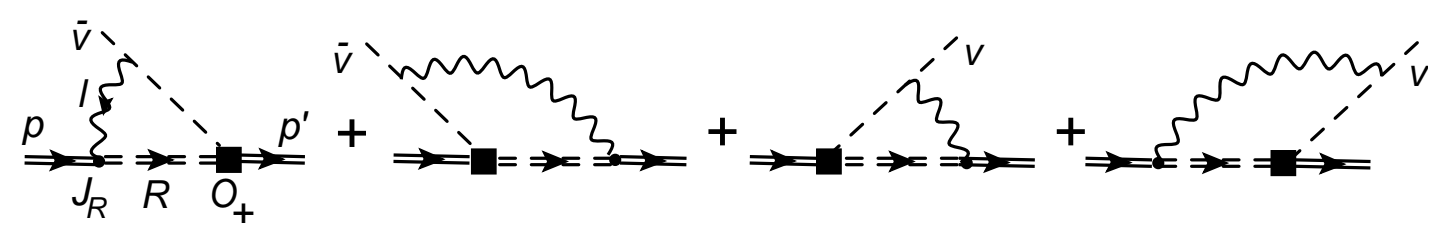

Figure 12. The diagrams in the low-energy effective theory describing the expression for the amplitude proportional to $g_{1}$. The simple dashed line denotes WLs associated with vectors $v$ and $\bar{v}$, the black square denotes the vertex with $O_{\mu}^{+}$.

In order to describe correctly the region associated with the small momentum $l$ (soft photon) we need to perform in Eq.(6.10) some approximations. Namely, we neglect the small momentum $l$ in the numerators and denominators in Eq.(6.10).

$$
\begin{aligned}
& \left\langle p^{\prime}\left|O_{+}^{\mu}\right| p+l, s\right\rangle \simeq\left\langle p^{\prime}\left|O_{+}^{\mu}\right| p, s\right\rangle_{\mathrm{SCET}}, \quad\left\langle p+l, s\left|J_{R}^{\alpha}(0)\right| p\right\rangle \simeq\left\langle p, s\left|J_{R}^{\alpha}(0)\right| p\right\rangle . \\
& \frac{i}{(p+l)^{2}-m_{R}^{2}} \simeq \frac{i}{p_{+} l_{-}+m^{2}-m_{R}^{2}}, \quad \frac{i}{\left(p^{\prime}-l\right)^{2}-m_{R}^{2}} \simeq \frac{i}{-p_{-}^{\prime} l_{+}+m^{2}-m_{R}^{2}} .
\end{aligned}
$$

In these formulas we do not skip the masses because

$$
p_{+} l_{-} \lesssim \Lambda Q, \quad m^{2}-m_{R}^{2} \simeq\left(m-m_{R}\right)\left(m+m_{R}\right) \sim \Lambda\left(m+m_{R}\right),
$$

and therefore the difference $m^{2}-m_{R}^{2}$ is potentially comparable with the hard-collinear scale in the region of moderate values of $Q$ where $\Lambda Q \sim m^{2}$. Moreover this difference of the masses provides also a natural regulator for the QED IR-divergence. In this case only the elastic contribution has an IR-divergence. Therefore we can write

$$
\begin{aligned}
\left\langle p^{\prime}\left|O_{+}^{\mu}\left(Y_{v}^{\dagger} S_{\bar{v}}-1\right)\right| p\right\rangle_{\mathrm{SCET}}=\frac{e^{2} \mu_{\mathrm{uv}}^{2 \varepsilon}}{(2 \pi)^{D}} & \int \frac{d^{D} l}{\left[l^{2}-\lambda^{2}+i \varepsilon\right]}\left\{\frac{\bar{v}^{\alpha}}{[-(\bar{v} l)+i \varepsilon]}+\frac{v^{\alpha}}{[(v l)+i \varepsilon]}\right\} \\
& \times\left(M_{p}^{\mu \alpha}(l)+M_{\Delta}^{\mu \alpha}(l)+\ldots\right)
\end{aligned}
$$

where we introduced the IR-regularization by the photon mass $\lambda$. In what follow we restrict our considerations computing only the elastic contribution $M_{p}^{\mu \alpha}$. This contribution is required for the correct treatment of the QED divergencies in the physical cross section and usually it provides the largest numerical effect. Using (3.11) and

$$
\left\langle p\left|J^{\alpha}(0)\right| p\right\rangle \simeq \bar{N}(p) \gamma^{\alpha} N(p)
$$

we obtain

$$
\begin{aligned}
M_{p}^{\mu \alpha} & \simeq \bar{N}\left(p^{\prime}\right)\left[\left\{\frac{\not h h}{4} \gamma_{\perp}^{\mu} f_{1}(Q)\right\} \frac{i(\not p+m)}{p_{+} l_{-}+i \varepsilon} \gamma^{\alpha}+\gamma^{\alpha} \frac{i\left(\not p^{\prime}+m\right)}{-p_{-}^{\prime} l_{+}+i \varepsilon}\left\{\frac{\text { th }}{4} \gamma_{\perp}^{\mu} f_{1}(Q)\right\}\right] N(p), \\
& \simeq i\left[\frac{\bar{n}^{\alpha}}{l_{-}+i \varepsilon}+\frac{n^{\alpha}}{-l_{+}+i \varepsilon}\right] \bar{N}\left(p^{\prime}\right) \frac{\not h h}{4} \gamma_{\perp}^{\mu} N(p) f_{1}(Q) .
\end{aligned}
$$


Substituting this expression in Eq.(6.14) we obtain

$$
\left\langle p^{\prime}\left|O_{+}^{\mu}\left(Y_{v}^{\dagger} S_{\bar{v}}-1\right)\right| p\right\rangle_{\mathrm{SCET}} \simeq e^{2} \bar{N}\left(p^{\prime}\right) \frac{\not h h}{4} \gamma_{\perp}^{\mu} N(p) f_{1}(Q) J_{e l},
$$

with the scalar integral

$$
J_{e l}=\frac{i e^{2} \mu_{\mathrm{uv}}^{2 \varepsilon}}{(2 \pi)^{D}} \int \frac{d^{D} l}{\left[l^{2}-\lambda^{2}+i \varepsilon\right]}\left\{\frac{\bar{v}^{\alpha}}{[-(\bar{v} l)+i \varepsilon]}+\frac{v^{\alpha}}{[(v l)+i \varepsilon]}\right\}\left\{\frac{\bar{n}^{\alpha}}{(l \bar{n})+i \varepsilon}+\frac{n^{\alpha}}{-(\ln )+i \varepsilon}\right\} .
$$

This integral is the same as the one derived for quarks in Appendix C. This is not surprising because we consider the soft photon exchange between the highly energetic particles (lepton and proton). The effective action describing such a process can be written in the form of the soft photon WLs. The only difference is in the electric charges: the soft photon interacts with the total proton charge. Therefore for the real part we obtain

$$
\operatorname{Re} J_{e l}=-\frac{1}{4 \pi^{2}} \ln \left|\frac{(\bar{v} \bar{n})}{(\bar{v} n)}\right|\left\{\frac{1}{\varepsilon_{\mathrm{uv}}}+\ln \frac{\mu_{\mathrm{uv}}^{2}}{\lambda^{2}}\right\}
$$

where $\mu_{\mathrm{uv}}$ is the UV-renormalization scale in the $\overline{M S}$-scheme. The result can be rewritten as

$$
\ln \left|\frac{(\bar{v} \bar{n})}{(\bar{v} n)}\right| \approx \ln \left|\frac{2(k p)}{2\left(k p^{\prime}\right)}\right|=\ln \left|\frac{s-m^{2}}{-u+m^{2}}\right| \equiv \ln \left|\frac{\tilde{s}}{\tilde{u}}\right|,
$$

where we introduced $\tilde{s}=s-m_{N}^{2}, \tilde{u}=-u+m^{2}$. After UV-renormalization we find

$$
\left\langle p^{\prime}\left|O_{+}^{\mu}\left(Y_{v}^{\dagger} S_{\bar{v}}-1\right)\right| p\right\rangle_{\mathrm{SCET}}=\frac{\alpha}{\pi} \bar{N}\left(p^{\prime}\right) \frac{\not h \phi}{4} \gamma_{\perp}^{\mu} N(p) f_{1}(Q) \ln \frac{\lambda^{2}}{\mu_{\mathrm{uv}}^{2}} \ln \left|\frac{\tilde{s}}{\tilde{u}}\right| .
$$

We will make a few comments on this result. The UV-divergence arises as soon as we neglect the small terms $l^{2}$ in the denominators of the nucleon in Eq.(6.12). Such approximation can be understood as a transition to the effective theory which describes the interaction of the energetic particles ${ }^{7}$ with the soft photons. The appropriate effective action can be naturally described by the soft photon WLs directed along the proton momenta. Formally the UV-divergence is related to the multiplicative renormalization of WLs. The UV-renormalization induces the dependence on the scale $\mu_{\mathrm{uv}}$ which we can be understood as highest possible virtuality of the soft photon consistent with our approximations. It is naturally to expect that we can put $\mu_{\mathrm{uv}}=\mu_{0} \sim \Lambda$. In the final expression (6.21) we keep the exact expression $\ln \left|\frac{\tilde{s}}{\tilde{u}}\right|$ because this expression provides the exact cancellation of the IR-divergent contribution. This point is discussed in detail in the next section.

Our calculation has many common features with the soft approximation made in Ref.[10]. In that work the TPE diagram with the nucleon intermediate state has also been computed neglecting the small photon momentum $l$ in the numerator of the integrand (including the FF of the hard vertex) and in the hard photon propagator but not in the denominators of the electron and proton lines. As a result, the integrand is reduced

\footnotetext{
${ }^{7}$ Remind that in our frame the leptons and protons move with the energies of order $s \sim Q$.
} 
to a three-point function. The resulting integral is UV-finite but the answer unavoidably includes the region where the virtuality $l^{2}$ of the photon (and other particles) can be large $l^{2} \sim Q^{2}$. But such description of this hard region is incorrect because the dynamics of the hard particles must be described using QCD. As a result the result of Refs. [10, 39] includes large logarithms $\ln \lambda^{2} / s$

$$
\bar{\delta}^{2 \gamma}[\text { Tsai }] \sim \ln \frac{\lambda^{2}}{s} \ln \left|\frac{\tilde{s}}{\tilde{u}}\right| G_{M}^{2}+\ldots .
$$

because in this case the hard scale $s$ provides the required "UV cut-off". Therefore we expect that such description of the TPE contribution in the region of large energies is incorrect in spite of the fact that it correctly treats the QED IR behavior.

In our calculation we should not have any region corresponding with hard virtualities because we already factorized the hard dynamics. Therefore it is important to perform the expansion in small momenta $l$ as in (6.12) in order to avoid the double counting of the hard region. Then we do not obtain any large logarithms. The uncertainty in the choice of the renormalization scale $\mu_{\mathrm{uv}} \sim \mu_{0}$ has a clear physical meaning and is associated with the applicability of the our soft approximation and also reflects an ambiguity in the calculation of the initial condition $g_{1}\left(z, \mu_{0}\right)$.

Hence assuming the dominance of the elastic contribution and comparing Eq.(6.21) with (3.31) we obtain

$$
g_{1}\left(z, Q, \mu_{0}\right) \simeq \frac{\alpha}{\pi} f_{1}(Q) \ln \frac{\mu_{0}^{2}}{\lambda^{2}} \ln \bar{z} \simeq \frac{\alpha}{\pi} G_{M}\left(Q^{2}\right) \ln \frac{\lambda^{2}}{\mu_{0}^{2}} \ln \left|\frac{\tilde{s}}{\tilde{\tilde{u}}}\right|, \quad g_{3}(z, Q) \simeq 0 .
$$

where we used Eq.(3.15) to re-express the SCET FF $f_{1}$ in term of the physical FF $G_{M}$.

We obtained that the second SCET amplitude $g_{3}$ in this approximation is very small. We suppose that this is a feature of our model in which the soft photon interacts with the hadron as with a structureless point-like particle. We expect that contributions with higher spins in Eq.(6.14) also do not provide any significant contributions to $g_{3}$. Probably, this amplitude is sensitive to the region with the relatively higher virtualities for which the soft photon can still resolve the soft and hard-collinear structures, i.e. where the soft photon is sensitive to the rapidity gap arising due to the soft spectator scattering.

\section{Phenomenological analysis}

In order to make a numerical analysis we eliminate the SCET FF $\mathcal{F}_{1}$ using the expression in Eq.(5.15). Consider for simplicity the amplitude $\tilde{F}_{3}$. Using the soft spectator contributions given in Eq.(4.18) we obtain

$$
\frac{\nu}{m^{2}} \tilde{F}_{3}\left(\varepsilon, Q^{2}\right)=g_{3}(z, Q)+\frac{\alpha}{\pi} \frac{\nu}{s} C_{3}(s, t) \mathcal{F}_{1}+\mathbf{\Psi}\left(x_{i}\right) * H_{3}\left(z, Q^{2}, x_{i}, y_{i}\right) * \mathbf{\Psi}\left(y_{i}\right),
$$

where for the hard spectator scattering contribution we used expressions given in Eqs.(4.15). Substituting Eq.(5.15) for $\mathcal{F}_{1}$ into Eqs.(7.1) (we assume the different value of $s=s^{\prime}$ for 
WACS) we obtain

$$
\begin{aligned}
\frac{\nu}{m^{2}} \tilde{F}_{3}\left(\varepsilon, Q^{2}\right)= & g_{3}(z, Q)+\frac{\alpha}{\pi} \frac{\nu}{s} C_{3}(s, t) \frac{T_{2}\left(s^{\prime}, t\right)}{C_{2}\left(s^{\prime}, t\right)} \\
& +\boldsymbol{\Psi}\left(y_{i}\right) *\left\{H_{3}\left(z, Q^{2} ; x_{i}, y_{i}\right)-\frac{H_{2}\left(s^{\prime}, t ; x_{i}, y_{i}\right)}{C_{2}\left(s^{\prime}, t\right)}\right\} * \boldsymbol{\Psi}\left(x_{i}\right), \\
\simeq & \frac{\alpha}{\pi} \frac{\nu}{s} C_{3}(s, t) \mathcal{R}(Q)+\boldsymbol{\Psi}\left(y_{i}\right) * H_{3}\left(z, Q^{2} ; x_{i}, y_{i}\right) * \boldsymbol{\Psi}\left(x_{i}\right) .
\end{aligned}
$$

In order to obtain the last equation we used the estimate (6.23) in which $g_{3}$ is zero, substituted $T_{2}\left(s^{\prime}, t\right) / C_{2}\left(s^{\prime}, t\right)=\mathcal{R}\left(s^{\prime}, t\right) \simeq \mathcal{R}(Q)$ and we also neglected the contributions of order $\mathcal{O}\left(\alpha_{s}^{2}\right)$ in the hard coefficient functions $H_{2,3}$, keeping in mind that the leading order contributions are $H_{3, M} \sim \mathcal{O}\left(\alpha_{s}\right)$ while $H_{2} \sim \mathcal{O}\left(\alpha_{s}^{2}\right)$ and $C_{2} \sim \mathcal{O}\left(\alpha_{s}^{0}\right)$. An analogous expression can be also obtained for the TPE amplitude $\delta \tilde{G}_{M}^{2 \gamma}$ and reads

$$
\begin{aligned}
\delta \tilde{G}_{M}^{2 \gamma}\left(\varepsilon, Q^{2}\right) \simeq \frac{\alpha}{\pi} G_{M}\left(Q^{2}\right) \ln \frac{\lambda^{2}}{\mu_{0}^{2}} \ln \left|\frac{s-m^{2}}{u-m^{2}}\right|+\frac{\alpha}{\pi} C_{M}\left(z, Q^{2}, \mu_{0}\right) \mathcal{R}(Q) \\
+\boldsymbol{\Psi}\left(y_{i}\right) * H_{M}\left(z, Q^{2} ; x_{i}, y_{i}\right) * \mathbf{\Psi}\left(x_{i}\right) .
\end{aligned}
$$

where we used Eq.(6.23) for $g_{1}$ and assumed that $H_{M} \sim \mathcal{O}\left(\alpha_{s}\right)$.

The formulas presented in Eqs. $(7.3,7.4)$ will be used for the numerical estimates presented below. We like to emphasize that these expressions are of course model dependent. We expect that the largest ambiguity arises from the amplitudes $g_{1,3}$ which were computed in the hadronic model in Eqs. (6.23), and which describe the TPE subprocess with one soft photon.

The other terms in Eqs. (7.3,7.4) describe the contributions with two hard photons and can be fixed more accurately. For the ratio $\mathcal{R}$ we use the fit (5.31) with the parameters $c=0.10 \pm 0.01$ and $\Lambda=1.08 \pm 0.03 \mathrm{GeV}$. In the numerical calculations we use for the scale $\mu_{0}=1 / \sqrt{-t}$ yielding $\mu_{0}=630-450 \mathrm{MeV}$ for $Q^{2}=2.5-5 \mathrm{GeV}^{2}$. We observed that variations of the value of this scale by $\pm 100 \mathrm{MeV}$ do not provide any significant numerical effect. The explicit expressions for the coefficient functions $H_{M, 3}$ and distribution amplitudes $\boldsymbol{\Psi}$ can be found in Ref. [32, 33]. The DA $\boldsymbol{\Psi}$ can be expressed in terms of one scalar DA for which we use the model

$$
\varphi_{N}\left(x_{1}, x_{2}, x_{3}\right)=f_{N} 120 x_{1} x_{2} x_{3}\left\{1+r_{+}\left(1-3 x_{3}\right)+r_{-}\left(x_{2}-x_{1}\right)\right\},
$$

where the low energy constants $f_{N}, r_{+}$and $r_{-}$have been estimated at $\mu=1 \mathrm{GeV}$ as [30]:

$$
f_{N}=(5.0 \pm 0.5) \times 10^{-3} \mathrm{GeV}^{2}, r_{+} \simeq 0.35, r_{-} \simeq 1.37
$$

The scale of the running coupling $\alpha_{s}\left(\mu_{R}^{2}\right)$ was set to be $\mu_{R}^{2} \simeq 0.6 Q^{2}$ and we use the two-loop running coupling with $\alpha_{s}\left(\mu_{R}^{2}=1.5 \mathrm{GeV}^{2}\right)=0.360$.

In our numerical calculations we also use the ratio $G_{E} / G_{M}$ which is fixed from the experimental data [1-5] and using the following fit of the data for $Q^{2}$ in the interval $0.5-8.5 \mathrm{GeV}^{2}$ :

$$
R \equiv \frac{G_{E}}{G_{M}}=\mu_{p}^{-1}\left(1-a \ln ^{2}\left[Q^{2} / \Lambda_{R}^{2}\right]\right)
$$


with $a=0.070 \pm 0.007, \Lambda_{R}=0.54 \pm 0.03 \mathrm{GeV}$. The other parameters which we need are

$$
m=0.938 \mathrm{GeV}, \mu_{p}=2.7928, \alpha=1 / 137 .
$$

Finally let us inspect the behavior of the amplitudes $(7.3,7.4)$ in the limit $\varepsilon \rightarrow 1(z \rightarrow 0)$ where TPE corrections must be consistent with behavior Eq.(2.14). Using the expressions for $C_{M, 3}$ given in Eqs.(4.9,4.10), we obtain in this limit :

$$
\delta \tilde{G}_{M}^{2 \gamma}\left(\varepsilon=1, Q^{2}\right)=-\frac{\nu}{m^{2}} \tilde{F}_{3}\left(\varepsilon=1, Q^{2}\right)=\frac{\alpha \pi}{2} \mathcal{F}_{1}(Q),
$$

where we used that hard spectator contributions vanish separately at $\varepsilon=1$

$$
\lim _{z \rightarrow 0} \mathbf{\Psi}\left(y_{i}\right) * H_{M, 3}\left(z, Q^{2} ; x_{i}, y_{i}\right) * \mathbf{\Psi}\left(x_{i}\right)=0 .
$$

This can be easily shown using the explicit expressions from Ref.[32, 33]. Using Eqs.(7.9) one finds that Eq.(2.14) is fulfilled.

\subsection{Reduced cross section}

The formula for the elastic reduced cross section presented in Eq.(2.20) can not be compared to the observable cross section because of IR-divergent amplitudes. For the real parts, the IR divergence arising from the elastic next-to-leading (NLO) QED diagrams is canceled by IR-divergent contributions arising from the interference of the bremsstrahlung and Born diagrams. Therefore one must include also the contribution of the inelastic diagrams. After that the reduced cross section can be presented as following

$$
\sigma_{R}^{\exp }=\sigma_{R}^{1 \gamma}\left(1+\delta_{\mathrm{el}, \mathrm{virt}}+\delta_{\mathrm{brems}}\right)
$$

where the Born cross section reads

$$
\sigma_{R}^{1 \gamma}=G_{M}^{2}+\frac{\varepsilon}{\tau} G_{E}^{2}
$$

The contribution of the virtual (real) radiative corrections to the elastic amplitudes in Eq.(7.11) is included in $\delta_{e l, \text { virt }}\left(\delta_{\text {brems }}\right)$ respectively. The analysis of the radiative corrections for the existing experimental data has been carried out using the Mo and Tsai (MT) formulas [10]. Let us write in this case the reduced cross section as

$$
\sigma_{R}^{\exp }=\sigma_{R}^{1 \gamma, \mathrm{MT}}\left(1+\delta_{\mathrm{virt}}^{\mathrm{MT}}+\delta_{\mathrm{brems}}^{\mathrm{MT}}\right)
$$

In this case the extracted values of $\sigma_{R}^{1 \gamma, \mathrm{MT}}$ can be considered as experimental results. If one uses a different approach to compute the radiative corrections with a different elastic input then the extracted value of the Born cross section is different. This difference must be considered as effect related to the TPE correction in our case. In what follows we use the same expressions for the elastic contributions other than two-photon exchange, and for the bremsstrahlung contribution as in MT-formulas, i.e.

$$
\begin{aligned}
\delta_{e l, \mathrm{virt}}-\delta_{e l, \mathrm{virt}}^{\mathrm{MT}} & =\delta_{2 \gamma}-\delta_{2 \gamma}^{\mathrm{MT}} \\
\delta_{\text {brems }} & =\delta_{\text {brems }}^{\mathrm{MT}}
\end{aligned}
$$


Therefore from Eq.(7.11) and Eq.(7.13) we obtain

$$
\sigma_{R}^{1 \gamma, \mathrm{MT}}=\sigma_{R}^{1 \gamma}\left(1+\delta_{2 \gamma}-\delta_{2 \gamma}^{\mathrm{MT}}\right) .
$$

where the FFs $G_{M}$ and $G_{E}$ on the $r h s$ must be considered as unknown quantities. Using Eq.(2.20) one can write the explicit expressions for the TPE contributions as

$$
\sigma_{R}^{1 \gamma} \delta_{2 \gamma}=2 G_{M} \operatorname{Re}\left[\delta \tilde{G}_{M}^{2 \gamma}+\varepsilon \frac{\nu}{m^{2}} \tilde{F}_{3}\right]+2 \frac{\varepsilon}{\tau} G_{E} \operatorname{Re}\left[\delta \tilde{G}_{E}^{2 \gamma}+\frac{\nu}{m^{2}} \tilde{F}_{3}\right] .
$$

It is convenient to present the analytical expression for the MT term $\delta_{2 \gamma}^{\mathrm{MT}}$ in the similar form. Using the analytical expression presented in [39] we can write

$$
\sigma_{R}^{1 \gamma} \delta_{2 \gamma}^{\mathrm{MT}}=2 G_{M} \operatorname{Re}\left[G_{M} \frac{1}{2} \delta_{2 \gamma}^{\mathrm{MT}}\right]+2 \frac{\varepsilon}{\tau} G_{E} \operatorname{Re}\left[G_{E} \frac{1}{2} \delta_{2 \gamma}^{\mathrm{MT}}\right]
$$

The analytical expression for the factor $\delta_{2 \gamma}^{\mathrm{MT}}$ reads

$$
\operatorname{Re} \delta_{2 \gamma}^{\mathrm{MT}}=\frac{2 \alpha}{\pi}\left\{\ln \frac{\lambda^{2}}{\tilde{s}} \ln \left|\frac{\tilde{s}}{\tilde{u}}\right|+\frac{1}{2} \ln ^{2}\left|\frac{\tilde{s}}{\tilde{u}}\right|-\operatorname{Li}\left(\frac{\tilde{s}}{s}\right)-\frac{1}{2} \ln ^{2} \frac{\tilde{s}}{s}+\operatorname{Re} \operatorname{Li}\left(\frac{\tilde{u}}{u}\right)+\frac{1}{2} \ln ^{2} \frac{\tilde{u}}{u}\right\},
$$

where $\lambda^{2}$ is the soft photon mass (IR regulator), $\tilde{s}=s-m^{2}, \tilde{u}=u-m^{2}$ and $\operatorname{Li}(z)$ is the Spence function (dilogarithm) defined by

$$
\operatorname{Li}(z)=-\int_{0}^{z} d t \frac{\ln (1-t)}{t} .
$$

The result presented in Eq.(7.18) is obtained by computing the box diagrams with nucleon intermediate state in the soft limit [10]. However the expression in Eq.(7.18) is incomplete because the exact answer has one more term [39]

$$
\left[\delta_{2 \gamma}^{\mathrm{MT}}\right]_{\text {exact }}=\delta_{2 \gamma}^{\mathrm{MT}}+\alpha \pi
$$

However the factor $\alpha \pi$, which originates from the crossed box diagram ${ }^{8}$, is absent in the formulas in Ref. [10] and therefore is excluded from the expression of $\delta_{2 \gamma}^{\mathrm{MT}}$.

Using Eqs.(7.16), (7.17) we can present the rhs in Eq.(7.15) as

$$
\begin{aligned}
\sigma_{R}^{1 \gamma, \mathrm{MT}}=\sigma_{R}^{1 \gamma} & +2 G_{M} \operatorname{Re}\left[\delta \tilde{G}_{M}^{2 \gamma}+\varepsilon \frac{\nu}{m^{2}} \tilde{F}_{3}-G_{M} \frac{1}{2} \delta_{2 \gamma}^{\mathrm{MT}}\right] \\
& +2 \frac{\varepsilon}{\tau} G_{E} \operatorname{Re}\left[\delta \tilde{G}_{E}^{2 \gamma}+\frac{\nu}{m^{2}} \tilde{F}_{3}-G_{E} \frac{1}{2} \delta_{2 \gamma}^{\mathrm{MT}}\right] .
\end{aligned}
$$

The amplitudes $\delta \tilde{G}_{M}^{2 \gamma}, \delta \tilde{G}_{E}^{2 \gamma}$ and $\delta_{2 \gamma}^{\mathrm{MT}}$ in Eq.(7.21) depend on the IR photon mass $\lambda^{2}$ which however cancels in the difference, we discuss these subject in detail in Appendix E.

The TPE contribution associated with the $\delta \tilde{G}_{E}^{2 \gamma}$ in Eq.(7.21)

$$
\delta \tilde{G}_{E}^{2 \gamma}+\frac{\nu}{m^{2}} \tilde{F}_{3}=\delta \tilde{G}_{M}^{2 \gamma}-(1+\tau) \delta \tilde{F}_{2}+\frac{\nu}{m} \tilde{F}_{3},
$$

\footnotetext{
${ }^{8}$ More explicitly, the factor $\alpha \pi$ in Eq.(7.20) originates from analytic continuation of $\ln ^{2}$ terms when evaluating crossed box diagram from the direct box diagram as shown in Ref.[50]
} 
contains the unknown amplitude $\delta \tilde{F}_{2}$. This amplitude depends on the matrix elements of subleading SCET operators which we did not consider in our analysis. We will assume that the corresponding difference $\delta \tilde{G}_{E}^{2 \gamma}+\frac{\nu}{m^{2}} \tilde{F}_{3}-G_{E} \frac{1}{2} \delta_{2 \gamma}^{\mathrm{MT}}$ in Eq.(7.21) behaves similar to the FF $G_{E}=G_{M}-(1+\tau) F_{2}=R G_{M}$ and therefore

$$
\delta \tilde{G}_{E}^{2 \gamma}+\frac{\nu}{m^{2}} \tilde{F}_{3}-G_{E} \frac{1}{2} \delta_{2 \gamma}^{\mathrm{MT}} \sim \frac{\alpha}{\pi} \mathcal{O}(R)
$$

In this case the correction originating from such term in Eq.(7.21) is relatively small

$$
2 \frac{\varepsilon}{\tau} G_{E} \operatorname{Re}\left[\delta \tilde{G}_{E}^{2 \gamma}+\frac{\nu}{m^{2}} \tilde{F}_{3}-G_{E} \delta_{2 \gamma}^{\mathrm{MT}}\right] \sim 2 \frac{\varepsilon}{\tau} G_{M}^{2} \frac{\alpha}{\pi} \times \mathcal{O}\left(R^{2}\right),
$$

because the ratio $R$ is small and we can neglect it. Therefore in our numerical analysis we use for the reduced cross section the following expression

$$
\sigma_{R}^{1 \gamma, \mathrm{MT}}=G_{M}^{2}+\frac{\varepsilon}{\tau} G_{E}^{2}+2 G_{M} \operatorname{Re}\left[\delta \tilde{G}_{M}^{2 \gamma}+\varepsilon \frac{\nu}{m^{2}} \tilde{F}_{3}-G_{M} \frac{1}{2} \delta_{2 \gamma}^{\mathrm{MT}}\right] .
$$

The values $\sigma_{R}^{1 \gamma, \mathrm{MT}}$ must be considered as experimental data. Taking into account that the ratio $R=G_{E} / G_{M}$ is also measured, see Eq.(7.7), one finds that only the FF $G_{M}$ is an unknown quantity on the $r h s$ of the Eq.(7.25). Therefore using Eq.(7.25) we can extract the value $G_{M}$ using the data from the kinematical region where our approximation is valid: $s \sim-t \sim-u \gg \Lambda$. We use the data from Refs. [7, 9]. In Figs.13,14 we show the fit of the experimental data for different values of $Q$. The shaded area shows a transition region where the Mandelstam variable $u$ is already quite small and one may expect sizable contributions originating from the higher order and, probably, power corrections. The
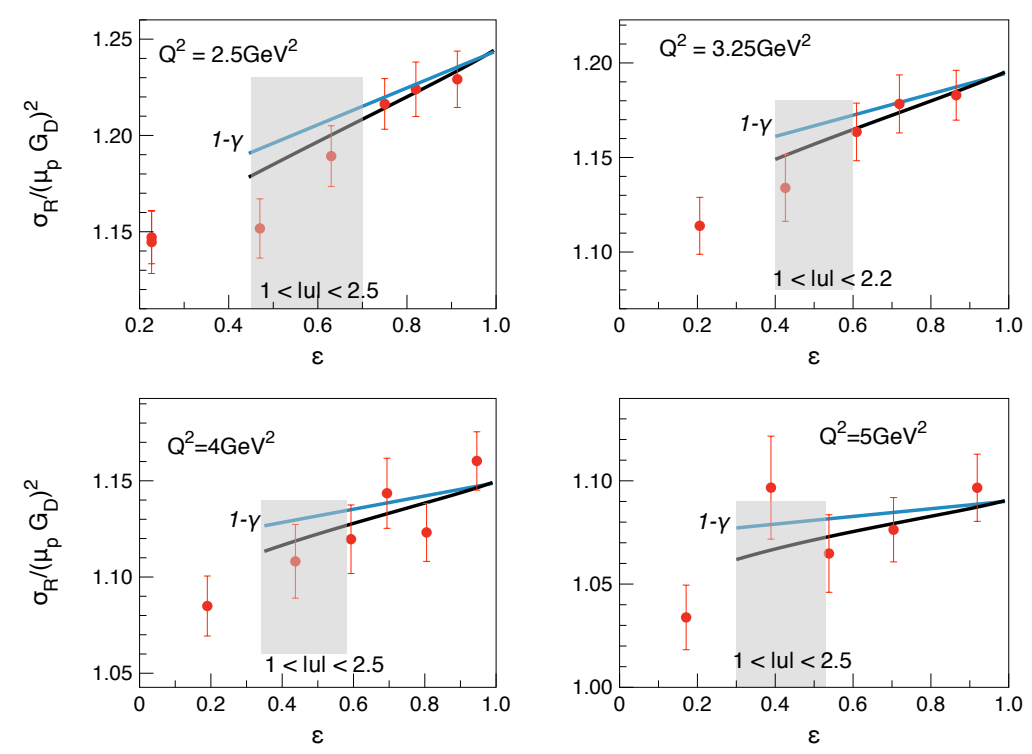

Figure 13. The fit of data for the $e p \rightarrow e p$ elastic scattering reduced cross section from [7] for different values of $Q^{2}$. For the fit we used only the data points which are lying on the right of the shaded area. 

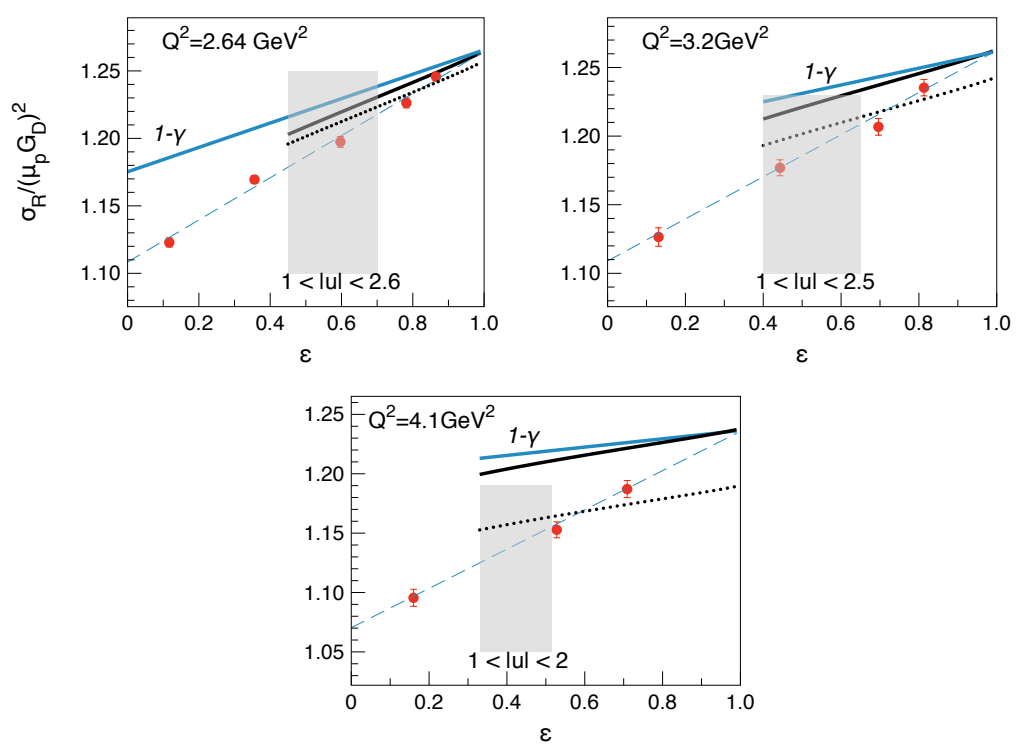

Figure 14. The fit of $G_{M}$ using the data [9] with the computed TPE corrections. The black solid line shows the $\sigma_{R}$ with $G_{M}^{[\text {Gut }]}$ extracted from the linear fit in [66]. The black dotted curve represents the fit of the data points which are lying on the right of the shaded area.

corresponding values of $|u|$ (in $\mathrm{GeV}^{2}$ ) are shown at the bottom of the plot. For the fit we used only the points which are lying on the right of the shaded area. First we observe that the obtained TPE contribution is relatively small. It turns out that the soft spectator contribution by coincidence is numerically very close to the MT result for the considered range of $Q^{2}$.

As we can see from the plots the obtained behavior of $\sigma_{R}(\varepsilon)$ (solid black line) is linear in the region of $\varepsilon$ where the calculation can be applied and describes the difference in the slope between polarized and unpolarized data quite reasonably for the SLAC data within the relatively large error bars. The comparison is worse for the JLab data. However for the JLab data we have to take into account that, for instance, for $Q^{2}=4.1 \mathrm{GeV}^{2}$ the second point corresponds already to relatively low values of $u$.

The main trend is that in the region of small $\varepsilon$ the slope must change and this means that the reduced cross section can not be described by the linear function in $\varepsilon$ at large $Q$. Such nonlinearity is still relatively small at $Q^{2}=2.64 \mathrm{GeV}^{2}$ (the linear fit is shown by the blue dashed line in Fig.14 ). But at $Q^{2}=4.1 \mathrm{GeV}^{2}$ this effect might already be sizable. Such a scenario implies a large corrections when $-u \rightarrow 1 \mathrm{GeV}^{2}$, i.e. in the backward region. Theoretically it may be interesting to go beyond the formalism presented in this work and compute the TPE corrections using QCD factorization in the region with small $|u| \lesssim m^{2}$ in order to have description for the whole region of $\varepsilon$.

The extracted values of $G_{M}$ are shown in Table 1 and for comparison we also provide the results of different phenomenological fits made in Refs. [66-68]. 
Table 1. Results for magnetic form factor obtained in this work from the fit of SLAC data [7] (black solid line in Fig.13) and JLab data [9] (dotted curve in Fig.14). The extracted values denoted as $G_{M}^{[\text {fact }]}$. For comparison we show the phenomenological extractions presented in Refs. [66] $\left(G_{M}^{[\mathrm{Gut}]}\right)$, [67] $\left(G_{M}^{[\mathrm{Br}]}\right)$ and $[68]\left(G_{M}^{[\mathrm{Ven}]}\right)$

\begin{tabular}{|c|c|c|c|c|}
\hline$Q^{2}, \mathrm{GeV}^{2}$ & $G_{M}^{[\mathrm{fact}]}$ & $G_{M}^{[\mathrm{Gut}]}$ & $G_{M}^{[\mathrm{Br}]}$ & $G_{M}^{[\mathrm{Ven}]}$ \\
\hline \multicolumn{5}{|c|}{ JLab } \\
\hline 2.64 & 0.1356 & 0.1360 & 0.1350 & 0.1352 \\
\hline 3.2 & 0.1001 & 0.1009 & 0.0985 & 0.0989 \\
\hline 4.1 & 0.0654 & 0.0667 & 0.0641 & 0.0647 \\
\hline \multicolumn{5}{|c|}{ SLAC } \\
\hline 2.5 & 0.1464 & - & 0.1471 & 0.1472 \\
\hline 3.25 & 0.0958 & - & 0.0960 & 0.0963 \\
\hline 4.0 & 0.0670 & - & 0.0670 & 0.0675 \\
\hline 5.0 & 0.0447 & - & 0.0446 & 0.0452 \\
\hline
\end{tabular}

In Fig.15 we show the TPE amplitudes which are defined as

$$
Y_{M}=\left(\delta \tilde{G}_{M}^{2 \gamma}-G_{M} \frac{1}{2} \delta_{2 \gamma}^{\mathrm{MT}}\right) / G_{M}, Y_{3}=\delta \tilde{F}_{3} / G_{M}
$$

These ratios are plotted for three fixed values of $Q^{2}=2.64,3.2,4.1 \mathrm{GeV}^{2}$ as a function of $\varepsilon$. For the FF $G_{M}$ we take the values shown in Table 1. As we can see these functions show a weak dependence on $Q^{2}$. At $Q^{2}=2.64 \mathrm{GeV}^{2}$ these functions have been estimated

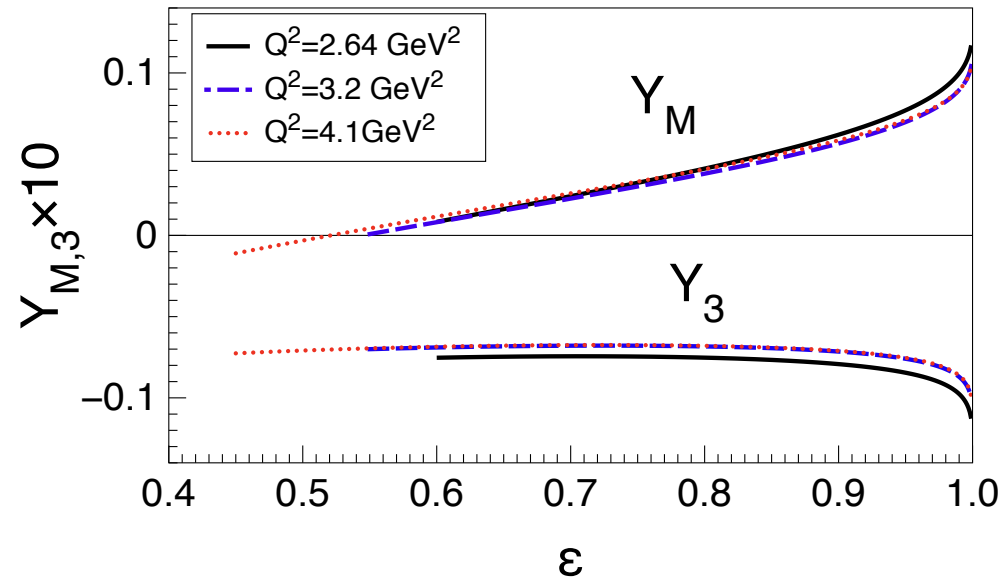

Figure 15. TPE amplitudes (7.26) as a functions of $\varepsilon$ at fixed values of $Q^{2}$.

from the data using certain assumption about their behavior at $\varepsilon=1$, see e.g. [66, 69, 70]. Thus the present calculation shows that the assumption that the amplitudes $Y_{M, 3}$ vanish 
separately at $\varepsilon \rightarrow 1$ is probably too strong. The absolute values of the $Y_{M, 3}$ in Fig.15 are also much smaller than the phenomenological ones shown in [66].

The other good observable to access the TPE corrections is to measure the ratio of the elastic $e^{ \pm} p$ cross sections

$$
R_{ \pm}=\frac{d \sigma_{e^{+} p}}{d \sigma_{e^{-} p}}
$$

If the corresponding reduced cross section $\sigma_{R}^{1 \gamma}$ extracted with MT radiative corrections [10] are incomplete then this ratio will differ from unity due to the TPE contribution. In our case using Eq.(7.25) we find

$$
\begin{aligned}
R_{ \pm} & \simeq \frac{\sigma_{R}^{1 \gamma}-2 G_{M} \operatorname{Re}\left[\delta \tilde{G}_{M}^{2 \gamma}+\varepsilon \frac{\nu}{m^{2}} \tilde{F}_{3}-G_{M} \frac{1}{2} \delta_{2 \gamma}^{\mathrm{MT}}\right]}{\sigma_{R}^{1 \gamma}+2 G_{M} \operatorname{Re}\left[\delta \tilde{G}_{M}^{2 \gamma}+\varepsilon \frac{\nu}{m^{2}} \tilde{F}_{3}-G_{M} \frac{1}{2} \delta_{2 \gamma}^{\mathrm{MT}}\right]} \\
& \approx 1-\frac{4 G_{M}}{\sigma_{R}^{1 \gamma}} \operatorname{Re}\left[\delta \tilde{G}_{M}^{2 \gamma}+\varepsilon \frac{\nu}{m^{2}} \tilde{F}_{3}-G_{M} \frac{1}{2} \delta_{2 \gamma}^{\mathrm{MT}}\right]
\end{aligned}
$$

Using the values $G_{M}$ obtained from the analysis of the JLab data we obtain the ratio $R_{ \pm}$ shown in Fig.16 as a function of $\varepsilon$ for three different values of $Q^{2}=2.64,3.2$ and $4.1 \mathrm{GeV}^{2}$, respectively. For comparison we also show the predictions made in [66] on the basis of linear fit for the same values of momentum transfer. Because we obtained a relative large difference between the slopes for the reduced cross section we also obtain a large difference in the estimate of the ratio (7.29). It is interesting that the absolute value of the obtained TPE correction within the factorization formalism changes very slowly with respect to $Q^{2}$.

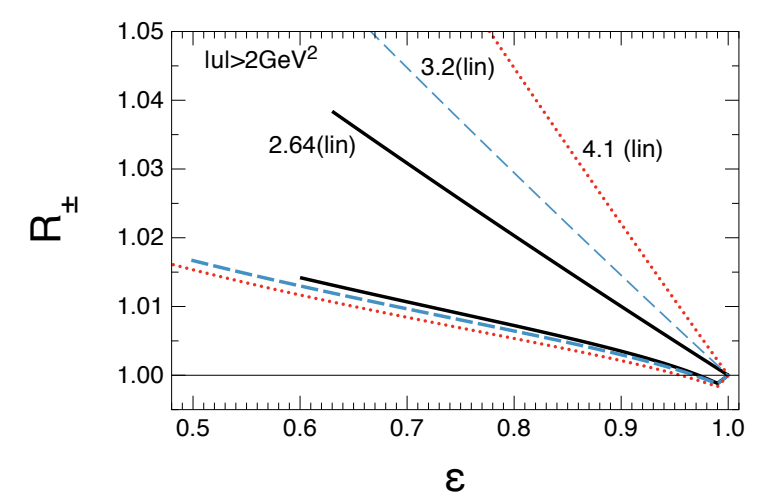

Figure 16. The ratio $R_{ \pm}$for different values of $Q^{2}$ (in $\mathrm{GeV}^{2}$ ): $Q^{2}=2.64$ (black solid curve), $Q^{2}=3.2$ (blue dashed curve) and $Q^{2}=4.1$ ( red dotted curve). For comparison we also show the results for the linear fit obtained in [66]. The value of $\varepsilon$ are restricted to the range $|u|>2 \mathrm{GeV}^{2}$.

Let us stress that the above results obtained within the simple model of the SCET amplitudes $g_{1,3}$, see Eq.(6.23). One cannot exclude that this rough model underestimates the contribution of the amplitudes $g_{1,3}$. Nevertheless the above calculations clearly show that the TPE contribution associated with two hard photons cannot describe the reduced cross section at large $Q^{2}$ being consistent with the assumption about the linear behavior of 
$\sigma_{R}(\varepsilon)$ in the whole interval $0<\varepsilon<1$ at large fixed $Q^{2}$. Therefore, in order to understand the TPE contribution better it is very important to obtain a realistic estimate of the amplitudes $g_{1,3}$. It turns out that the important information about the amplitude $g_{3}$ can be obtained from the analysis of polarization observables.

\subsection{Recoil polarization observables}

The GEp- $2 \gamma$ collaboration has measured the $\varepsilon$-dependence of the recoil polarizations at a fixed value of $Q^{2}=2.64 \mathrm{GeV}^{2}[6]$. These data provide additional important constraints on the theoretical analysis of the TPE amplitudes.

Let us to start the discussion with the longitudinal polarization $P_{l}$ which in the elastic approximation is given in Eq.(2.22). Taking into account the inelastic contributions and assuming that radiative corrections for the experimental data have been performed according to MT formulas we can follow the same line as for the reduced cross section. Introducing the new FFs $G_{M}$ and $G_{E}$ and expanding the reduced cross section we obtain

$$
\begin{gathered}
P_{l} / P_{l}^{\text {Born }}=1-\frac{2 \varepsilon}{1+\frac{\varepsilon}{\tau} R^{2}} \frac{1}{G_{M}}\left\{\frac{\varepsilon}{1+\varepsilon} \frac{\nu}{m^{2}} \tilde{F}_{3}+\frac{R}{\tau}\left(\delta \tilde{G}_{E}+\frac{\nu}{m^{2}} \tilde{F}_{3}-G_{E} \frac{1}{2} \delta_{2 \gamma}^{\mathrm{MT}}\right)\right. \\
\left.-\frac{1}{\tau} R^{2}\left(\frac{\varepsilon}{1+\varepsilon} \frac{\nu}{m^{2}} \tilde{F}_{3}+\delta \tilde{G}_{M}-G_{M} \frac{1}{2} \delta_{2 \gamma}^{\mathrm{MT}}\right)\right\} .
\end{gathered}
$$

Using Eqs.(2.14,7.9) and Eqs.(5.15,5.20) one can easily find that in the forward limit $\varepsilon \rightarrow 1$ this asymmetry does not vanish

$$
P_{l} / P_{l}^{B o r n}(\varepsilon=1) \simeq 1+\frac{1}{1+\frac{1}{\tau} R^{2}} \frac{\alpha \pi}{2} \frac{\mathcal{F}_{1}(Q)}{G_{M}} \simeq 1+\frac{\alpha \pi}{2} \frac{\mathcal{R}(Q)}{G_{M}}
$$

Taking into account the relative smallness of $\mathcal{O}\left(R^{2}\right)$-contributions and our assumption (7.24) we can conclude that the ratio in Eq.(7.30) is dominated by the first term $\sim \tilde{F}_{3}$ in the brackets and can be approximated as

$$
P_{l} / P_{l}^{\text {Born }} \simeq 1-\frac{2 \varepsilon^{2}}{1+\varepsilon} \frac{\frac{\nu}{m^{2}} \tilde{F}_{3}}{G_{M}} .
$$

Taking into account that $\tilde{F}_{3}$ is IR-finite and $\tilde{F}_{3}\left(Q^{2}, \varepsilon\right)=0$ in the MT-calculation we can use $P_{l}$ in order to extract information about the amplitude $\tilde{F}_{3}$. The kinematical factor $\varepsilon^{2}$ appearing in Eq.(7.32) reduces the possibility of such analysis only to the region of relatively large values of $\varepsilon$ where our approximation is valid. In Fig.17 we demonstrate our estimates for the $P_{l} / P_{l}^{\text {Born }}$ as a function of $\varepsilon$ for different values of $Q^{2}$. It turns out that the hard spectator contribution $\tilde{F}_{3}^{(h)}$ is quite small (red dotted line in left plot ) compared to the soft spectator contribution $\tilde{F}_{3}^{(s)}$. From Fig.17 we also see that the dependence on $Q$ is rather weak. However this function has a steep behavior in $\varepsilon$ in the vicinity of $\varepsilon=1$. Such behavior originates from the $\varepsilon$-dependence of the hard coefficient function $C_{3}(z)$, see Eq.(4.10). On the right plot in Fig.17 we show a comparison of our estimate at $Q^{2}=2.64 \mathrm{GeV}^{2}$ with the existing experimental data [6]. For comparison we show the solid and dashed curves corresponding to the two different fits for $\mathcal{F}_{1}$ : with and without 

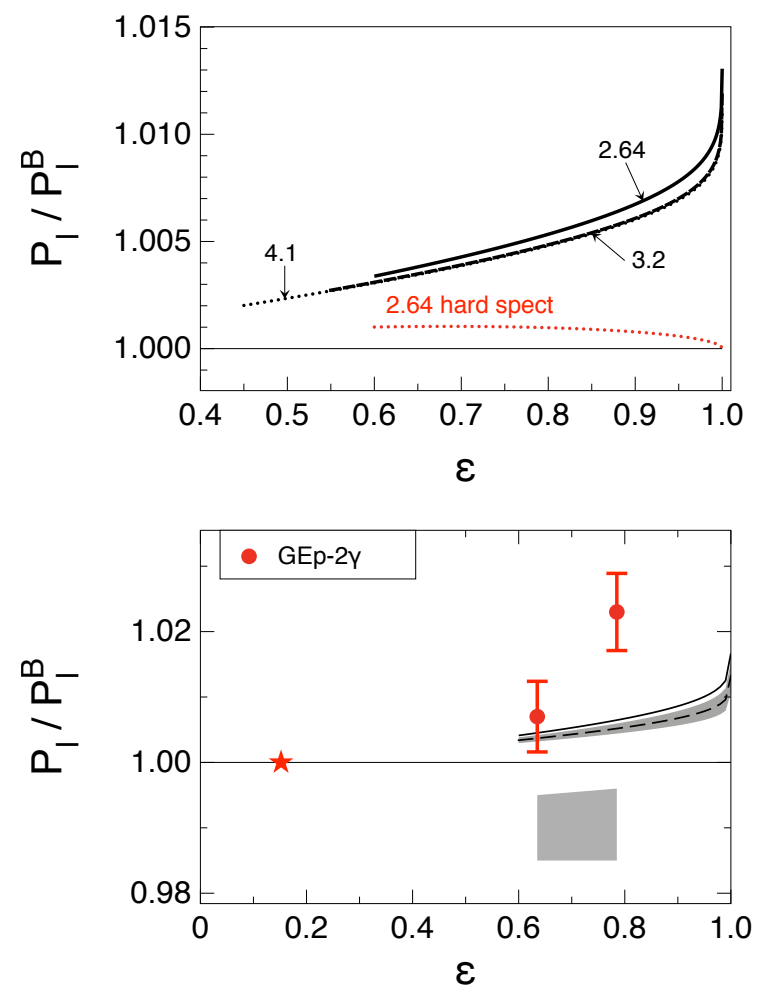

Figure 17. The ratio $P_{l} / P_{l}^{\text {Born }}$ as a function of $\varepsilon$ for different values of $Q$ (left) and comparison of our estimates with experimental data [6] at $Q^{2}=2.64 \mathrm{GeV}^{2}$. The shaded area around the dashed line demonstrates the ambiguity arising from $\mathcal{R}$. The solid line shows the ratio where $\mathcal{R}$ obtained from the fit with the kinematical power corrections, as in Fig.11

the kinematical power corrections, respespectively. The shaded area around the dashed curve shows $1 \sigma$ error bands arising from the ambiguity in extraction of $\mathcal{R}$ from the WACS data. As we discussed in Sec.5 the kinematical corrections increase the extracted value $\mathcal{R}$ and therefore the solid curve lies a bit higher. The calculation shows the qualitative trend of the data, which increase when $\varepsilon \rightarrow 1$. However our estimates are considerably smaller than the data point at large $\varepsilon$. This observation can be considered as an indication that the contribution associated with the amplitude $g_{3}$ is probably quite sizable. In our estimate we however used approximation $g_{3} \simeq 0$. If such scenario is realized then the similar contribution to $g_{1}$ must also be large in order to describe correctly the reduced cross section.

The other important issue is that the TPE amplitude $\frac{\nu}{m^{2}} \tilde{F}_{3}$ does not vanish at $\varepsilon=1$ as it has been assumed in phenomenological analysis in Ref.[66]. Notice that this behavior is closely associated with soft spectator contribution.

Taking into account the effect of the computed TPE corrections in the transverse 
polarization yields

$$
\begin{aligned}
-\sqrt{\frac{\tau(1+\varepsilon)}{2 \varepsilon}} \frac{P_{t}}{P_{l}} & =R+\frac{1}{G_{M}}\left(\frac{\nu}{m^{2}} \tilde{F}_{3}+\delta \tilde{G}_{E}-G_{E} \delta_{2 \gamma}^{\mathrm{MT}}\right) \\
& -\frac{R}{G_{M}}\left(\frac{2 \varepsilon}{1+\varepsilon} \frac{\nu}{m^{2}} \tilde{F}_{3}+\delta \tilde{G}_{M}-G_{M} \delta_{2 \gamma}^{\mathrm{MT}}\right) .
\end{aligned}
$$

In the limit $\varepsilon \rightarrow 1$, the TPE corrections vanish:

$$
-\sqrt{\tau} \frac{P_{t}}{P_{l}}(\varepsilon=1)=R \equiv \frac{G_{E}}{G_{M}} .
$$

Taking into account assumption (7.24) and the fact that the second term in Eq.(7.33) is proportional to $R$ we obtain the following estimate for the TPE correction

$$
-\sqrt{\frac{\tau(1+\varepsilon)}{2 \varepsilon}} \frac{P_{t}}{P_{l}}=R+\frac{\alpha}{\pi} \times \mathcal{O}(R) .
$$

This allows one to conclude that the expected correction can be smaller than $1 \%$. The results of GEp- $2 \gamma$ collaboration [6] are in agreement with this conclusion. Within their error bars of order $1 \%$ this experiment does not see any systematic TPE effect on this observable.

For illustration in Fig.18 we show the effect provided by the known TPE contribution on the rhs of Eq.(7.33) $\frac{2 \varepsilon}{1+\varepsilon} \frac{\nu}{m^{2}} \tilde{F}_{3}+\delta \tilde{G}_{M}-G_{M} \delta_{2 \gamma}^{\mathrm{MT}}$. The solid line is an $\varepsilon$-independent fit of $R$. The dotted line shows the effect of the computed part of the TPE correction, the curve is restricted by region $\varepsilon>0.6\left(|u|>1.83 \mathrm{GeV}^{2}\right)$ We can clearly see that this effect is smaller than the experimental error bars.

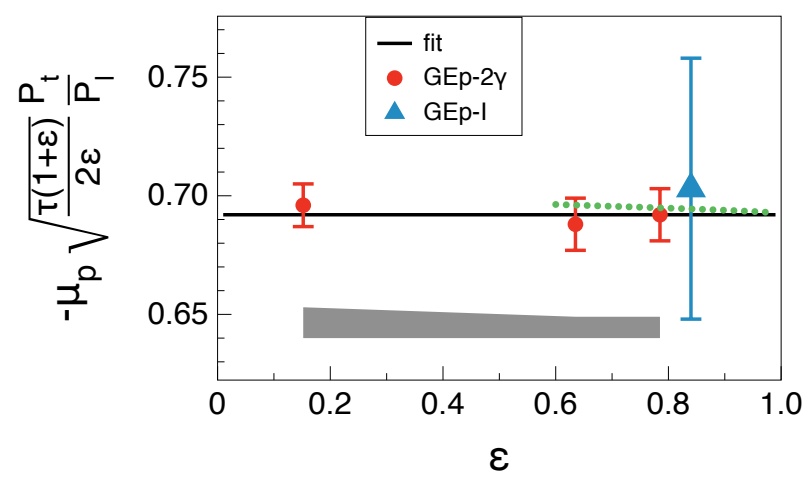

Figure 18. The ratio $-\mu_{p} \sqrt{\tau(1+\varepsilon) / 2 \varepsilon} P_{t} / P_{l}$ as function of $\varepsilon$ for $Q^{2}=2.5 \mathrm{GeV}^{2}$. The data are from GEp-I (blue triangle) [1,2], and GEp- $2 \gamma$ (red circles) [6]. The solid line is an $\varepsilon$-independent fit, the dotted (green) line shows the effect of the known part of the TPE-correction.

\section{Conclusions}

In conclusion, we have discussed the calculation of the TPE amplitudes using the QCD factorization approach in the region where all Mandelstam variables are large (wideangle scattering) $s \sim-t \sim-u \gg \Lambda^{2}$. The leading power behavior of these amplitudes are 
described by the sum of the hard and soft spectator scattering contributions. The hard spectator contribution at leading order accuracy in $\alpha_{s}$ was studied before in $[32,33]$. The soft spectator contribution was estimated before using the handbag approach in [39]. The aim of this work is to develop a more systematic description of the latter contribution in order to reduce the model dependent assumptions.

In our work we used the SCET framework and suggested a more rigorous factorization formula for the description of the soft spectator contribution. We carried out the leading power analysis in the hard scale $1 / Q^{2}$ for the TPE amplitudes and obtained that the soft spectator contributions to the TPE amplitudes can be presented as a sum of two terms associated with the different hard configurations of the photons.

If both photons are hard then the corresponding amplitude is described by the hard coefficient function and SCET FF denoted by $\mathcal{F}_{1}$. This function describes the soft overlap contribution between incoming and outgoing proton and depends on the hard-collinear scale of order $\Lambda Q$. Therefore this function depends only on the momentum transfer $Q$. The $\varepsilon$-dependence is completely described by the hard coefficient function and can be computed in perturbation theory.

If one of the photons is soft then the corresponding SCET amplitudes denoted by $g_{1,3}$ are different. These functions describe complicated low-energy hard-collinear and soft dynamics. In case of the soft spectator scattering the soft photon is involved in interactions with the hard-collinear and soft constituents of the proton. In this case the $\varepsilon$ dependence arises due to these long distance interactions.

At moderate values of $Q^{2}$ when the hard-collinear scale is not large $Q \Lambda \leq m^{2}$ the SCET amplitudes describe the long distance interaction and cannot be computed in pQCD. In order to estimate these quantities we used different approaches.

The contribution of the FF $\mathcal{F}_{1}$ can be estimated using the universality of its definition in the SCET. It turns out that this FF also arises in the factorization of the wide-angle Compton scattering amplitude (WACS). We considered this process at leading order in $1 / s$ and in the coupling $\alpha_{s}$ and provided the SCET factorization for the soft spectator contribution of the WACS amplitudes. We found that the soft spectator contribution of all three dominant amplitudes are described by the same SCET FF $\mathcal{F}_{1}$. This allows us to establish the specific relations between these independent amplitudes. Assuming the dominance of the soft-overlap mechanism we extracted the values of the dominant amplitudes in the region $Q^{2}=2.5-6.5 \mathrm{GeV}^{2}$ using the JLab Hall-A data for the WACS cross section. The contribution from the SCET FF $\mathcal{F}_{1}$ can be related to the physical WACS amplitude and this provides an estimate of this part of the TPE contribution.

A description of the amplitudes $g_{1,3}$ is more challenging because the corresponding configuration is very specific and it is difficult to find a process where they also enter. Moreover, the amplitude $g_{1}$ also includes the QED IR-divergent part which must cancel against inelastic terms. Therefore in order to estimate these amplitudes we assumed that the dominant contribution might be associated with the exchange of the very soft photon momentum. Such photon interacts with the total charge of the proton and cannot resolve the charges of the constituent quarks. In this case one can estimate the $g_{1,3}$ using an effective theory with nucleon degrees of freedom similar to the hadronic model which has 
been used for TPE calculations at relatively small $Q^{2}$ [12-17]. We suggested a somewhat similar calculation using for simplicity only the nucleon as intermediate state. The resulting expression for $g_{1}$ provides the correct QED IR-behavior and does not include any large logarithms which can be associated with the contribution of the hard region (in contrast to the MT calculation [10]). This point is very important because the subprocesses with the hard virtualities are described by the hard coefficient functions.

The amplitude $g_{3}$ does not have any IR-divergence and we obtained that $g_{3} \simeq 0$ in the framework of the simple hadronic model with the nucleon as intermediate state. This allows one to conclude that the dominant contribution to the amplitude $g_{3}$ may arise from the region where the soft photon virtuality is of order $\Lambda^{2}$. In this case the soft photon can still resolve the hard-collinear and soft structure of the proton arising from the soft spectator scattering. An investigation of this dynamics is definitely beyond the simple hadronic model used here. In our work we do not investigate this domain and accept as a first approximation the results of the elastic hadronic calculation.

Summarizing, we defined the two dominant TPE amplitudes in the framework of the QCD factorization approach. We found that the contribution associated with the two hard photons can be computed in terms of reasonably known nonperturbative quantities: nucleon distribution amplitudes (hard spectator contribution) and SCET FF $\mathcal{F}_{1}$ (soft spectator contribution ) which can be fixed from the WACS. The largest ambiguity arises from the asymmetrical configuration with hard and soft photons which is described in terms of the SCET amplitudes $g_{1,3}$. The latter can only be computed in the framework of low-energy models. For simplicity, we consider a scenario when these amplitudes are dominated by a very soft photon which already interacts with the hadron as a point-like particle. The computed TPE corrections have been used in a phenomenological analysis of the experimental data in the region where $|t|,|u|>2.5 \mathrm{GeV}^{2}$. We obtained that the corresponding TPE corrections are linear in $\varepsilon$ but relatively small in magnitude so that already for $Q^{2}>2.5-3 \mathrm{GeV}^{2}$ a description of the reduced cross section is not compatible anymore with the pure linear fit used for the Rosenbluth separation in the whole interval $0<\varepsilon<1$. Qualitatively this observation coincides with the hadronic model calculations which also show the similar trend for the moderate values of $Q^{2}$.

We also investigated the ratio $R_{e^{+} / e^{-}}$which is sensitive to the same combination of the TPE amplitudes as in the reduced cross section. We obtained that our predictions with $g_{3} \simeq 0$ for the $R_{e^{+} / e^{-}}$at $Q^{2}=2.5-4.1 \mathrm{GeV}^{2}$ are considerably smaller than the estimates originating from the linear fit of the reduced cross section, see e.g. [66].

However in the considered model such a small TPE effect in $\sigma_{R}$ can also be a consequence of the assumptions about the nonperturbative amplitudes $g_{1,3}$. We suppose that the used hadronic model can underestimate the contribution of these functions. The important information about the amplitude $g_{3}$ can be obtained from the polarization observable $P_{l}$. We found that the ratio $P_{l} / P_{l}^{\text {Born }}$ is dominated by the soft spectator contribution. However using $g_{3} \simeq 0$ our estimates yield a value for the ratio of $0.5-1 \%$ for $Q^{2}=2.5 \mathrm{GeV}^{2}$ and $\varepsilon>0.7$, falling short of the data. Further measurements of $P_{l} / P_{l}^{\text {Born }}$ for different values of $\varepsilon$ and $Q^{2}$ with better accuracy may help us considerably to constrain the unknown $g_{3}$ and reduce the uncertainty in our analysis. 
The small effect of the TPE corrections in the transverse polarization $P_{t}$ can be explained by the suppression of the helicity-flip amplitudes comparing to helicity conserving ones at moderate and large $Q^{2}$ values. This assumption is realized for the proton FFs in the smallness of the ratio $G_{E} / G_{M}$ and we expect that such suppression also holds for the corresponding combinations of the TPE amplitudes.

Our work shows that even for the wide-angle kinematics where $s \sim-t \sim-u \gg \Lambda^{2}$ the hard TPE contribution ( both photons are hard) can not provide the dominant effect. We expect that the relatively large contribution also arises from the region where one of the photons is soft and associated with the nonperturbative hard-collinear and soft dynamics. Such scenario is supported by the large TPE effect in the longitudinal polarization $P_{l}$ which is considerably larger than predictions obtained using only the hard TPE contribution. The other indication is the relatively small hard TPE contribution to the reduced cross section in the large $\varepsilon$ region. Further measurements of the $\varepsilon$ dependence of the reduced cross section or the ratio $R_{ \pm}$at intermediate $Q^{2}$ values will test any non-linearities due to TPE effect.

\section{Acknowledgments}

This work was supported by the Helmholtz Institute Mainz.

\section{A Tree level matching}

In order to obtain the expressions (3.15) for the FFs it is enough to consider the matrix element of the electromagnetic current. We also use

$$
p=Q \frac{\bar{n}}{2}+\frac{m}{Q} \frac{n}{2}+\mathcal{O}\left(m^{2} / Q^{2}\right), \quad p^{\prime}=\frac{m}{Q} \frac{\bar{n}}{2}+Q \frac{n}{2}+\mathcal{O}\left(m^{2} / Q^{2}\right) .
$$

Let us define

$$
\begin{aligned}
& \bar{N}\left(p^{\prime}\right)=\bar{N}\left(p^{\prime}\right) \frac{\text { 帆 }}{4}+\bar{N}\left(p^{\prime}\right) \frac{\not h \hbar}{4} \equiv \bar{N}_{+}^{\prime}+\bar{N}_{-}^{\prime}, \\
& N(p)=\frac{\not h h}{4} N(p)+\frac{\not h \hbar}{4} N(p) \equiv N_{+}+N_{-} .
\end{aligned}
$$

Then from the equations of motion for nucleon spinors one obtains

$$
N_{-} \simeq \frac{m}{Q} \frac{\not h}{2} N_{+}, \bar{N}_{-}^{\prime} \simeq \frac{m}{Q} \bar{N}_{+}^{\prime} \frac{\hbar}{2}
$$

which yields

$$
\begin{gathered}
\bar{N}\left(p^{\prime}\right) \gamma^{\mu} N(p)=\bar{N}_{+}^{\prime} \gamma_{\perp}^{\mu} N_{+} G_{M}+\frac{m}{Q}\left(\bar{n}^{\mu}+n^{\mu}\right) \bar{N}_{+}^{\prime} 1 N_{+}+\mathcal{O}\left(m^{2} / Q^{2}\right) \\
\bar{N}\left(p^{\prime}\right) 1 N(p)=\bar{N}_{+}^{\prime} 1 N_{+}+\mathcal{O}\left(m^{2} / Q^{2}\right) .
\end{gathered}
$$


Therefore

$$
\begin{aligned}
\left\langle p^{\prime}\left|J_{e . m .}^{\mu}(0)\right| p\right\rangle & =\bar{N}\left(p^{\prime}\right)\left[\gamma^{\mu} G_{M}-\frac{P^{\mu}}{m} F_{2}\right] N(p) \\
& \simeq \bar{N}_{+}^{\prime} \gamma_{\perp}^{\mu} N_{+} G_{M}-\frac{Q}{4 m}(n+\bar{n})^{\mu}\left(F_{2}-\frac{4 m^{2}}{Q^{2}} G_{M}\right) \bar{N}_{+}^{\prime} 1 N_{+} .
\end{aligned}
$$

On the other hand the leading ordder SCET expression gives

$$
\left\langle p^{\prime}\left|J_{e . m .}^{\mu}(0)\right| p\right\rangle \simeq C_{A}\left\langle p^{\prime}\left|O_{+}^{\mu}(0)\right| p\right\rangle_{\mathrm{SCET}}=\bar{N}\left(p^{\prime}\right) \frac{\not h \hbar}{4} \gamma_{\perp}^{\mu} N(p) C_{A} f_{1}(Q) .
$$

Comparing (A.7) and (A.8) we obtain

$$
G_{M} \simeq C_{A} f_{1}(Q), \quad F_{2} \simeq \bar{F}_{2}+\frac{4 m^{2}}{Q^{2}} C_{A} f_{1}(Q),
$$

where $\bar{F}_{2}$ denotes the contribution from the subleading SCET operators.

\section{B Power counting analysis of the SCET operators arising in elastic ep- scattering}

In this section we consider the analysis within SCET of the different contributions to elastic $e p$-scattering arising at the leading order. We do not pretend to provide a complete proof of the factorization theorem but our arguments can be considered as a first step in this direction.

Following the standard approach, we use a generic small parameter $\lambda \sim \sqrt{\Lambda / Q}$. We classify the different regions following the terminology suggested in Refs. [46, 47]: hard $p_{h} \sim Q(1,1,1)^{9}, \quad$ semi-hard $p_{\text {sh }} \sim Q(\lambda, \lambda, \lambda)$, hard-collinear $p_{h c} \sim Q\left(1, \lambda, \lambda^{2}\right)$ or $p_{h c}^{\prime} \sim Q\left(\lambda^{2}, \lambda, 1\right)$, collinear $p_{c} \sim Q\left(1, \lambda^{2}, \lambda^{4}\right)$ or $p_{c}^{\prime} \sim Q\left(\lambda^{4}, \lambda^{2}, 1\right)$ and soft $p_{s} \sim$ $Q\left(\lambda^{2}, \lambda^{2}, \lambda^{2}\right)$. For the lepton momenta $k$ and $k^{\prime}$ we assume the similar scaling behavior but associated with the vectors $\bar{v}$ and $v$, respectively.

This implies the following power counting for the SCET QCD fields

$$
\begin{aligned}
\text { hard-collinear sector: } & \xi_{n}^{h c} \sim \lambda, \bar{n} \cdot A_{h c}^{(n)} \sim 1, A_{\perp h c}^{(n)} \sim \lambda, n \cdot A_{h c}^{(n)} \sim \lambda^{2}, \\
\text { collinear sector: } & \xi_{n}^{c} \sim \lambda^{2}, \bar{n} \cdot A_{c}^{(n)} \sim 1, A_{\perp c}^{(n)} \sim \lambda^{2}, n \cdot A_{c}^{(n)} \sim \lambda^{4}, \\
\text { soft sector: } & A_{s}^{\mu} \sim \lambda^{2}, \quad q \sim \lambda^{3} .
\end{aligned}
$$

Similar counting rules are implied for the QED collinear and soft degrees of freedom. In addition to the field relations, we also need the counting of the energetic (collinear) hadronic state. It reads

$$
\left|p_{c}\right\rangle \sim \lambda^{-2}
$$

which follows from the conventional normalization.

We first discuss the power counting of the hard-spectator contribution. The matching for the leading order contributions involves the six quark operator constructed only from

\footnotetext{
${ }^{9}$ Here we imply that the light-cone momentum components are given as $\left(n \cdot p, p_{\perp}, \bar{n} \cdot p\right)$
} 
the collinear fields $\xi_{n}, \xi_{\bar{n}}$ and Wilson lines with longitudinal collinear gluons $\bar{n} \cdot A^{(n)}$ and $n \cdot A^{(\bar{n})}$ respectively. It is the product of two twist-3 3-quark operators which define the leading twist nucleon DA (7.5). When computing the hard-spectator contribution one uses

$$
\begin{aligned}
& \mathrm{T} \exp \left[i \mathcal{L}_{\mathrm{QCD}}+i \mathcal{L}_{\mathrm{QED}}\right] \simeq \\
& \mathrm{T}\left\{\bar{\zeta}_{v} \gamma^{\mu} \zeta_{\bar{v}} \bar{\chi}_{n} \bar{\chi}_{n} \bar{\chi}_{n} * \tilde{H}^{\mu} * \chi_{\bar{n}} \chi_{\bar{n}} \chi_{\bar{n}} \exp \left[i \mathcal{L}_{\mathrm{SCET}}^{(n)}+i \mathcal{L}_{\mathrm{SCET}}^{(\bar{n})}\right]\right\}
\end{aligned}
$$

where $\chi_{n, \bar{n}}$ is defined in (3.4), $\tilde{H}^{\mu}$ denotes the leading order hard-coefficient function in position space, and where the asterisks denote the collinear convolutions. Eq. (B.2) then results in the power-counting for the hard-spectator amplitude :

$$
A_{e p}^{(h)} \sim\left\langle k^{\prime}\left|\bar{\zeta}_{v} \gamma^{\mu} \zeta_{\bar{v}}\right| k\right\rangle\left\langle p^{\prime}\left|\bar{\chi}_{n} \bar{\chi}_{n} \bar{\chi}_{n}\right| 0\right\rangle * H^{\mu} *\left\langle 0\left|\chi_{\bar{n}} \chi_{\bar{n}} \chi_{\bar{n}}\right| p\right\rangle \sim \lambda^{8}
$$

Thus the asymptotic behavior of the TPE amplitudes coincides with the scaling behavior of the nucleon Dirac FF $F_{1}$, see e.g. [31].

However the hard-spectator factorization is not complete because there is a softspectator contribution with the same scaling behavior. Moreover due to the soft-collinear overlap the definition of the hard and soft contributions depends on the rapidity regularization. Therefore in order to be consistent we will take into account all possible soft spectator configurations which provide the same power $\sim \lambda^{8}$ when passing from the SCET-I to the SCET-II framework. For that purpose we need to determine the $\lambda$ suppression factors which arise when the hard-collinear fields convert into soft and collinear fields through time-ordered products.

At a first step, we define the set of the relevant SCET-I operators. We will construct them using gauge invariant combinations of quark and gluon fields which for simplicity we will call hard-collinear or collinear jets, where the quark jet was defined in Eq.(3.4). The analogous gluon jet can be introduced as

$$
\mathcal{A}_{\mu}^{(n)} \equiv\left[W_{n}^{\dagger} D_{\perp \mu} W_{n}\right]
$$

where the derivative only acts inside the brackets. We also assume that passing from SCET-I to SCET-II we substitute $\xi \rightarrow \xi_{c}+\xi_{h c}$ and integrate out $\xi_{h c}$. This implies that the relevant SCET-I operators can be built from the collinear and hard-collinear modes. The SCET-I operator is relevant if the corresponding time-ordered product provides the overlap with the leading hard-spectator contribution in Eq.(B.5), i.e. after contraction of all hard-collinear fields the resulting SCET-II operators scale with $\lambda^{12}$ and have following structure

$$
\left(\bar{\chi}_{n} \bar{\chi}_{n} \bar{\chi}_{n}\right)(q \bar{q}[\ldots])\left(\chi_{\bar{n}} \chi_{\bar{n}} \chi_{\bar{n}}\right)
$$

where the 3-quark collinear operators are the same as in Eq.(B.5) and the ellipses denote the additional soft fields. From the structure of Eq.(B.8) it follows that the collinear gluon fields like $\mathcal{A}_{c \perp}, n \cdot \mathcal{A}_{c}^{n}$ and $\bar{n} \cdot \mathcal{A}_{c}^{\bar{n}}$ can not appear in the SCET-I operators otherwise one obtains the operators with the subleading collinear structures. Keeping this in mind we 
define the following set of the SCET-I operators (we do not specify the Dirac and color indices for simplicity). For the 2-jet operators we obtain

$$
\left\{\bar{\chi}_{n}^{h c} \gamma_{\perp} \chi_{\bar{n}}^{h c}, \bar{\chi}_{\bar{n}}^{h c} \gamma_{\perp} \chi_{n}^{h c}\right\} \sim \mathcal{O}\left(\lambda^{2}\right),
$$

For the 3 -jet operators we find (all gluon fields are hard-collinear)

$$
\begin{gathered}
\left\{\bar{\chi}_{n}^{h c} \gamma_{\perp} \mathcal{A}_{\perp}^{(n)} \chi_{\bar{n}}^{h c}, \bar{\chi}_{n}^{h c} \gamma_{\perp} \mathcal{A}_{\perp}^{(\bar{n})} \chi_{\bar{n}}^{h c},(n \leftrightarrow \bar{n})\right\} \sim \mathcal{O}\left(\lambda^{3}\right) \\
\left\{\bar{\chi}_{n}^{c} \gamma_{\perp} \mathcal{A}_{\perp}^{(n)} \chi_{\bar{n}}^{h c}, \bar{\chi}_{n}^{h c} \gamma_{\perp} \mathcal{A}_{\perp}^{(\bar{n})} \chi_{\bar{n}}^{c}, \bar{\chi}_{n}^{h c} \gamma_{\perp}\left(n \cdot \mathcal{A}^{(n)}\right) \chi_{\bar{n}}^{h c}, \bar{\chi}_{n}^{h c} \gamma_{\perp}\left(\bar{n} \cdot \mathcal{A}^{(\bar{n})}\right) \chi_{\bar{n}}^{h c},\right. \\
\left.\bar{\chi}_{\bar{n}}^{h c} \Gamma\left(n \cdot \mathcal{A}^{(n)}\right) \chi_{\bar{n}}^{h c},(n \leftrightarrow \bar{n})\right\} \sim \mathcal{O}\left(\lambda^{4}\right), \\
\left\{\bar{\chi}_{n}^{c} \gamma_{\perp}\left(n \cdot \mathcal{A}^{(n)}\right) \chi_{\bar{n}}^{h c}, \bar{\chi}_{n}^{h c} \gamma_{\perp}\left(\bar{n} \cdot \mathcal{A}^{(\bar{n})}\right) \chi_{\bar{n}}^{c}, \bar{\chi}_{\bar{n}}^{c} \Gamma\left(n \cdot \mathcal{A}^{(n)}\right) \chi_{\bar{n}}^{h c},\right. \\
\left.\bar{\chi}_{\bar{n}}^{h c} \Gamma\left(n \cdot \mathcal{A}^{(n)}\right) \chi_{\bar{n}}^{c},(n \leftrightarrow \bar{n})\right\} \sim \mathcal{O}\left(\lambda^{5}\right),
\end{gathered}
$$

where we used notation $\Gamma$ for the appropriate Dirac structures.

The full set of 4-jet operators are split on two subsets: 4-jet quark operators and 4-jet quark-gluon operators. The 4-jet quark subset is described schematically as

$$
\left\{\left(\bar{\chi}_{n} \Gamma \bar{\chi}_{n}\right)\left(\chi_{\bar{n}} \Gamma \chi_{\bar{n}}\right),\left(\bar{\chi}_{n} \Gamma \bar{\chi}_{\bar{n}}\right)\left(\chi_{\bar{n}} \Gamma \chi_{n}\right),\left(\bar{\chi}_{\bar{n}} \Gamma \bar{\chi}_{\bar{n}}\right)\left(\chi_{n} \Gamma \chi_{n}\right)\right\} \sim \mathcal{O}\left(\lambda^{4}\right)-\mathcal{O}\left(\lambda^{6}\right),
$$

where the fields $\chi_{n, \bar{n}}$ describes collinear or hard-collinear jets. The subset of the 4 -jet quark-gluon operators are built from the two quark jets and two gluon jets. This subset is quite long and we shall not write it here explicitly. The corresponding operators scale as $\lambda^{4}$ to $\lambda^{8}$. Schematically these operators can be presented in following way

$$
\begin{aligned}
& \bar{\chi}_{n} \gamma_{\perp}\left\{\mathcal{A}_{\mu}^{(n)} \mathcal{A}_{\nu}^{(\bar{n})}, \mathcal{A}_{\mu}^{(n)} \mathcal{A}_{\nu}^{(n)}, \mathcal{A}_{\mu}^{(\bar{n})} \mathcal{A}_{\nu}^{(\bar{n})}\right\} \chi_{\bar{n}},(n \leftrightarrow \bar{n}), \\
& \bar{\chi}_{n} \Gamma\left\{\mathcal{A}_{\mu}^{(n)} \mathcal{A}_{\nu}^{(\bar{n})}, \mathcal{A}_{\mu}^{(\bar{n})} \mathcal{A}_{\nu}^{(\bar{n})}\right\} \chi_{n},(n \leftrightarrow \bar{n}),
\end{aligned}
$$

where the fields $\chi_{n}$ and $\chi_{\bar{n}}$ again represent collinear or hard-collinear quantities.

We shall not consider here the other possible higher order operators which are required for the complete proof of the factorization. For simplicity we also skip pure gluon operators which can arise beyond the leading order approximation. Such consideration is quite complicated and goes beyond the scope of this paper. We leave this task for future a work.

The tree level TPE diagrams providing the leading order coefficient functions are given in Fig.19. If only one photon is hard then the SCET factorization is reduced to the FF case and it has been discussed in Ref.[31]. Therefore we consider only the configurations with two hard photons.

Let us start the discussion with the 2-jet operators (B.9). Consider the first operator $\bar{\chi}_{n}^{h c} \gamma_{\perp} \chi_{\bar{n}}^{h c}$. The matching in this case is similar to the consideration for the nucleon FFs described in Ref.[31]. In order to reproduce the required structure in Eq.(B.8) for this case, we need the following time-ordered product

$$
\begin{aligned}
& \gamma_{\perp} \int d^{4} x_{1} \int d^{4} x_{2} \int d^{4} x_{3} \mathrm{~T}\left(\bar{\chi}_{n}^{h c}, \mathcal{L}_{\xi q}^{(1)}\left(x_{1}\right), \mathcal{L}_{\xi q}^{(1)}\left(x_{2}\right), \mathcal{L}_{\xi \xi}^{(0)}\left(x_{3}\right)\right) \\
& \quad \times \int d^{4} y_{1} \int d^{4} y_{2} \int d^{4} y_{3} \mathrm{~T}\left(\chi_{\bar{n}}^{h c}, \overline{\mathcal{L}}_{\xi q}^{(1)}\left(y_{1}\right), \overline{\mathcal{L}}_{\xi q}^{(1)}\left(y_{2}\right), \overline{\mathcal{L}}_{\xi \xi}^{(0)}\left(y_{3}\right)\right),
\end{aligned}
$$



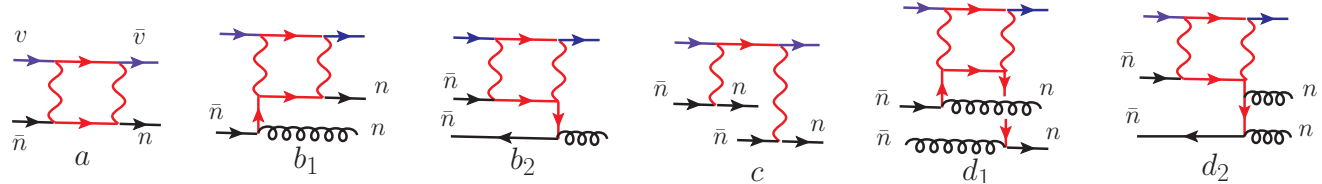

Figure 19. The examples of the TPE diagrams which can produce the different SCET-I operators discussed in Eqs.(B.9-B.14) at leading order in the QCD running coupling. We show the different operator families as different groups $(a),\left(b_{1}, b_{2}\right),(c),\left(d_{1}, d_{2}\right)$. The external collinearities are shown by labels $n$ and $\bar{n}$. The red lines specify hard virtualities, blue lines show the external leptons.

where $\mathcal{L}$ and $\overline{\mathcal{L}}$ denote the different terms of the SCET Largangian associated with the $n$ and $\bar{n}$ sectors, respectively. The explicit expressions read [44]

$$
\mathcal{L}_{\xi \xi}^{(0)}=\bar{\xi}_{n}\left(i n \cdot D+i \not D_{\perp} \frac{1}{i n \cdot D} i \not D_{\perp}\right) \frac{\not h}{2} \xi_{n}
$$

and

$$
\mathcal{L}_{\xi q}^{(1)}=\bar{\xi}_{n} i \hat{D}_{\perp} W_{n} q,
$$

which denote the leading order and next-to-leading order $\sim \mathcal{O}(\lambda)$ SCET Lagrangians respectively. For a more detailed discussion of the various aspects of matching from SCET-I to SCET-II in position space formulation of SCET we refer to Ref.[47]. In order to compute the time-ordered products in each collinear sector we need the interactions terms of the following form

$$
\begin{gathered}
\mathcal{L}_{\xi \xi, \text { int }}^{(0)}=\bar{\xi}_{n}^{c}\left(g n \cdot A_{c}^{(n)}\right) \xi_{n}^{h c}, \\
\mathcal{L}_{\xi q, \text { int }}^{(1)}=\bar{\xi}^{c} g A_{\perp h c}^{n} W_{n}^{h c} q
\end{gathered}
$$

and similarly for the $\overline{\mathcal{L}}$-contributions. The calculations in both collinear sectors are very similar, therefore we consider only one of them. One of the diagrams describing this subprocess is shown in Fig.20. The contractions of the hard-collinear fields yield:

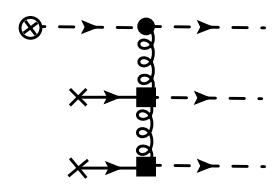

Figure 20. One of the diagrams describing the hard-collinear scattering. The crossed circle denotes the vertex of the hard-collinear operator $\left(\bar{\chi}_{n}^{h c}\right)$, the black circle describes the vertex from $\mathcal{L}_{\xi \xi}^{(0)}$, the black squares denote the vertices from $\mathcal{L}_{\xi q}^{(1)}$. The soft quark fields are shown by solid lines with crosses.

$$
\begin{gathered}
\int d^{4} x_{1}\left\langle A_{h c \perp}^{\alpha}\left(x_{1}\right) A_{h c \perp}^{\beta}\left(x_{2}\right)\right\rangle \sim \int d^{4} x_{2}\left\langle\left(\bar{n} \cdot A_{h c}\right)\left(x_{2}\right)\left(n \cdot A_{h c}\right)\left(x_{3}\right)\right\rangle \sim \lambda^{-2}, \\
\int d^{4} x_{3}\left\langle\bar{\xi}_{h c}\left(x_{1}\right) \xi_{h c}\left(x_{3}\right)\right\rangle \sim \lambda^{-2},
\end{gathered}
$$


i.e. all hard-collinear contractions cost $\lambda^{-2}$, which results from the hard-collinear propagators in momentum space. As we assume that external hard-collinear particles are matched onto collinear ones, taking account of the external collinear and soft fields we obtain:

$$
q q * J_{n} * \underbrace{\bar{\chi}_{n}^{c} \bar{\chi}_{n}^{c} \bar{\chi}_{n}^{c}}_{3 \text { coll fields }} \sim \underbrace{\lambda^{3} \lambda^{3}}_{2 \text { soft fields }} \times \underbrace{\lambda^{-2} \lambda^{-2} \lambda^{-2}}_{\text {h-coll contractions }} \times \underbrace{\bar{\chi}_{n}^{c} \bar{\chi}_{n}^{c} \bar{\chi}_{n}^{c}}_{3 \text { coll fields }} \sim \lambda^{0} \times \bar{\chi}_{n}^{c} \bar{\chi}_{n}^{c} \bar{\chi}_{n}^{c},
$$

i.e. $J_{n} \sim \lambda^{-6}$. The same counting is valid for the second jet function $J_{\bar{n}}$. The total contribution in SCET-II now reads

$$
A_{e p}^{(s)} \sim \underbrace{\left\langle k^{\prime}\left|\bar{\zeta}_{v}^{c} \gamma^{\mu} \zeta_{\bar{v}}^{c}\right| k\right\rangle}_{\lambda^{0}} \underbrace{\left\langle p^{\prime}\left|\bar{\chi}_{n}^{c} \bar{\chi}_{n}^{c} \bar{\chi}_{n}^{c}\right| 0\right\rangle}_{\lambda^{4}} * \underbrace{J_{n}}_{\lambda^{-6}} * \underbrace{\langle q q \bar{q} \bar{q}\rangle}_{\lambda^{12}} * \underbrace{J_{\bar{n}}}_{\lambda^{-6}} * \underbrace{\left\langle 0\left|\chi_{\bar{n}}^{c} \chi_{\bar{n}}^{c} \chi_{\bar{n}}^{c}\right| p\right\rangle}_{\lambda^{4}} \sim \lambda^{8} .
$$

We obtain the same power behavior for $A_{e p}^{(s)}$ as in the case of the hard sector mechanism in (B.6). The hard-collinear jet functions for this case and the soft-collinear overlap were studied in more detail in Ref. [37].

We next consider the second 2-jet operator $\bar{\chi}_{\bar{n}}^{h c} \gamma_{\perp} \chi_{n}^{h c}$ in Eq.(B.9). The difference with the previous case is that we have hard-collinear antiquarks and therefore in order to match the structure of Eq.(B.8) we have to convert antiquarks to quarks. Such a transformation in SCET-I can only be performed through two soft-collinear interactions as shown in Fig. 21. In this case the conversion of each hard-collinear antiquark costs at least one power of $\lambda$

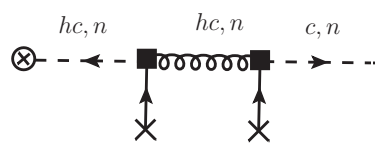

Figure 21. Quark-antiquark transition in SCET. The collinear and hard-collinear lines are shown by the labels. Notation for the vertices are the same as in Fig.20

comparing to the hard-collinear quark case. Therefore the contribution of the "antiquark" operator $\bar{\chi}_{\bar{n}}^{h c} \gamma_{\perp} \chi_{n}^{h c}$ is suppressed by a factor $\lambda^{2}$ comparing to the quark operator $\bar{\chi}_{n}^{h c} \gamma_{\perp} \chi_{\bar{n}}^{h c}$. Therefore this operator can not provide the overlap with the hard-spectator contribution (B.5). Nevertheless the contribution of the 2-jet "antiquark" operator will be suppressed only due to the hard-collinear dynamics and therefore we suppose that if the hard-collinear scale is not large $\mu_{h c} \lesssim m$ this contribution can provide sizable effects. Therefore we include it into our consideration of the soft spectator contribution. But the other higher order quark-gluon operators with the antiquarks which have structure like $\bar{\chi}_{\bar{n}} \ldots \chi_{n}$ or $\bar{\chi}_{\bar{n}} \ldots \chi_{\bar{n}}$ and $\bar{\chi}_{n} \ldots \chi_{n}$ can be discarded because they will be suppressed also at the level of the hard factorization.

Neglecting the hard-collinear antiquarks we considerably reduce the set of the 3 -jet operators which now reads

$$
\begin{aligned}
& \bar{\chi}_{n}^{h c} \gamma_{\perp}\left\{\mathcal{A}_{\perp}^{(n)}, \mathcal{A}_{\perp}^{(\bar{n})}\right\} \chi_{\bar{n}}^{h c} \sim \mathcal{O}\left(\lambda^{3}\right), \\
& \bar{\chi}_{n}^{c} \gamma_{\perp} \mathcal{A}_{\perp}^{(n)} \chi_{\bar{n}}^{h c}, \bar{\chi}_{n}^{h c} \gamma_{\perp} \mathcal{A}_{\perp}^{(\bar{n})} \chi_{\bar{n}}^{c}, \bar{\chi}_{n}^{h c} \gamma_{\perp}\left\{\left(n \cdot \mathcal{A}^{(n)}\right),\left(\bar{n} \cdot \mathcal{A}^{(\bar{n})}\right)\right\} \chi_{\bar{n}}^{h c} \sim \mathcal{O}\left(\lambda^{4}\right), \\
& \left\{\bar{\chi}_{n}^{c} \gamma_{\perp}\left(n \cdot \mathcal{A}^{(n)}\right) \chi_{\bar{n}}^{h c}, \bar{\chi}_{n}^{h c} \gamma_{\perp}\left(\bar{n} \cdot \mathcal{A}^{(\bar{n})}\right) \chi_{\bar{n}}^{c},\right\} \sim \mathcal{O}\left(\lambda^{5}\right),
\end{aligned}
$$


Constructing the time-ordered product for these operators we need to consider the new 2jet operator vertex which consists of quark and gluon jets. In the case of the hard-collinear quark jet we can proceed as before in Eq.(B.15). Therefore in the case of quark-gluon vertex we need the time-product which is matched onto the soft-collinear operator with the same structure $\left(\bar{\xi}^{c} \bar{\xi}^{c} \bar{\xi}^{c}\right)(q q)$. One can consider the same T-product, for instance

$$
\mathrm{T}\left(\bar{\chi}_{n}^{h c} \mathcal{A}_{\perp}^{(n)}, \mathcal{L}_{\xi q}^{(1)}, \mathcal{L}_{\xi q}^{(1)}, \mathcal{L}_{\xi \xi}^{(0)}\right),
$$

where the integrations are not shown for simplicity. If the quark jet is collinear then the leading order Lagrangian is not relevant and to the same order we obtain

$$
\mathrm{T}\left(\bar{\chi}_{n}^{c} \mathcal{A}_{\perp}^{(n)}, \mathcal{L}_{\xi q}^{(1)}, \mathcal{L}_{\xi q}^{(1)}\right) \text { or } \mathrm{T}\left(\bar{\chi}_{n}^{c} \mathcal{A}_{\perp}^{(n)}, \mathcal{L}_{\xi q}^{(2)}, \mathcal{L}_{\xi q}^{(1)}\right)
$$

However using the SCET counting rules we obtain that all these time-ordered products are suppressed by a factor $\lambda^{2}$ comparing to the 1-jet case considered in Eq.(B.22). For the T-products in Eqs.(B.28) it follows from the observation that the number of the hardcollinear propagators (contractions) is smaller but the external configuration is the same and this produces the extra $\lambda^{2}$ factor. If we use the leading order vertex (B.29) to compute the T-product in (B.27) then we obtain three transverse fields $A_{\perp}^{(n)}$ and therefore one of them can not be contracted and must be collinear, which not match the required structure (B.8). In order to avoid this situation instead of (B.29) we can take the term with $A_{h c \perp}^{(n)}$ and a transverse derivative

$$
\mathcal{L}_{\xi \xi, \text { int }}^{(0)} \simeq \bar{\xi}_{n}^{c} g A_{h c \perp}^{(n)} \frac{1}{i n \partial} \not_{\perp} \xi_{n}^{h c}
$$

The transverse derivative in this case yields the factor $\lambda$ and the T-product scales with $\lambda^{7}$. However the real suppression is stronger. At the tree level the transverse momenta of all particles (including the hard-collinear modes) are soft $p_{\perp} \sim \Lambda$ and the transverse derivative yields $\lambda^{2}$ factor. We expect that in case of the hard-collinear loops the additional $\lambda$ factor also arises, see for instance the discussion in Ref.[47].

The consideration of the vertices with the longitudinal projection $\left(n \cdot \mathcal{A}^{(n)}\right)$ is quite similar and shows that these contributions are also suppressed by a factor $\lambda^{2}$ compared to the 1 -jet contribution in (B.22). Therefore we conclude that 3 -jet operators can not provide the operator with structure (B.8) which scales with $\lambda^{12}$. Therefore 3 -jet operators can be neglected as subleading contributions.

The similar consideration shows that the different 4-jet quark-gluon contributions are also suppressed. But this analysis is rather lengthy and we shall not consider it here. Let us discuss only the four-jet operators listed in Eq.(B.30). Neglecting the antiquark operators we obtain only one structure

$$
\left\{\left(\bar{\chi}_{n} \Gamma \bar{\chi}_{n}\right)\left(\chi_{\bar{n}} \Gamma \chi_{\bar{n}}\right)\right\}
$$

We can conclude that the minimal configuration which can match the required structure (B.8) is described by an operator with the two soft fields $q \bar{q}$ (or one soft spectator quark). 
The operators in the both collinear sectors are the same hence we consider only one of them. The required time-ordered product reads

$$
\int d^{4} x_{1} \int d^{4} x_{2} \mathrm{~T}\left\{\left(\bar{\chi}_{n}^{c} \Gamma \bar{\chi}_{n}^{h c}\right), \mathcal{L}_{\xi q}^{(1)}\left(x_{1}\right), \mathcal{L}_{\xi \xi}^{(0)}\left(x_{2}\right)\right\}
$$

where we consider the collinear and hard-collinear jets for clarity. This choice implies that we use the interaction vertices as in (B.19) and (B.29). An example of a diagram described by this T-product is shown in Fig. 22a. Taking into account that in tree level
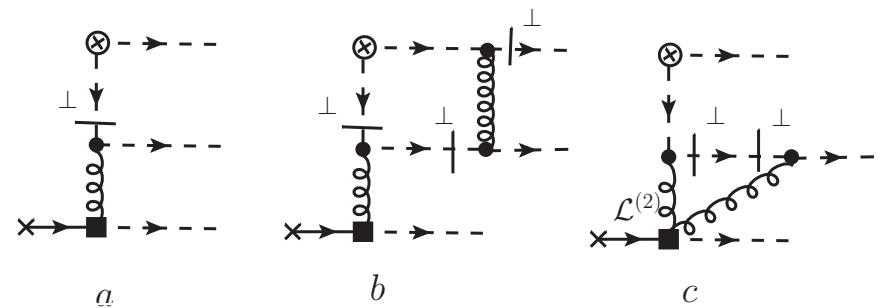

Figure 22. An example of a diagram originating in the calculation of different T-products associated with 4-jet quark operators. The notation for the lines and vertices is the same as in Fig.20. The short lines crossing the hard-collinear quark propagators denote the transverse derivatives.

diagrams $p_{\perp} \sim \Lambda$ which yields $\lambda^{2}$ we obtain that the T-product of Eq.(B.31) scales with $\lambda^{7}$. This is suppressed by a factor $\lambda$ comparing to the T-product of single quark jet in (B.22). The hard-collinear loops have the same scaling behavior because the number of transverse derivatives is always odd, see for example the diagram in Fig. 22b. Computing such loops one must have an even number of the transverse derivatives in the integrand otherwise the loop integral is trivial. Then the one derivative must always act on an external field which scales as $\lambda^{2}$ as it is shown in Fig. $22 b$ giving $\lambda^{2}$. Therefore the loop corrections also scale as $\lambda^{7}$.

An additional possibility is to insert the higher order vertex which is generated by the $\mathcal{O}\left(\lambda^{2}\right)$ Lagrangian $\mathcal{L}^{(2)}$

$$
\mathcal{L}_{\text {int }}^{(2)} \sim \xi^{c} A_{\perp}^{h c} \frac{1}{i n \cdot \partial} A_{\perp}^{h c} q
$$

In this case one can use the even number of the vertices generated by the leading order Lagrangian $\mathcal{L}_{\xi \xi}^{(0)}$. The example of the diagram generated by the corresponding time-ordered product is shown in Fig. 22c. The corresponding contribution scales again as $\lambda^{7}$. Therefore we conclude that 4-jet quark operators provide the $\mathcal{O}\left(\lambda^{2}\right)$ correction to the leading order contribution. We also take into account that these operators have larger dimension than the 2-jet operators and are therefore stronger suppressed by a hard scale after the hard factorization. Therefore we also discard these contributions.

In order to provide the complete proof that only the 2-jet operator is relevant we must also consider some other higher order operators which can potentially provide the structure of Eq.(B.8). However such consideration goes beyond the subject of this publication and we will not present it here. 


\section{Calculation of the one-loop diagrams in Fig.5}

The combination of the matrix elements which define the SCET amplitude $g_{1}^{q}$ reads

$$
\left\langle p^{\prime}\left|T\left\{O_{+}^{\mu} Y_{v}^{\dagger}(0) S_{\bar{v}}(0)\right\}\right| p\right\rangle_{\mathrm{SCET}}+\left\langle\bar{p}^{\prime}\left|T\left\{O_{+}^{\mu} Y_{v}^{\dagger}(0) S_{\bar{v}}(0)\right\}\right| \bar{p}\right\rangle_{\mathrm{SCET}}=\frac{\alpha}{\pi} g_{1}^{q}\left(z, Q, \mu_{F}\right),
$$

where the second term $\left\langle\bar{p}^{\prime}|\ldots| \bar{p}\right\rangle$ denotes the antiquark contribution. We consider only calculation of the fist matrix element on lhs (C.1). The explicit expressions for the diagrams in Fig.5 can be written as follows

$$
\begin{aligned}
D_{1 a}+D_{1 b} & =\frac{i e_{q}^{2} e^{2} \bar{\mu}^{2 \varepsilon}}{(2 \pi)^{D}} \bar{\xi}_{n} \gamma^{\mu} \xi_{\bar{n}} \int \frac{d^{D} l}{\left[l^{2}-\lambda^{2}+i \varepsilon\right]} \frac{1}{[(l \bar{n})+i \varepsilon]}\left\{\frac{(v \bar{n})}{[(v l)+i \varepsilon]}+\frac{(\bar{v} \bar{n})}{[-(\bar{v} l)+i \varepsilon]}\right\}, \\
D_{2 a}+D_{2 b} & =\frac{i e_{q}^{2} e^{2} \bar{\mu}^{2 \varepsilon}}{(2 \pi)^{D}} \bar{\xi}_{n} \gamma^{\mu} \xi_{\bar{n}} \int \frac{d^{D} l}{\left[l^{2}-\lambda^{2}+i \varepsilon\right]} \frac{1}{[(l n)+i \varepsilon]}\left\{\frac{(\bar{v} n)}{[(\bar{v} l)+i \varepsilon]}+\frac{(v \bar{n})}{[-(v l)+i \varepsilon]}\right\} .
\end{aligned}
$$

Here we assume that the renormalization scale $\bar{\mu}^{2}$ is defined in MS-scheme

$$
\bar{\mu}^{2} \equiv \mu_{F}^{2} e^{-\psi(1)-\ln 4 \pi} .
$$

One can easily see that each integral $D_{i k}$ represents the evaluation of the $T$-product of the WLs associated with the couple of the light-cone vectors: $v$ and $n, v$ and $\bar{n}$ and so on. In fact, soft photons can be decoupled from the hard-collinear quarks performing the similar redefinition of the quark fields as we did for the lepton fields in Eq.(3.26). Then one obtains that the diagrams in Fig. 5 represents the calculation of the $T$-product of the light-like WLs.

Computation of the integrals in Eqs. (C.2) and (C.3) are very similar therefore it is enough to consider only one combination. Each integral $D_{i j}$ are not well defined even in dimensional regularization and therefore one needs to introduce some additional regularization. Consider the integral in (C.2) and let us introduce the following regularized expressions

$$
D_{1 a, b}=\frac{i e_{q}^{2} e^{2} \bar{\mu}^{2 \varepsilon}}{(2 \pi)^{D}} \bar{\xi}_{n} \gamma^{\mu} \xi_{\bar{n}} J_{1 a, b}
$$

with

$$
\begin{aligned}
J_{1 a} & =\int \frac{d^{D} l}{\left[l^{2}-\lambda^{2}+i \varepsilon\right]} \frac{1}{\left[(l \bar{n})-\tau_{-}+i \varepsilon\right]} \frac{(\bar{v} \bar{n})}{\left[-(\bar{v} l)-\tau_{+}+i \varepsilon\right]}, \\
J_{1 b} & =\int \frac{d^{D} l}{\left[l^{2}-\lambda^{2}+i \varepsilon\right]} \frac{1}{\left[(l \bar{n})-\tau_{-}+i \varepsilon\right]} \frac{(v \bar{n})}{\left[(v l)-\tau_{+}+i \varepsilon\right]},
\end{aligned}
$$

where $\tau_{ \pm}$are additional regulators required in the intermediate calculations. With these regulators we can compute the integrals separately. Consider $J_{1 b}$, using light-cone vectors $\bar{n}$ and $\bar{v}$ as basic light-cone vectors:

$$
l=(l \bar{n}) \frac{v}{(v \bar{n})}+(l \bar{v}) \frac{\bar{n}}{(\bar{v} \bar{n})}+l_{\perp} \equiv l_{-} \frac{v}{(v \bar{n})}+l_{+} \frac{\bar{n}}{(\bar{v} \bar{n})}+l_{\perp},
$$


we rewrite the integrals in terms of these Sudakov variables

$$
J_{1 b}=\int d l_{+} d l_{-} d l_{\perp} \frac{1}{\left[z l_{+} l_{-}-l_{\perp}^{2}-\lambda^{2}\right]} \frac{1}{\left[l_{-}-\tau_{-}\right]} \frac{1}{\left[l_{+}-\tau_{+}\right]},
$$

where we used that

$$
d^{D} l=\frac{1}{(v \bar{n})} d l_{+} d l_{-} d^{D-2} l_{\perp}, \quad(v \bar{n})=\frac{2\left(k^{\prime} p\right)}{Q^{2}}=\frac{-u}{Q^{2}}=\frac{\bar{z}}{z} .
$$

The obtained integral can be easily computed using residues and then integrating over transverse momenta:

$$
\begin{gathered}
J_{1 b}=(-2 \pi i) \int_{0}^{\infty} d l_{-} d l_{\perp} \frac{1}{\left[z l_{-} \tau_{+}+\lambda^{2}+l_{\perp}^{2}\right]} \frac{1}{\left[l_{-}+\tau_{-}\right]} \\
=(-2 \pi i) \Gamma(\varepsilon) \int_{0}^{\infty} d l_{-} \frac{1}{\left[z l_{-} \tau_{+}+\lambda^{2}\right]^{\varepsilon}} \frac{1}{\left[l_{-}+\tau_{-}\right]} . \\
=(-2 \pi i) \frac{\Gamma(\varepsilon)}{\lambda^{2 \varepsilon}}\left\{\frac{1}{\varepsilon}-\ln \left[\frac{z \tau_{+} \tau_{-}}{\bar{z} \lambda^{2}}\right]-\psi(1+\varepsilon)+\psi(1)\right\}+\mathcal{O}\left(\tau_{+} \tau_{-}\right) .
\end{gathered}
$$

The second integral can be computed in the same way, we need only to take into account that

$$
\begin{gathered}
(\bar{v} \bar{n})=\frac{2(k p)}{Q^{2}}=\frac{s}{Q^{2}}=\frac{1}{z} . \\
J_{1 a}=2 \pi i \frac{\Gamma(\varepsilon)}{\lambda^{2 \varepsilon}}\left\{\frac{1}{\varepsilon}-\ln \left[\frac{-z \tau_{+} \tau_{-}}{\lambda^{2}}\right]-\psi(1+\varepsilon)+\psi(1)\right\}+\mathcal{O}\left(\tau_{+} \tau_{-}\right) .
\end{gathered}
$$

Therefore the sum reads

$$
\begin{gathered}
J_{1 a}+J_{1 b}=2 \pi i \frac{\Gamma(\varepsilon)}{\lambda^{2 \varepsilon}}\left\{\ln \left[\frac{z \tau_{+} \tau_{-}}{\bar{z} \lambda^{2}}\right]-\ln \left[\frac{z \tau_{+} \tau_{-}}{\bar{z} \lambda^{2}}\right]\right\} \\
=-2 \pi i \frac{\Gamma(\varepsilon)}{\lambda^{2 \varepsilon}} \ln \bar{z}
\end{gathered}
$$

where we keep only the real part. Then

$$
D_{1 a}+D_{1 b}=\frac{e_{q}^{2} e^{2}}{8 \pi^{2}} \bar{\xi}_{n} \gamma^{\mu} \xi_{\bar{n}}\left\{\frac{1}{\varepsilon} \ln \bar{z}+\ln \bar{z} \ln \frac{\mu_{F}^{2}}{\lambda^{2}}\right\} .
$$

The second sum can be computed similarly and the sum of the all terms reads

$$
D_{1 a}+D_{1 b}+D_{2 a}+D_{2 b}=\frac{\alpha}{\pi} \bar{\xi}_{n} \gamma^{\mu} \xi_{\bar{n}} e_{q}^{2}\left\{\frac{1}{\varepsilon} \ln \bar{z}+\ln \bar{z} \ln \frac{\mu_{F}^{2}}{\lambda^{2}}\right\} .
$$

The antiquark can be computed in the same way. The UV-pole $1 / \varepsilon$ is removed by the renormalization and the final expression for the SCET ampltude $g_{1}^{q}$ reads

$$
g_{1}^{q}\left(z, Q, \mu_{F}\right)=\ln \bar{z} \ln \frac{\mu_{F}^{2}}{\lambda^{2}} \mathcal{F}_{1}^{q} .
$$




\section{Cancellation of the contribution from the hard-collinear regions in the box diagrams}

In order to see the cancellation of contributions with the hard-collinear photons we suggest to compute the diagrams in Fig.4 using the strategy of regions [71, 72]. Using the IRregularization with off-shell external momenta we obtain

$$
D_{1,2}=-i \frac{e^{4} e_{q}^{2}}{(2 \pi)^{D}} J_{1,2},
$$

where subscript 1,2 denotes the box and the crossed box diagram in Fig.4, respectively. Corresponding integrals read

$$
\begin{aligned}
& J_{1}=\int d l \frac{\bar{u}_{\bar{v}} \gamma^{\nu}(\not k-\not l) \gamma^{\mu} u_{v}}{\left[l^{2}-2(l k)-k_{\perp}^{2}\right]} \frac{1}{\left[l^{2}\right]\left[\left(l+p-p^{\prime}\right)^{2}\right]} \frac{\bar{\xi}_{n} \gamma^{\nu}(\not l+\not p) \gamma^{\mu} \xi_{\bar{n}}}{\left[l^{2}+2(l p)-p_{\perp}^{2}\right]}, \\
& J_{2}=\int d l \frac{\bar{u}_{\bar{v}} \gamma^{\nu}\left(\not k^{\prime}+\not l\right) \gamma^{\mu} u_{v}}{\left[l^{2}+2\left(l k^{\prime}\right)-k_{\perp}^{2}\right]} \frac{1}{\left[l^{2}\right]\left[\left(p^{\prime}-p-l\right)^{2}\right]} \frac{\bar{\xi}_{n} \gamma^{\nu}(\not l+\not p) \gamma^{\mu} \xi_{\bar{n}}}{\left[l^{2}+2(l p)-p_{\perp}^{2}\right]} .
\end{aligned}
$$

where we assume that poles in the brackets [...] are always defined with $+i \varepsilon$ prescription.

In the collinear to $p$ region we obtain

$$
J_{1 c p}=\bar{u}_{\bar{v}} \gamma^{\nu} u_{v} \bar{\xi}_{n} \gamma^{\nu} \xi_{\bar{n}} \int d l \frac{2 k^{\mu}}{\left[-2(l k)-k_{\perp}^{2}\right]} \frac{1}{\left[-2 p^{\prime}(l+p)\right]}\left\{\frac{2(l+p)^{\mu}}{\left[l^{2}\right]\left[l^{2}+2(l p)-p_{\perp}^{2}\right]}\right\},
$$

and

$$
J_{2 c p}=\bar{u}_{\bar{v}} \gamma^{\nu} u_{v} \bar{\xi}_{n} \gamma^{\nu} \xi_{\bar{n}} \int d l \frac{2 k^{\prime \mu}}{\left[2\left(l k^{\prime}\right)-k_{\perp}^{2}\right]} \frac{1}{\left[-2 p^{\prime}(l+p)\right]}\left\{\frac{2(l+p)^{\mu}}{\left[l^{2}\right]\left[l^{2}+2(l p)-p_{\perp}^{2}\right]}\right\} .
$$

Therefore

$$
\begin{aligned}
J_{1 c p}+J_{2 c p} & =\bar{u}_{\bar{v}} \gamma^{\nu} u_{v} \bar{\xi}_{n} \gamma^{\nu} \xi_{\bar{n}} \int d l \frac{1}{\left[-2 p^{\prime}(l+p)\right]}\left\{\frac{2(l+p)^{\mu}}{\left[l^{2}\right]\left[l^{2}+2(l p)-p_{\perp}^{2}\right]}\right\} \\
& \times\left(\frac{2 k^{\mu}}{\left[-2(l k)-k_{\perp}^{2}\right]}+\frac{2 k^{\prime \mu}}{\left[2\left(l k^{\prime}\right)-k_{\perp}^{2}\right]}\right)
\end{aligned}
$$

Consider expression in (...).

$$
(\ldots) \simeq \frac{k_{-} n^{\mu}}{\left[-l_{+} k_{-}-k_{\perp}^{2}\right]}+\frac{k_{-}^{\prime} n^{\mu}}{\left[l_{+} k_{-}^{\prime}-k_{\perp}^{2}\right]}=\frac{n^{\mu}}{\left[-l_{+}-k_{\perp}^{2} / k_{-}\right]}+\frac{n^{\mu}}{\left[l_{+}-k_{\perp}^{2} / k_{-}^{\prime}\right]} .
$$

Notice that the IR-regulator $k_{\perp}^{2}$ introduces the small difference. Consider the soft limit $l_{\mu} \sim \Lambda$ in the collinear integrals in Eqs.(D.6). We obtain

$$
\begin{aligned}
\left(J_{1 c p}+J_{2 c p}\right)_{s}=\bar{u}_{\bar{v}} \gamma^{\nu} u_{v} \bar{\xi}_{n} \gamma^{\nu} \xi_{\bar{n}} & \int d l \frac{1}{q^{2}}\left\{\frac{2 p^{\mu}}{\left[l^{2}\right]\left[2(l p)-p_{\perp}^{2}\right]}\right\} \\
& \times\left(\frac{n^{\mu}}{\left[-l_{+}-k_{\perp}^{2} / k_{-}\right]}+\frac{n^{\mu}}{\left[l_{+}-k_{\perp}^{2} / k_{-}^{\prime}\right]}\right) .
\end{aligned}
$$


This expression coincides with the soft limit taken in the original integrals $J_{1,2}$. Therefore in order to obtain the collinear contribution we must subtract the overlapping soft part, see e.g. [60, 61]. This yields

$$
\left(J_{1 c p}+J_{2 c p}\right)-\left(J_{1 c p}+J_{2 c p}\right)_{s}=\bar{u}_{\bar{v}} \gamma^{\nu} u_{v} \bar{\xi}_{n} \gamma^{\nu} \xi_{\bar{n}} J_{c p}
$$

with

$$
\begin{gathered}
J_{c p}=\int d l\left(\frac{1}{\left[-2 p^{\prime}(l+p)\right]} \frac{2(l+p)^{\mu}}{\left[l^{2}\right]\left[l^{2}+2(l p)-p_{\perp}^{2}\right]}-\frac{1}{q^{2}} \frac{2 p^{\mu}}{\left[l^{2}\right]\left[2(l p)-p_{\perp}^{2}\right]}\right) \\
\times\left[\frac{n^{\mu}}{\left(-l_{+}-k_{\perp}^{2} / k_{-}\right]}+\frac{n^{\mu}}{\left[l_{+}-k_{\perp}^{2} / k_{-}^{\prime}\right)}\right] .
\end{gathered}
$$

The integral $J_{c p}$ is not singular in the region of small $l_{+}$and we can put $k_{\perp}^{2}=0$ that yileds

$$
\left(\frac{n^{\mu}}{\left[-l_{+}\right]}+\frac{n^{\mu}}{\left[l_{+}\right]}\right)=-2 \pi i \delta\left(l_{+}\right) n^{\mu}
$$

Hence

$$
\begin{gathered}
J_{c p}=(-4) \pi i \int d l \frac{\delta\left(l_{+}\right)}{\left[l^{2}\right]}\left(\frac{1}{\left[-p_{-}^{\prime}\left(l_{+}+p_{+}\right)\right]} \frac{\left(l_{+}+p_{+}\right)}{\left[l^{2}+2(l p)-p_{\perp}^{2}\right]}-\frac{1}{q^{2}} \frac{p_{+}}{\left[2(l p)-p_{\perp}^{2}\right]}\right) \\
=\frac{-4 \pi i}{q^{2}} p_{+} \int d l_{-} d l_{\perp} \frac{1}{\left[-l_{\perp}^{2}\right]}\left(\frac{1}{\left[-l_{\perp}^{2}+l_{-} p_{+}-p_{\perp}^{2}\right]}-\frac{1}{\left[l_{-} p_{+}-p_{\perp}^{2}\right]}\right) \\
=\frac{4 \pi i}{q^{2}} p_{+} \int d l_{-} d l_{\perp} \frac{1}{\left[-l_{\perp}^{2}+l_{-} p_{+}-p_{\perp}^{2}\right]\left[l_{-} p_{+}-p_{\perp}^{2}\right]} .
\end{gathered}
$$

This integral is trivial because the poles in $l_{-}$lie on the same complex semi-plane. Thus we demonstrated that after soft subtraction the collinear to $p$ integral is zero. The same manipulations also can be done for the other collinear regions.

\section{E Compensation of the QED IR-divergencies in the elastic cross section}

The dependence from the IR-regulator $\lambda$ in amplitudes $\delta \tilde{G}_{M, E}^{2 \gamma}$ arises only in the SCET amplitude $g_{1}$. Using Eqs.(2.20), (4.17) and (6.23) we obtain

$$
\delta \tilde{G}_{M}^{2 \gamma}=\frac{\alpha}{\pi} g_{1}\left(z, \mu_{0}\right)+\ldots=\frac{\alpha}{\pi} G_{M} \frac{\lambda^{2}}{\mu_{0}^{2}} \ln \left|\frac{\tilde{s}}{\tilde{u}}\right|+\ldots,
$$

where dots denote the $\lambda$-independent terms. Therefore for the corresponding difference we obtain

$$
\begin{aligned}
\operatorname{Re}\left[\delta \tilde{G}_{M}^{2 \gamma}-G_{M} \frac{1}{2} \delta_{2 \gamma}^{\mathrm{tpe}, \mathrm{MT}}\right] & =\frac{\alpha}{\pi} G_{M} \ln \frac{\lambda^{2}}{\mu_{0}^{2}} \ln \left|\frac{\tilde{s}}{\tilde{\tilde{u}}}\right|-\frac{\alpha}{\pi} G_{M} \ln \frac{\lambda^{2}}{\tilde{s}} \ln \left|\frac{\tilde{s}}{\tilde{u}}\right|+\ldots \\
& =\frac{\alpha}{\pi} G_{M} \ln \frac{\tilde{s}}{\mu_{0}^{2}} \ln \left|\frac{\tilde{s}}{\tilde{u}}\right|+\ldots
\end{aligned}
$$


The expressions (3.15) and (4.19) for the FF $F_{2}$ and amplitude $\delta \tilde{F}_{2}^{(s)}$ are incomplete because we neglected the contributions with the SCET subleading operators. Therefore we can consider cancellation of the IR-regulator $\lambda$ in the combination $\delta \tilde{G}_{E}-G_{E} \delta_{2 \gamma}$ in Eq.(7.25) only between the corresponding kinematical power corrections. Using Eqs.(4.19) yields

$$
\begin{aligned}
\delta \tilde{G}_{E}^{(s)} & =\delta \tilde{G}_{M}-(1+\tau) \delta \tilde{F}_{2}^{(s)} \\
& \simeq-\frac{\alpha}{\pi} \frac{1}{\tau}\left\{g_{1}\left(z, \mu_{0}\right)+C_{M}\left(z, \mu_{0}\right) \mathcal{F}_{1}+\frac{\nu}{s} C_{3}(z) \mathcal{F}_{1}\right\}-\frac{\nu}{s} C_{3}(z) \mathcal{F}_{1},
\end{aligned}
$$

Using Eqs.(2.20) and (6.23) we obtain

$$
\begin{aligned}
& \delta \tilde{G}_{E}^{(s)} \simeq-\frac{\alpha}{\pi} \frac{1}{\tau}\left\{g_{1}\left(z, \mu_{0}\right)+C_{M}\left(z, \mu_{0}\right) \mathcal{F}_{1}+\frac{\nu}{s} C_{3}(z) \mathcal{F}_{1}\right\} \\
& =-\frac{\alpha}{\pi} \frac{1}{\tau} g_{1}\left(z, \mu_{0}\right)+\ldots=-\frac{\alpha}{\pi} \frac{1}{\tau} G_{M}(Q) \ln \frac{\lambda^{2}}{\mu_{0}^{2}} \ln \left|\frac{\tilde{s}}{\tilde{u}}\right|+\ldots
\end{aligned}
$$

Then taking into account (3.15) where we also neglected the subleading SCET operators we obtain

$$
G_{E}=G_{M}-(1+\tau) F_{2} \simeq G_{M}-(1+\tau) \frac{1}{\tau} G_{M}=-\frac{1}{\tau} G_{M} .
$$

Taking into account Eq.(7.18) yileds

$$
\begin{aligned}
\operatorname{Re}\left[\delta \tilde{G}_{E}^{(s)}-\frac{\alpha}{\pi} G_{E} \frac{1}{2} \delta_{2 \gamma}^{\mathrm{tpe}, \mathrm{MT}}\right] & \simeq-\frac{\alpha}{\pi} \frac{1}{\tau} G_{M} \ln \frac{\lambda^{2}}{\mu_{0}^{2}} \ln \left|\frac{\tilde{s}}{\tilde{\tilde{u}}}\right|+\frac{\alpha}{\pi} \frac{1}{\tau} G_{M} \ln \frac{\lambda^{2}}{\tilde{s}} \ln \left|\frac{\tilde{s}}{\tilde{u}}\right|+\ldots \\
& =-\frac{\alpha}{\pi} \frac{1}{\tau} G_{M} \ln \frac{\tilde{s}}{\mu_{0}^{2}} \ln \left|\frac{\tilde{s}}{\tilde{u}}\right|+\ldots
\end{aligned}
$$

From this calculation we can clarify why we keep unexpanded the argument of the logarithm in Eq.(6.23): we need this otherwise the cancellation of the IR-mass $\lambda^{2}$ will be incomplete because the expression for $\delta_{2 \gamma}$ in Eq.(7.18) includes such power suppressed contributions.

\section{References}

[1] M. K. Jones et al. [Jefferson Lab Hall A Collaboration], Phys. Rev. Lett. 84, 1398 (2000).

[2] V. Punjabi et al., Phys. Rev. C 71, 055202 (2005) [Erratum-ibid. C 71:069902 (2005)].

[3] O. Gayou et al. [Jefferson Lab Hall A Collaboration], Phys. Rev. Lett. 88, 092301 (2002).

[4] A. J. R. Puckett, E. J. Brash, M. K. Jones, W. Luo, M. Meziane, L. Pentchev, C. F. Perdrisat and V. Punjabi et al., Phys. Rev. Lett. 104 (2010) 242301 [arXiv:1005.3419 [nucl-ex]].

[5] A. J. R. Puckett, E. J. Brash, O. Gayou, M. K. Jones, L. Pentchev, C. F. Perdrisat, V. Punjabi and K. A. Aniol et al., Phys. Rev. C 85 (2012) 045203 [arXiv:1102.5737 [nucl-ex]].

[6] M. Meziane et al. [GEp2gamma Collaboration], Phys. Rev. Lett. 106 (2011) 132501 [arXiv:1012.0339 [nucl-ex]].

[7] L. Andivahis, P. E. Bosted, A. Lung, L. M. Stuart, J. Alster, R. G. Arnold, C. C. Chang and F. S. Dietrich et al., Phys. Rev. D 50 (1994) 5491. 
[8] M. E. Christy et al. [E94110 Collaboration], Phys. Rev. C 70, 015206 (2004).

[9] I. A. Qattan et al. Phys. Rev. Lett. 94, 142301 (2005); I. A. Qattan, nucl-ex/0610006.

[10] Y. -S. Tsai, Phys. Rev. 122 (1961) 1898; L. W. Mo and Y. -S. Tsai, Rev. Mod. Phys. 41 (1969) 205.

[11] L. C. Maximon and J. A. Tjon, Phys. Rev. C 62 (2000) 054320 [nucl-th/0002058].

[12] P. G. Blunden, W. Melnitchouk and J. A. Tjon, Phys. Rev. Lett. 91 (2003) 142304 [nucl-th/0306076];

[13] P. G. Blunden, W. Melnitchouk and J. A. Tjon, Phys. Rev. C 72 (2005) 034612 [nucl-th/0506039];

[14] S. Kondratyuk, P. G. Blunden, W. Melnitchouk and J. A. Tjon, Phys. Rev. Lett. 95 (2005) 172503 [nucl-th/0506026].

[15] D. Borisyuk and A. Kobushkin, Phys. Rev. C 74 (2006) 065203 [nucl-th/0606030].

[16] D. Borisyuk and A. Kobushkin, Phys. Rev. C 78 (2008) 025208 [arXiv:0804.4128 [nucl-th]].

[17] D. Borisyuk and A. Kobushkin, Phys. Rev. C 86 (2012) 055204 [arXiv:1206.0155 [hep-ph]].

[18] C. F. Perdrisat, V. Punjabi and M. Vanderhaeghen, Prog. Part. Nucl. Phys. 59 (2007) 694 [hep-ph/0612014].

[19] C. E. Carlson and M. Vanderhaeghen, Ann. Rev. Nucl. Part. Sci. 57 (2007) 171 [hep-ph/0701272 [HEP-PH]].

[20] J. Arrington, P. G. Blunden and W. Melnitchouk, Prog. Part. Nucl. Phys. 66 (2011) 782 [arXiv:1105.0951 [nucl-th]].

[21] A. V. Efremov and A. V. Radyushkin, Theor. Math. Phys. 42 (1980) 97 [Teor. Mat. Fiz. 42 (1980) 147].

[22] G. P. Lepage and S. J. Brodsky, Phys. Lett. B 87, 359 (1979).

[23] V. L. Chernyak and A. R. Zhitnitsky, Phys. Rept. 112, 173 (1984).

[24] N. Isgur and C. H. Llewellyn Smith, Phys. Rev. Lett. 52, 1080 (1984).

[25] N. Isgur and C. H. Llewellyn Smith, Nucl. Phys. B 317, 526 (1989).

[26] N. Isgur and C. H. Llewellyn Smith, Phys. Lett. B 217, 535 (1989).

[27] B. L. Ioffe and A. V. Smilga, Nucl. Phys. B 216, 373 (1983).

[28] V. A. Nesterenko and A. V. Radyushkin, Phys. Lett. B 115, 410 (1982).

[29] V. M. Braun, A. Lenz, N. Mahnke and E. Stein, Phys. Rev. D 65, 074011 (2002).

[30] V. M. Braun, A. Lenz and M. Wittmann, Phys. Rev. D 73, 094019 (2006).

[31] N. Kivel and M. Vanderhaeghen, Phys. Rev. D 83 (2011) 093005 [arXiv:1010.5314].

[32] D. Borisyuk and A. Kobushkin, Phys. Rev. D 79 (2009) 034001 [arXiv:0811.0266 [hep-ph]].

[33] N. Kivel and M. Vanderhaeghen, Phys. Rev. Lett. 103 (2009) 092004 [arXiv:0905.0282 [hep-ph]].

[34] P. A. M. Guichon and M. Vanderhaeghen, Phys. Rev. Lett. 91 (2003) 142303

[hep-ph/0306007]. 
[35] A. Duncan and A. H. Mueller, Phys. Rev. D 21, 1636 (1980). A. Duncan and A. H. Mueller, Phys. Lett. B 90, 159 (1980).

[36] A. I. Milshtein and V. S. Fadin, Yad. Fiz. 33, 1391 (1981). A. I. Milshtein and V. S. Fadin, Yad. Fiz. 35, 1603 (1982).

[37] N. Kivel, Eur. Phys. J. A 48 (2012) 156 [arXiv:1202.4944 [hep-ph]].

[38] Y. C. Chen, A. Afanasev, S. J. Brodsky, C. E. Carlson and M. Vanderhaeghen, Phys. Rev. Lett. 93, 122301 (2004) [hep-ph/0403058].

[39] A. V. Afanasev, S. J. Brodsky, C. E. Carlson, Y. -C. Chen and M. Vanderhaeghen, Phys. Rev. D 72 (2005) 013008 [hep-ph/0502013].

[40] C. W. Bauer, S. Fleming and M. E. Luke, Phys. Rev. D 63, 014006 (2000).

[41] C. W. Bauer, S. Fleming, D. Pirjol and I. W. Stewart, Phys. Rev. D 63, 114020 (2001).

[42] C. W. Bauer and I. W. Stewart, Phys. Lett. B 516, 134 (2001).

[43] C. W. Bauer, D. Pirjol and I. W. Stewart, Phys. Rev. D 65, 054022 (2002).

[44] M. Beneke, A. P. Chapovsky, M. Diehl and T. Feldmann, Nucl. Phys. B 643, 431 (2002).

[45] M. Beneke and T. Feldmann, Phys. Lett. B 553, 267 (2003).

[46] R. J. Hill and M. Neubert, Nucl. Phys. B 657 (2003) 229 [hep-ph/0211018].

[47] M. Beneke and T. Feldmann, Nucl. Phys. B 685 (2004) 249 [hep-ph/0311335].

[48] A. V. Manohar, Phys. Rev. D 68, 114019 (2003).

[49] C. W. Bauer, S. Fleming, D. Pirjol, I. Z. Rothstein and I. W. Stewart, Phys. Rev. D 66 (2002) 014017 [hep-ph/0202088].

[50] P. Van Nieuwenhuizen, Nucl. Phys. B 28 (1971) 429.

[51] A. Danagoulian et al. [Hall A Collaboration], Phys. Rev. Lett. 98 (2007) 152001 [nucl-ex/0701068 [NUCL-EX]].

[52] D. J. Hamilton et al. [Jefferson Lab Hall A Collaboration], Phys. Rev. Lett. 94 (2005) 242001 [nucl-ex/0410001].

[53] S. J. Brodsky and G. R. Farrar, Phys. Rev. Lett. 31 (1973) 1153.

[54] V. A. Matveev, R. M. Muradian and A. N. Tavkhelidze, Lett. Nuovo Cim. 7 (1973) 719.

[55] A. V. Radyushkin, Phys. Rev. D 58 (1998) 114008 [hep-ph/9803316].

[56] M. Diehl, T. Feldmann, R. Jakob and P. Kroll, Eur. Phys. J. C 8 (1999) 409 [hep-ph/9811253].

[57] H. W. Huang, P. Kroll and T. Morii, Eur. Phys. J. C 23 (2002) 301 [Erratum-ibid. C 31 (2003) 279] [hep-ph/0110208].

[58] G. A. Miller, Phys. Rev. C 69 (2004) 052201 [nucl-th/0402092].

[59] D. Babusci, G. Giordano, A. I. L'vov, G. Matone and A. M. Nathan, Phys. Rev. C 58 (1998) 1013 [hep-ph/9803347].

[60] J. C. Collins and F. Hautmann, Phys. Lett. B 472, 129 (2000) [arXiv:hep-ph/9908467].

[61] A. V. Manohar and I. W. Stewart, Phys. Rev. D 76, 074002 (2007) [arXiv:hep-ph/0605001].

[62] T. Becher, G. Bell and M. Neubert, Phys. Lett. B 704 (2011) 276 [arXiv:1104.4108 [hep-ph]]. 
[63] J. Y. Chiu, A. Jain, D. Neill and I. Z. Rothstein, arXiv:1202.0814 [hep-ph].

[64] M. Beneke, G. Buchalla, M. Neubert and C. T. Sachrajda, Nucl. Phys. B 591 (2000) 313 [arXiv:hep-ph/0006124].

[65] M. Beneke and T. Feldmann, Nucl. Phys. B 592 (2001) 3 [arXiv:hep-ph/0008255].

[66] J. Guttmann, N. Kivel, M. Meziane and M. Vanderhaeghen, Eur. Phys. J. A 47 (2011) 77 [arXiv:1012.0564 [hep-ph]].

[67] E. J. Brash, A. Kozlov, S. Li and G. M. Huber, Phys. Rev. C 65 (2002) 051001 [hep-ex/0111038].

[68] S. Venkat, J. Arrington, G. A. Miller and X. Zhan, Phys. Rev. C 83 (2011) 015203 [arXiv:1010.3629 [nucl-th]].

[69] D. Borisyuk and A. Kobushkin, Phys. Rev. D 83 (2011) 057501 [arXiv:1012.3746 [hep-ph]].

[70] I. A. Qattan, A. Alsaad and J. Arrington, Phys. Rev. C 84 (2011) 054317 [arXiv:1109.1441 [nucl-ex]].

[71] M. Beneke and V. A. Smirnov, Nucl. Phys. B 522 (1998) 321 [arXiv:hep-ph/9711391],

[72] V. A. Smirnov, Springer Tracts Mod. Phys. 177 (2002) 1. 